\title{
Identification and characterization of ADNP as a novel heterochromatin component
}

\author{
Dissertation \\ for the award of the degree \\ "Doctor rerum naturalium" (Dr. rer. nat.) \\ Division of Mathematics and Natural Sciences \\ of the Georg-August-Universität Göttingen
}

submitted by

Kerstin Mosch

from Dresden

Göttingen 2010 
Members of the Thesis Committee:

Dr. Wolfgang Fischle ( $1^{\text {st }}$ reviewer), Research group Chromatin Biochemistry Max Planck Institute for Biophysical Chemistry, Göttingen

Prof. Dr. Herbert Jäckle (2 ${ }^{\text {nd }}$ reviewer), Department of Molecular Developmental Biology Max Planck Institute for Biophysical Chemistry, Göttingen

Prof. Dr. Matthias Dobbelstein, Department of Molecular Oncology Georg-August-University Göttingen

Prof. Dr. Sigrid Hoyer-Fender, Department of Developmental Biology Georg-August-University Göttingen

Date of the oral examination: August 11, 2010 
I affirm that the presented thesis has been written independently and with no other sources and aids than quoted.

June 30, 2010, Göttingen

Kerstin Mosch 



\section{Acknowledgements}

First and foremost, I would like to thank my mentor, Dr. Wolfgang Fischle, for his support and guidance. I am grateful for his constructive criticism, all the fruitful discussions, his creative and encouraging ideas that have always been a source of inspiration and motivation.

I am grateful to my thesis committee consisting of Prof. Dr. Hoyer-Fender, Prof. Dr. Herbert Jäckle, and Prof. Dr. Matthias Dobbelstein for helpful discussions and guidance throughout my $\mathrm{PhD}$ project.

I want to thank Dr. Henning Urlaub and his group for their excellent mass spectrometry work, especially Uwe Plessmann and Monika Raabe for mass spectrometry and Miroslav Nicolov for data analysis.

I appreciate the GGNB "Molecular Biology of Microbial, Animal and Plant Cells" program for the constant support, informative lectures, highly supportive method courses as well as inspiring retreats.

I owe Winfried Lendeckel thanks for his trials to produce bacculoviral ADNP and for his many hours of purifying histones.

I am thankful to Szabolcs Soeroes for offering me recombinant HP1 and supporting me in the generation of nucleosomal arrays.

I want to thank Kathy Gelato for reading and correcting the manuscript. 
Special thanks are dedicated to the members of the Fischle lab for their help and many moments of discussion. In particular, thanks to the "first batch" of PhD students in our group, Henriette, Martina, Nora and Sabi for their friendship, the entertaining hours outside the lab, the badminton trainings and teaching me to cycle.

Lastly, I am grateful to my family, in particular to my parents Gisela und Dietmar Mosch for their constant encouragement, their belief in me, and their continuous support. 


\section{Table of contents}

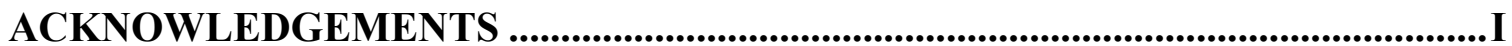

TABLE OF CONTENTS ............................................................................................

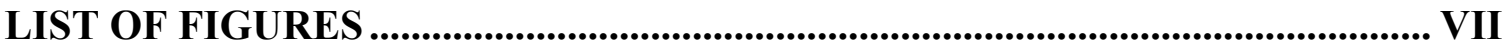

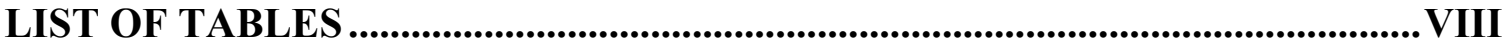

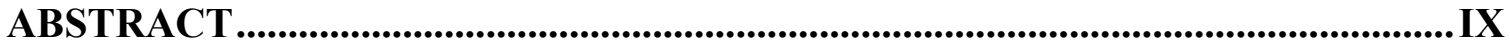

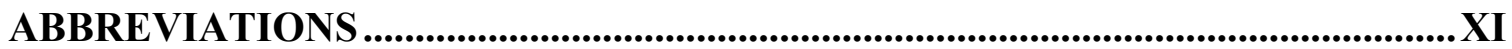

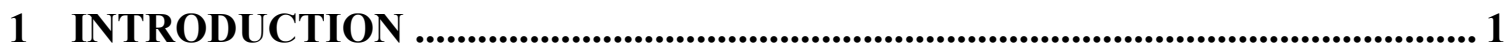

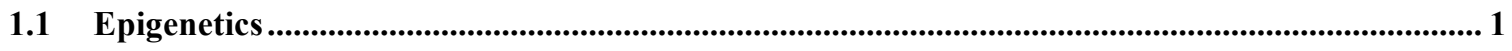

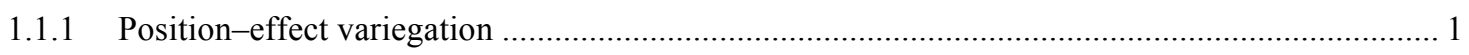

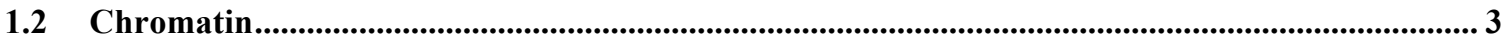

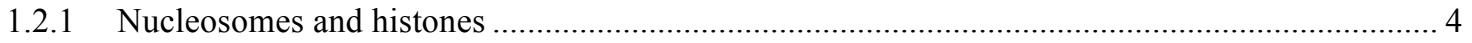

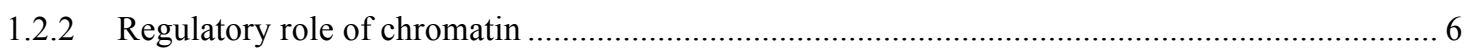

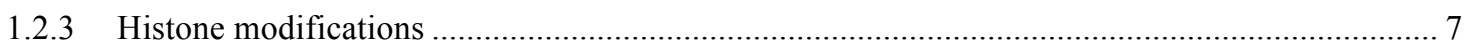

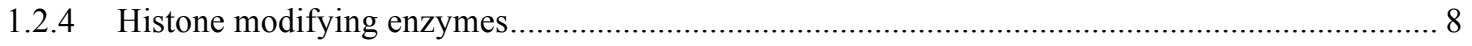

1.2.5 Distribution of histone modifications along chromosomes ......................................... 9

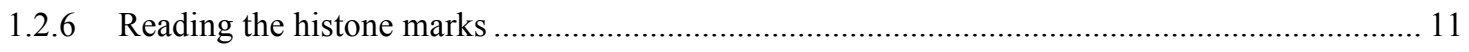

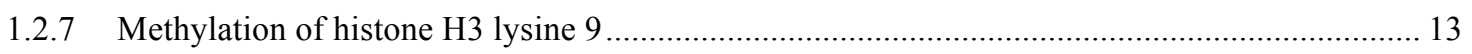

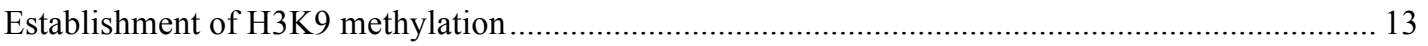

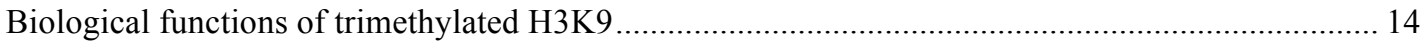

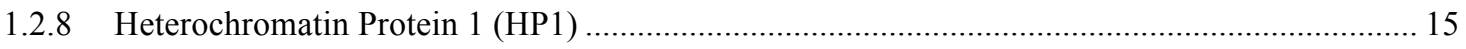

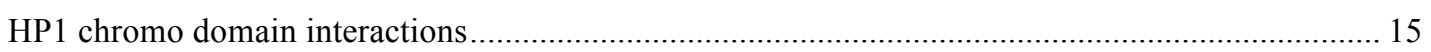

HP1 chromoshadow domain interactions ......................................................................... 16

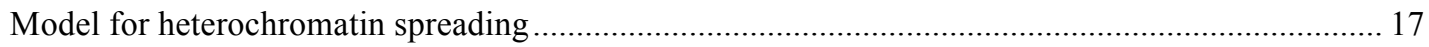


1.3 Activity-dependent neuroprotective protein (ADNP)....................................................................... 19

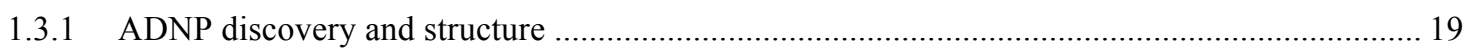

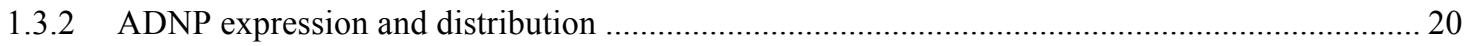

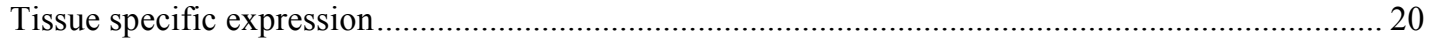

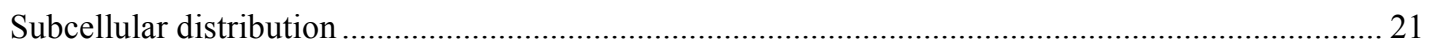

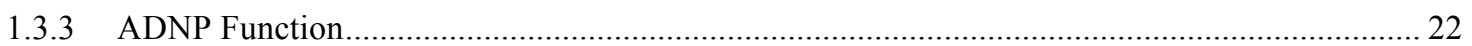

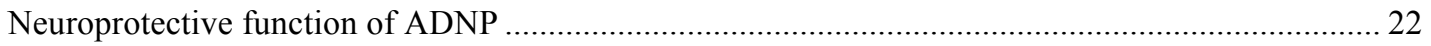

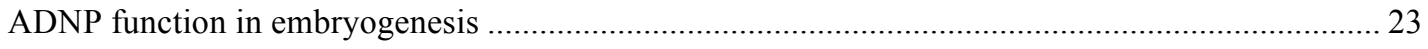

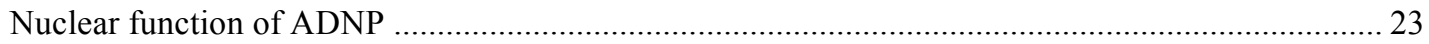

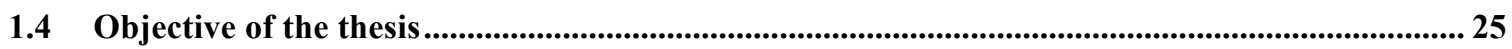

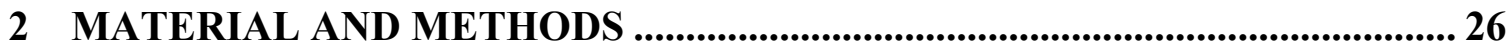

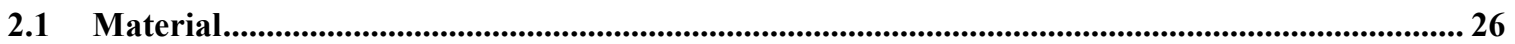

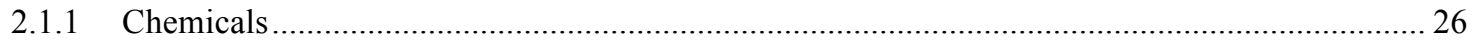

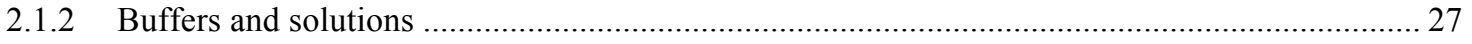

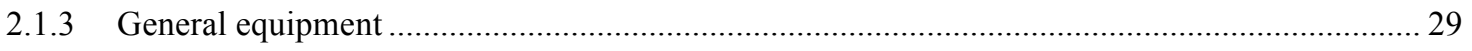

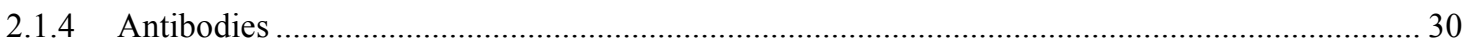

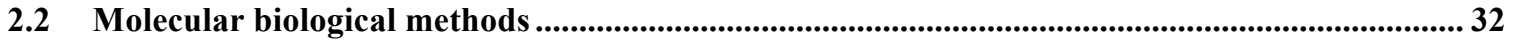

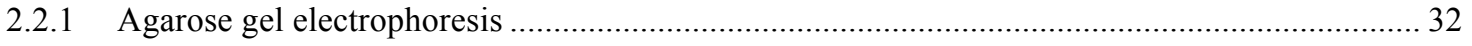

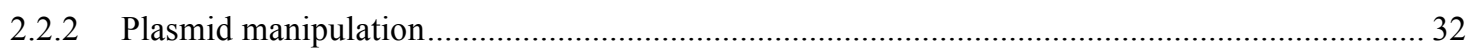

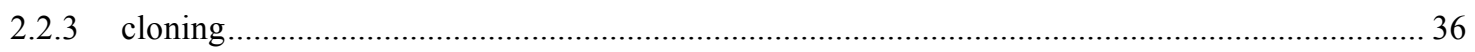

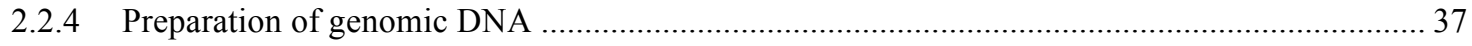

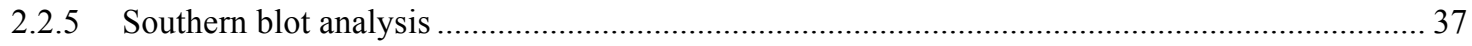

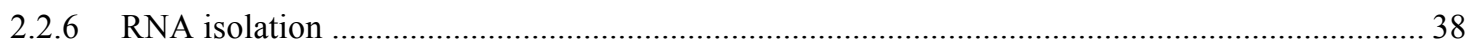

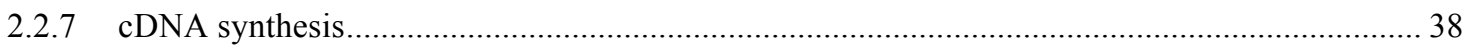

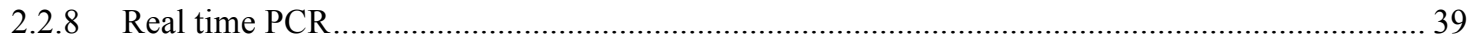

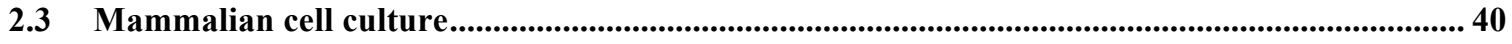

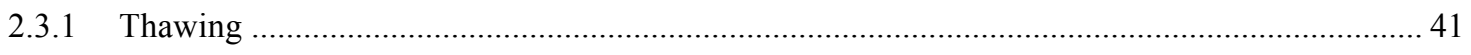

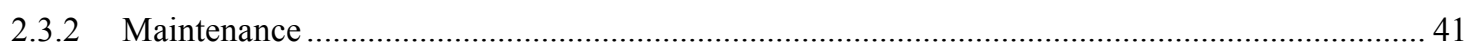

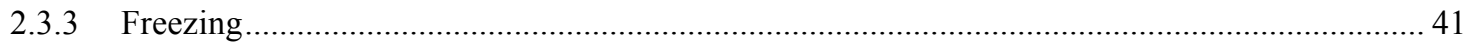

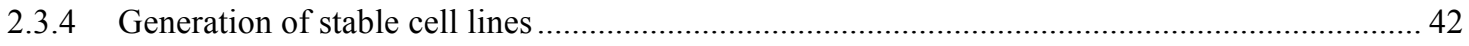

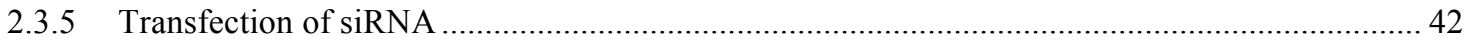

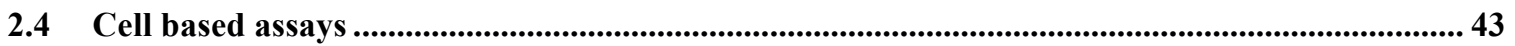

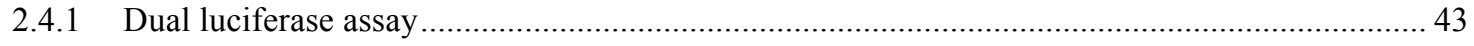




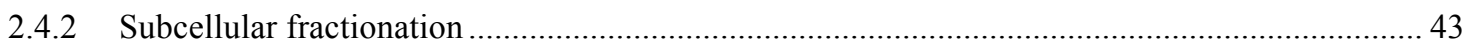

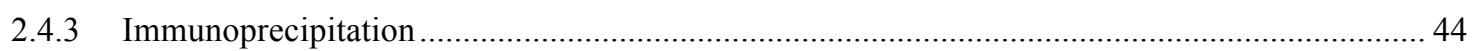

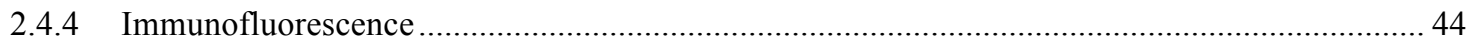

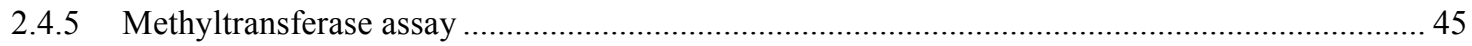

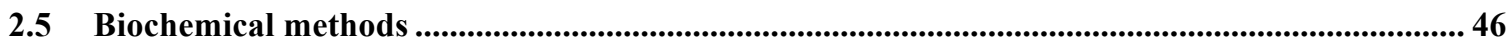

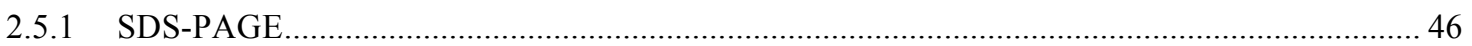

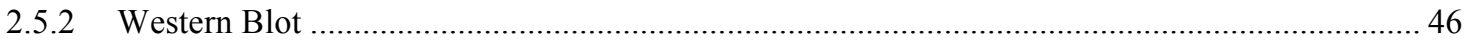

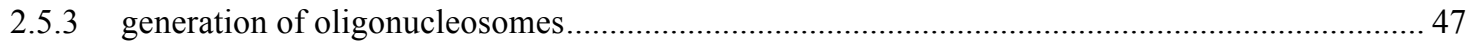

Introduction of specific histone post-translational modifications .......................................................... 47

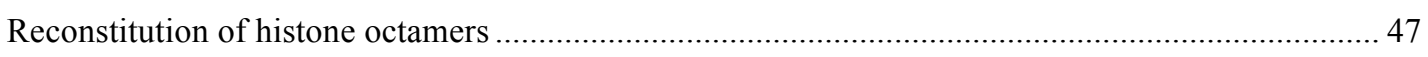

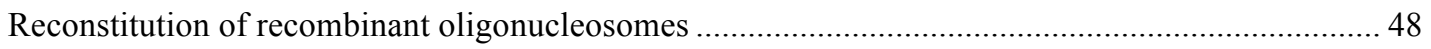

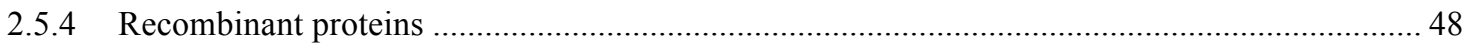

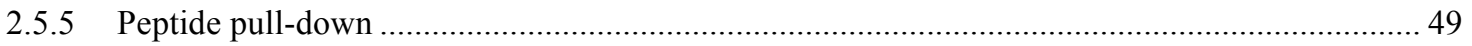

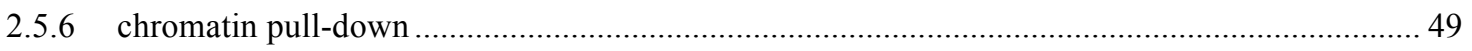

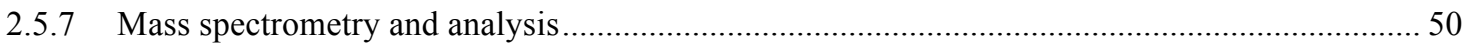

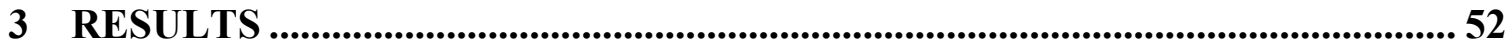

3.1 Identification of factors associated with H3K9me3 …....................................................................... 52

3.2 Verification of H3K9me3 association of ADNP ............................................................................. 55

3.2.1 ADNP associates with $\mathrm{H} 3 \mathrm{~K} 9 \mathrm{me} 3$ and pericentromeric heterochromatin.................................... 55

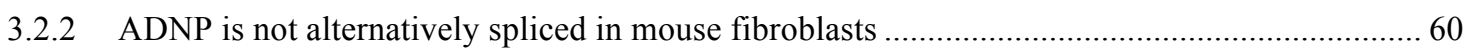

3.3 Mode of ADNP recruitment to heterochromatin ................................................................................ 62

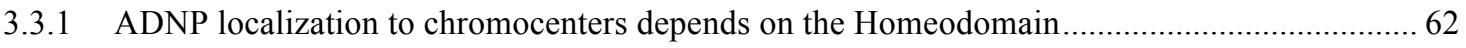

3.3.2 ADNP does not bind to H3K9me3 directly but is targeted to pericentromeric heterochromatin by HP1 64

3.3.3 ADNP localization to chromocenters mainly depends on HP1 binding to the PxVxL motif

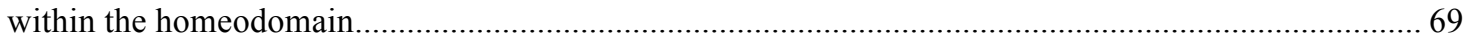

3.3.4 A possible lysine methylation in the ADNP ARKS motif is not involved in HP1 binding......... 73

3.4 ADNP function at pericentromeric heterochromatin.............................................................. 75

3.4.1 ADNP knockdown does not phenocopy the Brg1 knockout phenotype ........................................ 76

3.4.2 Knockdown of ADNP does not influence the global level of histone modifications .................. 79

3.4.3 ADNP knockdown has no influence on the localization of HP1 and repressive histone modifications.

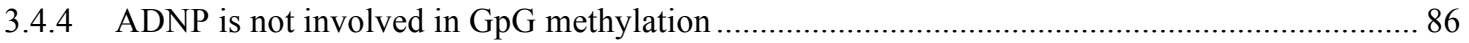




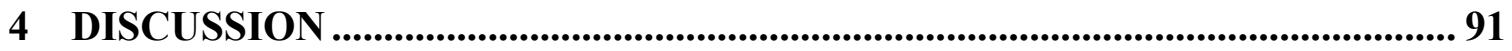

4.1 Identification of $\mathrm{H3K9me3} \mathrm{associated} \mathrm{proteins} \mathrm{..............................................................91}$

4.2 ADNP is a novel component of pericentromeric heterochromatin ...........................................92

4.3 ADNP is targeted to pericentromeric heterochromatin by HP1 .................................................94

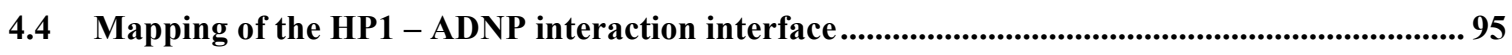

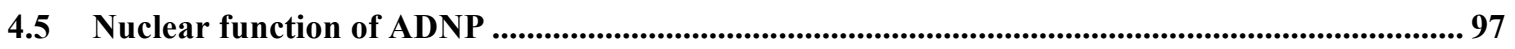

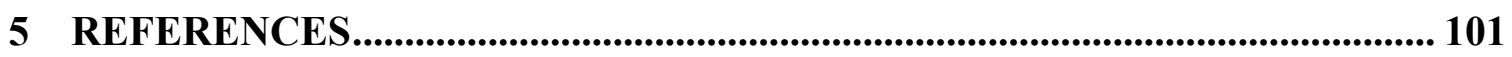

6 CURRICULUM VITAE......................................................................................... 112 


\section{List of figures}

Figure 1-1: Position effect variegation in Drosophila melanogaster ................................. 2

Figure 1-2: The organization of DNA within the cell....................................................... 3

Figure 1-3: Structure of the nucleosome core particle ................................................... 5

Figure 1-4: Histone modifications across a typical mammalian chromosome ................. 10

Figure 1-5: Molecular mechanisms of histone modifications........................................... 11

Figure 1-6: Model for the spreading of heterochromatin............................................... 18

Figure 1-7: phylogenetic tree of ADNP homologs in different species............................ 19

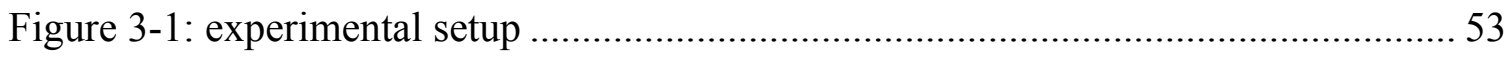

Figure 3-2: Distribution of the ratios between the heavy and light samples ..................... 54

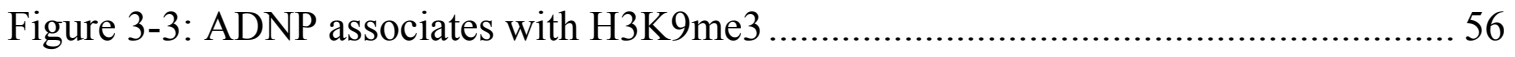

Figure 3-4 ADNP is enriched at pericentromeric heterochromatin .................................. 57

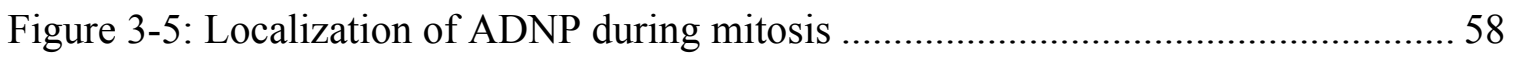

Figure 3-6: ADNP localization to pericentromeric heterochromatin depends on Suv39h 59

Figure 3-7: Only full-length ADNP mRNA is detected in mouse fibroblasts.................. 61

Figure 3-8: The homeodomain of ADNP is necessary and sufficient for localization to

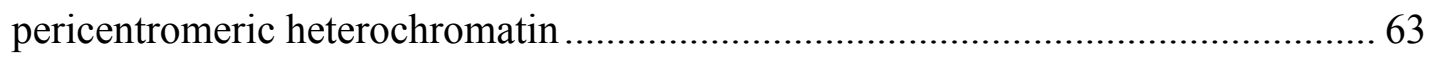

Figure 3-9 All three HP1 isoforms are able to recruit ADNP to H3K9me3 ..................... 64

Figure 3-10: ADNP localization to pericentromeric heterochromatin is HP1 dependent. 66

Figure 3-11: levels of HP1 $\gamma$, ADNP and H3K9me3 as well as H3K9me3 distribution are

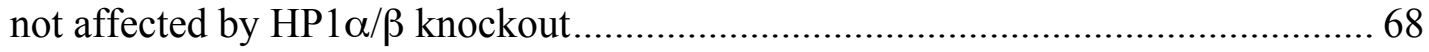

Figure 3-12: The PxVxL and ARKS motifs are involved in ADNP localization............. 70

Figure 3-13: The AKRS and PxVxL pointmutations have no influence on ADNP

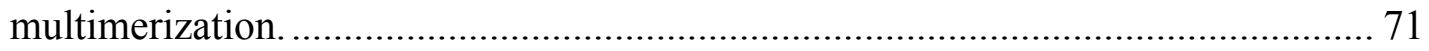

Figure 3-14: HP1 chromoshadow domain mutation strongly reduces binding of ADNP 72

Figure 3-15: The ARKS motif of ADNP is not involved in HP1 binding........................ 74

Figure 3-16: ADNP knockdown does not influence H3K9me3 localization. .................. 77

Figure 3-17: ADNP knockdown does not influence H4K20me3 distribution................... 78

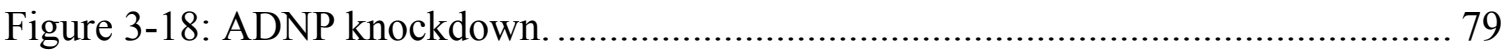


Figure 3-19: ADNP knockdown does not influence global histone modifications .......... 80

Figure 3-20: ADNP knockdown does not influence HP1 $\alpha$ localization. ........................ 82

Figure 3-21: ADNP knockdown does not influence HP1 $\beta$ localization........................... 83

Figure 3-22: ADNP knockdown does not influence HP1 $\gamma$ localization. ......................... 84

Figure 3-23: ADNP knockdown does not influence H3K27me1 distribution................. 85

Figure 3-24: ADNP knockdown has no influence on CpG-methylation......................... 86

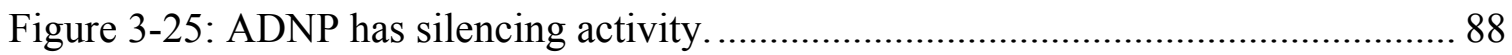

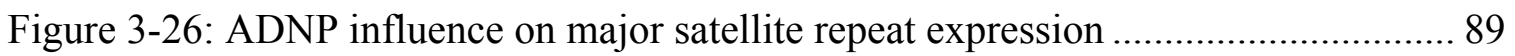

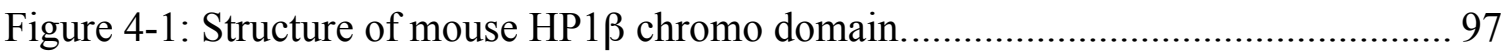

Figure 4-2: Model for ADNP recruitment and function .......................................... 100

\section{List of tables}

Table 1-1: Examples of histone marks, the responsimle enzymes and their functional

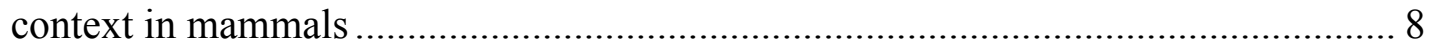

Table 1-2: domains of human ADNP (Q9H2P0) as predicted by UniProtKB ............... 20

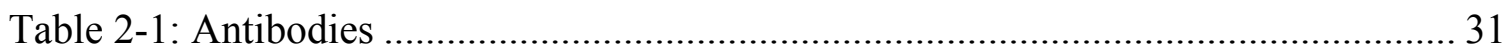

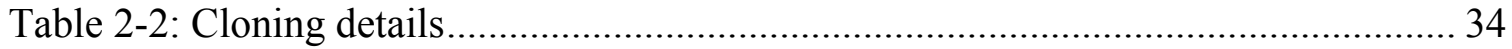

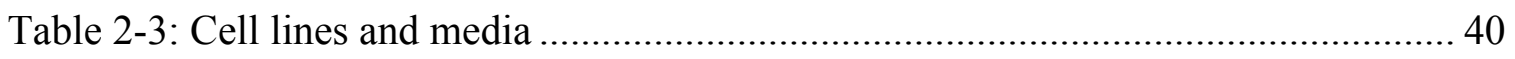

Table 2-4: Peptides used for the pulldown experiment ............................................. 49

Table 3-1: Proteins with at least 2-fold encichment in both experiments....................... 54 


\section{Abstract}

In eukaryotic cells genetic information in the form of long linear DNA fibers is stored in the cell nucleus. Time and tissue specific gene expression patterns are manifested by the combination of DNA with proteins resulting in a structure called chromatin. The basic repeating element of chromatin is the nucleosome, where DNA is wrapped around an octamer of histone proteins, interconnected by sections of linker DNA. Both DNA and histones are subject to chemical modifications, which are associated with certain chromatin states. Several factors that set or recognize such modifications have been described in the recent years.

Heterochromatin is a chromatin form that is characterized by compaction, transcriptional inactivity and late replication in S-phase. It is associated with methylation of DNA and histones. Trimethylation of histone $\mathrm{H} 3$ lysine 9 (H3K9me3) is one hallmark of heterochromatin, and is mediated by the methyltransferase Suv39 and recognized by heterochromatin protein 1 (HP1), which has three isoforms in mammalien cells. How these and other enzymes and factors establish and maintain distinct chromatin states is not yet completely understood.

For a better understanding of factors involved with heterochromatin I used stable isotope labeling by aminoacids in cell culture (SILAC) in a $\mathrm{H} 3 \mathrm{~K} 9 \mathrm{me} 3$ pull-down experiment to identify new interaction partners. Activity dependent neuroprotector (ADNP) was one such factor that had not been described in a heterochromatin context before. Association of ADNP with H3K9me3 was verified with independent experiments and the factor was further characterized.

Cell based and in vitro assays suggested that ADNP does not bind to H3K9me3 directly but is targeted to this modification by HP1. This recruitment of ADNP to H3K9me3 could be mediated by all three isoforms of HP1. Mapping of the interaction interface revealed a major contribution of the HP1 chromoshadow domain binding to a PxVxL motif within the ADNP homeodomain. Mutation of the PxVxL motif caused partial delocalization from heterochromatin. An additional mutation of an ARKS motif, which is 
also present in the ADNP homeodomain, enhanced this effect. However, involvment of a possible lysine methylation in that motif in HP1 binding was not detected.

To determine the function of ADNP I used knock-down in cells by siRNA. I analyzed whether ADNP influences typical heterochromatin features such as distribution of histone modifications as well as HP1 localization as well as DNA methylation state. However, no effect on these properties could be detected. In luciferase reporter assays ADNP displayed transcriptional silencing potential. Knock-down and overexpression experiments suggested, that ADNP is specifically involved in silencing of major satellite repeats in pericentromeric heterochromatin. Further studies are needed to address by which mechanism ADNP exerts this silencing function.

Altogether, in this work I identified ADNP as a novel component of pericentromeric heterochromatin, which is recruited by HP1 and acts in silencing of major satellite repeats. This study deepens the understanding of how the Suv39/H3K9me3/HP1 pathway impacts chromatin function. 


\section{Abbreviations}

ac

ADP

ADNP

BD

BSA

C

CD

ChIP

cDNA

CMV

CSD

DAPI

DIG

DMEM

DMSO

DNA

DNMT1

dNTPs

$\mathrm{dpt}$

DTT

E.coli

EDTA

FDR

h

H3K9me3

HAT

HDAC
Acetylation

Adenosine diphosphate

Activity dependent neuroprotector

Bromodomain

Bovine serum albumine

Celsius

Chromodomain

Chromatin immunoprecipitation

Complementary DNA

Cytomegalovirus

Chromoshadow domain

4',6-diamidino-2-phenylindole

Digoxigenin

Dulbecco's Modified Eagle's Medium

Dimethylsulfoxid

Desoxyribonucleic acid

DNA methyltransferase 1

Desoxyribonucleotide triphosphates

Days post transfection

DL-1,4-dithiothreitol

Escherichias coli

Ethylenediaminetetraacetic acid

False discovery rate

Hour

Histone H3 lysine 9 trimethylation

Histone acetyltransferase

Histone deacetylase 
HEK293

HeLaS3

HEPES

His

HMT

HP1

HRP

IF

$\mathrm{Ig}$

IP

IPTG

$\mathrm{kDa}$

me

MEF

$\min$

NAP

NURD

OD

PBS

PCR

$\mathrm{ph}$

PNV

PTM

RNA

rpm

RT

RT-PCR

SDS

SDS-PAGE

SILAC

Suv39h
Human embryonic kidney 293 cell line

Human cervix epithel carcinoma cell line

2-[4-(2-hydroxyethyl)-1-piperazinyl]-ethanesulfonic acid

Histidine

Histone methyltransferase

Heterochromatin protein 1

Horse radish peroxidase

Immunofluorescence

Immunoglobulin

Immunoprecipitation

Isopropylthio-b-D-galactoside

Kilo Dalton

Methylation

Mouse embryonic fibroblast cell line

Minutes

Neuroprotective peptide sequence in ADNP: NAPVSIPQ

Nucleosome remodeling and deacetylase complex

Optical density

Phosphate buffered saline

Polymerase chain reaction

Phosphorylation

Packed nuclear volume

Post translational modifications

Ribonucleic acid

Rounds per minute

Room temperature

Reverse transcriptase PCR

Sodium dodecyl sulfate

Sodium docecyl sulfate polyacrylamide gel electrophoresis

Stable isotope labeling with amino acids in cell culture

Suppressor of variegation 3-9 homolog 
S

TK

UAS

UTR

VIP

wt
Second

Tyrosine kinase

Upstream activator sequences

Untranslated regions

Vasoactive intestinal peptide

Wild type, original sequence without mutations 



\section{Introduction}

\subsection{Epigenetics}

The term epigenetics was coined by C. H. Waddington for the study of "causal mechanisms" by which "the genes of the genotype bring about phenotypic effects". Today an epigenetic phenomenon is defined as a change in phenotype that is heritable but does not involve DNA mutation (Allis, 2007).

In principle all tissue-specific somatic cells contain the same genetic information, which is contained within the linear DNA sequence (the genome). However, each cell type is characterized by specific gene expression patterns that need to be established and inherited throughout cell division. Transcription factors and chromatin proteins that are associated with DNA play important roles for cell type-specific gene expression. Epigenetic modifications such as DNA methylation or covalent modifications to histones as well as chromatin remodeling mediate stable activation or repression of genes in specific cell types.

\subsubsection{Position-effect variegation}

The first description of a phenomenon with an underlying epigenetic basis has been provided by the discovery of position-effect variegation (PEV) (Muller and Altenburg, 1930). Muller described mutations of the white $+\left(\mathrm{w}^{+}\right)$eye color gene of Drosophila that resulted in a variegating phenotype, with some patches of red and some patches of white facets. The mosaic phenotypes were caused by a chromosomal inversion or rearrangement in one breakpoint within the pericentromeric heterochromatin and the other adjacent to the white gene (Figure 1-1). Thus, the $\mathrm{w}^{+}$gene has been displaced from its normal euchromatic location and was placed in the vicinity of heterochromatin. This 
pattern of variegation suggested that a decision to express or repress the $\mathrm{w}^{+}$gene was made early during tissue development and was maintained in a metastable state through multiple cell divisions.

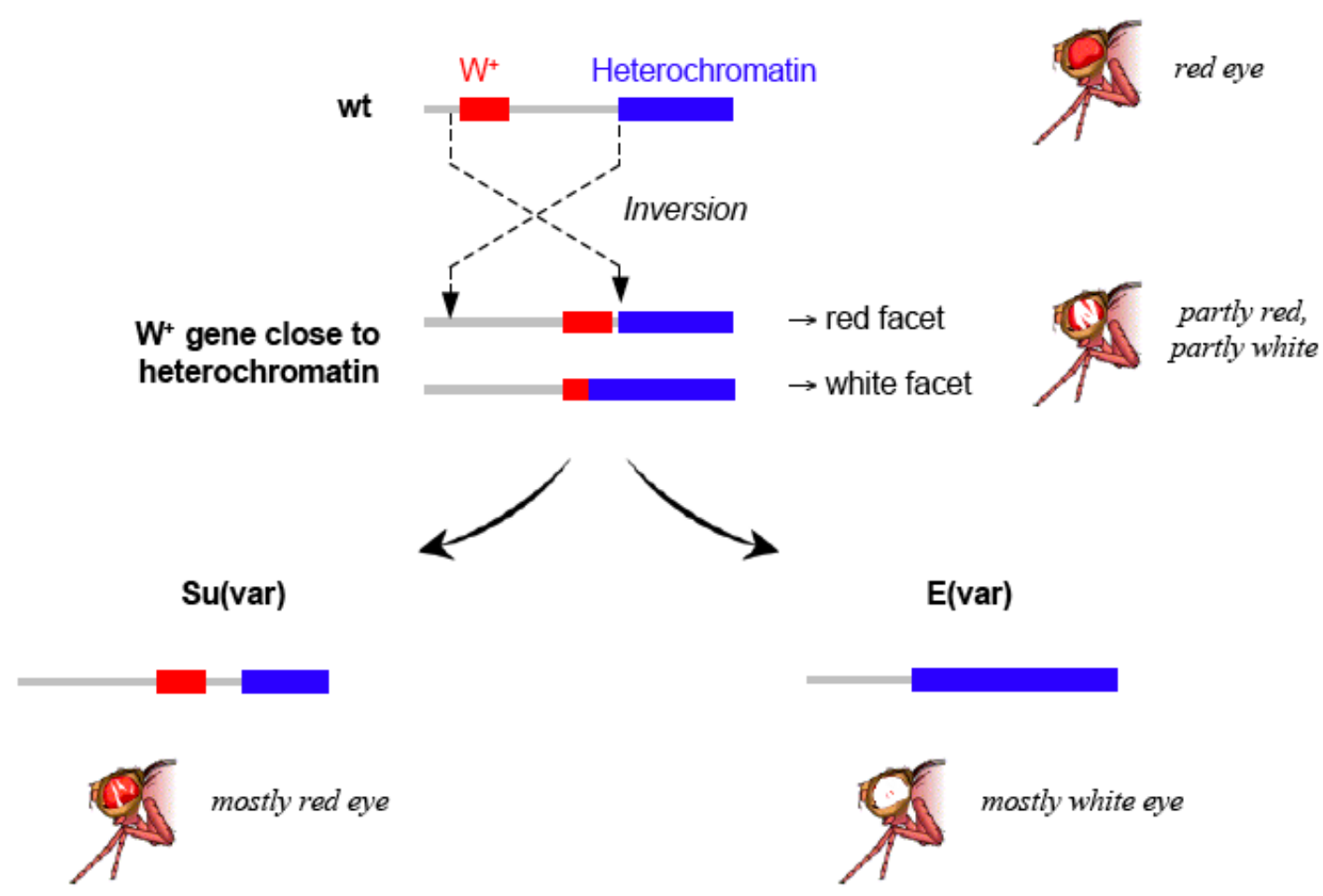

Figure 1-1: Position effect variegation in Drosophila melanogaster

In wild-type flies the gene responsible for the red eye pigmentation (white, $\mathrm{W}+$, depicted in red) is located within distal euchromatin. A gene inversion relocated the gene in close proximity to pericentromeric heterochromatin, resulting in a variegating phenotype: In some cells, the white gene is still regularly expressed, normally leading to red eye facets whereas in others it is silenced by heterochromatin spreading, causing white eye facets (Dormann, 2009).

Because the variegating phenotype is caused by a change in the position of the gene within the chromosome, this phenomenon is referred to as position-effect variegation (PEV). PEV has been used as a model system to systematically screen for factors that positively or negatively regulate heterochromatin formation.

Approximately 120 loci with roles as dominant Suppressors (Su(var)s) or Enhancers (E(var)s) of PEV have been identified so far in genetic screens (Reuter et al., 1987; Wustmann et al., 1989), but the expected number is likely to be higher since the genome 
has not yet been saturated for modifiers of PEV. Molecular analyses of several dozen modifier genes suggest diverse functions and confirm the expectation that only a subset is likely to be directly involved in heterochromatin formation.

\subsection{Chromatin}

The genetic information of eukaryotic cells is stored in the form of DNA, which reaches the length of 2 meters in human cells (Avery et al., 1944). To fit into the cell nucleus organization and compaction of DNA 'fibers' is required. Association of DNA with a set of nuclear proteins mediates this task. The resulting structures are called chromatin (Figure 1-2), the physiological form of all genetic and inheritable information in eukaryotic systems (Felsenfeld and Groudine, 2003).

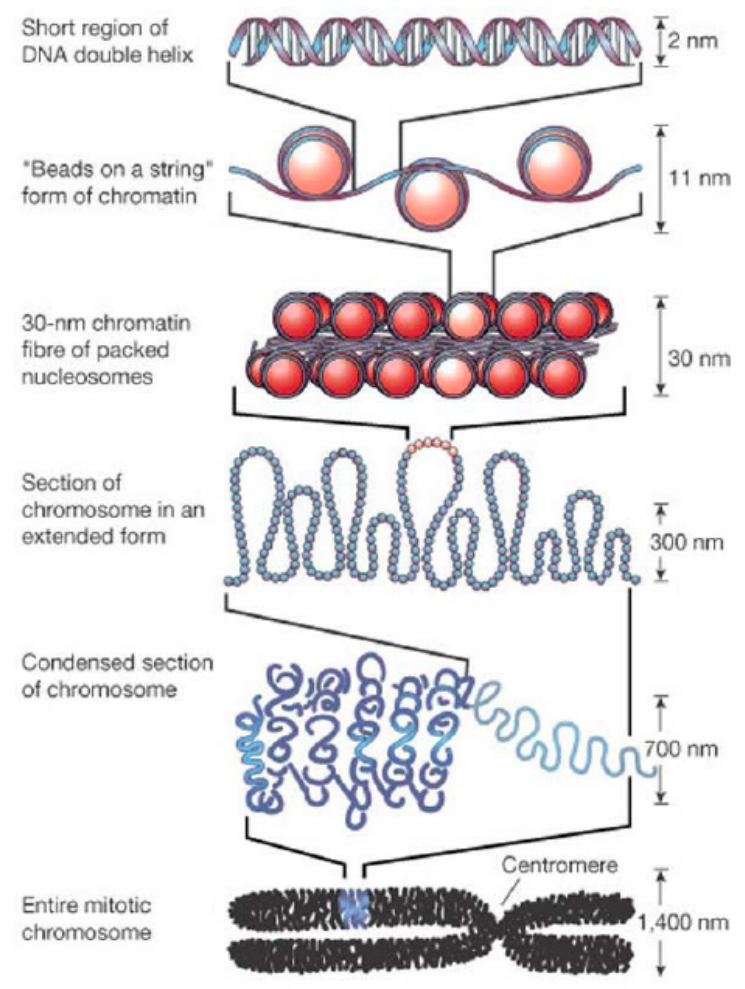

Figure 1-2: The organization of DNA within the cell 
The double helix of DNA is wrapped around octamers of histone molecules, forming nucleosomes in a 'beads on a string' formation. Further folding into a $30 \mathrm{~nm}$ fiber and higherorder structures compacts DNA more than 10,000-fold (adapted from (Felsenfeld and Groudine, 2003).

Chromatin consists of different functional areas and domains first defined in cytological studies by Heitz et al. as euchromatin and heterochromatin (Heitz, 1928). Heterochromatin retains its deep staining, condensed appearance throughout the cell cycle, including interphase, whereas euchromatin appears diffuse during interphase. Euchromatin has an open, accessible conformation, replicates early in S-phase, and contains the majority of active genes. Heterochromatin, in contrast, is tightly compacted, replicates late in the cell cycle, and contains very few active genes. It can be divided into constitutive heterochromatin which includes regions of the genome that are heterochromatic in all types of cells and at all times (e.g. centromeres, pericentric regions and telomeres) and facultative heterochromatin, which can change its status during development or differentiation.

\subsubsection{Nucleosomes and histones}

The basic repeating element of chromatin is the nucleosome (Kornberg, 1974; Olins and Olins, 1974), which consists of 147 bp of DNA wrapped around an octamer of histone proteins - two copies each of the histones H2A, H2B, H3 and H4 (Figure 1-3) (Luger et al., 1997). In addition, a linker histone H1 (and, in some organisms, H5), interacts with the nucleosomal core as well as with the interconnecting linker DNA to form higher levels of chromatin organization and architecture (Thomas, 1999). 

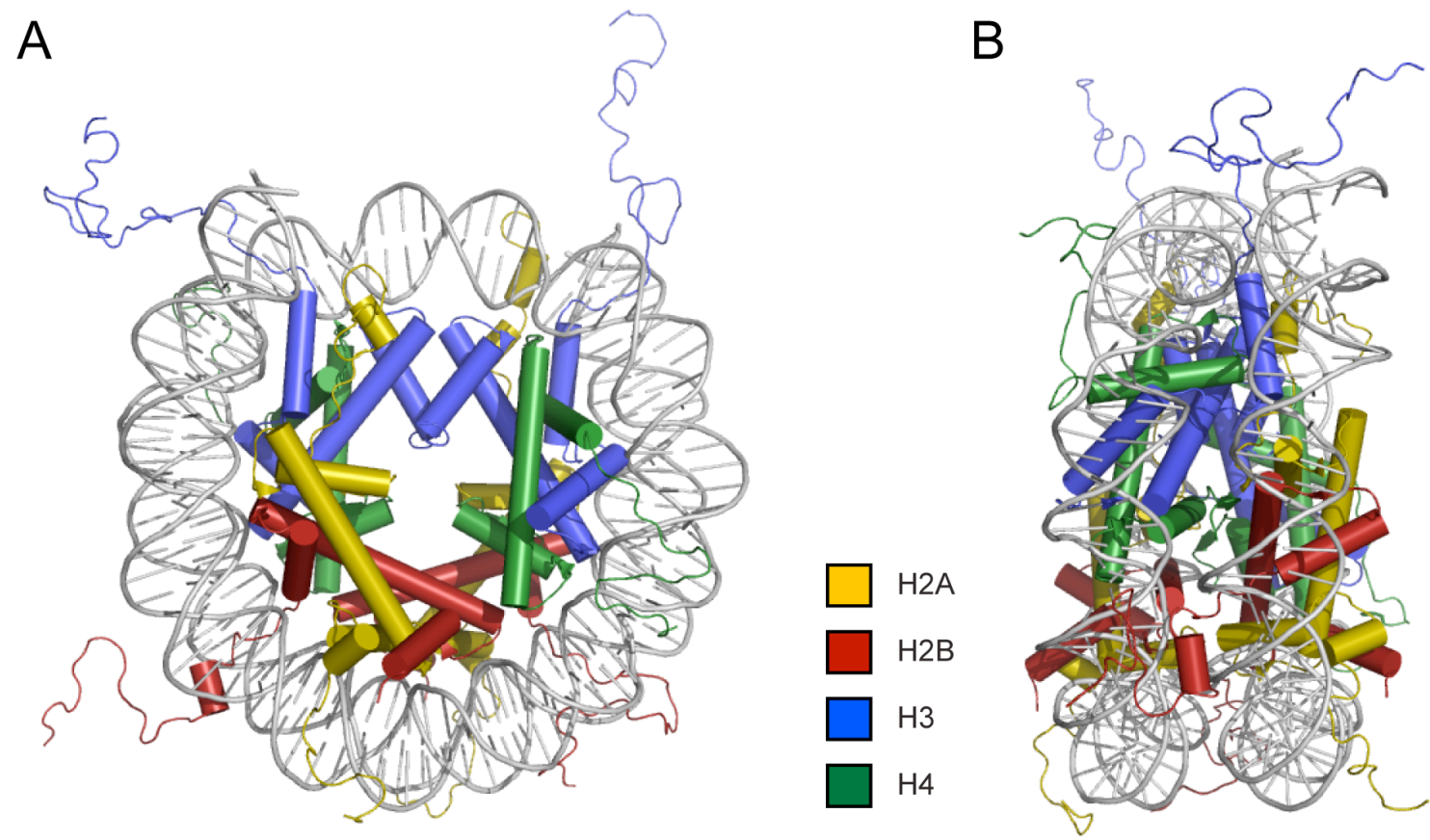

Figure 1-3: Structure of the nucleosome core particle

A, Crystal structure of the nucleosomal core particle, front view. DNA is shown in grey, core histones are depicted in the indicated colors. $\alpha$-helical structures are shown as cylinders,. B, Same structure in side view (Davey et al., 2002).

Histones represent a highly conserved family of small basic proteins containing many lysine and arginine residues in their N-terminal tails. Structurally, they consist of a 'helix turn helix turn helix' motif called 'histone fold' and relatively unstructured 'histonetails'. In solution histone $\mathrm{H} 2 \mathrm{~A}-\mathrm{H} 2 \mathrm{~B}$ and $\mathrm{H} 3-\mathrm{H} 4$ heterodimers are formed by head-to-tail association of the histone-fold-helices (Arents et al., 1991). The octamer is held together by three four-helix bundles between the above mentioned histone heterodimers: one H3$\mathrm{H} 3$ association forms the $(\mathrm{H} 3-\mathrm{H} 4)_{2}$ tetramer and two H2B-H4 interactions connect the (H3-H4) $)_{2}$ tetramer with two H2A-H2B dimers. The DNA is wrapped around the resulting globular structure mainly by interaction between the basic histones and the negatively charged DNA-backbone (Luger et al., 1997). The flexible N-terminal histone tails protrude outwards from the nucleosome core (Davey et al., 2002; Luger et al., 1997) making them available for interactions with non-histone chromatin factors or with adjacent nucleosomes (Angelov et al., 2001; Bertin et al., 2007; Mangenot et al., 2002). 


\subsubsection{Regulatory role of chromatin}

Chromatin is not only a protective and static scaffold for storage of genetic information. Dynamic changes in chromatin are involved in regulation of all genomic processes such as transcription, replication, mitotic chromosome condensation, and recombination as well as apoptosis and DNA repair (Felsenfeld and Groudine, 2003; Prigent and Dimitrov, 2003; van Attikum and Gasser, 2009).

Chromatin function is regulated by changes in chromatin structure and through the recruitment of specific effector proteins. Structural changes such as chromatin compaction or decondensation significantly affect the accessibility of DNA and thus can control various processes that require access to the DNA template (Hansen, 2002). Changes in chromatin structure can be achieved through chromatin remodeling complexes, the incorporation of histone variants and the addition or removal of posttranslational modifications of histone proteins (Allis, 2007). Several distinct posttranslational modifications on chromatin including covalent modifications of DNA and histones serve as epigenetic marks to recruit specific nuclear factors that in turn mediate downstream functions (Seet et al., 2006; Strahl and Allis, 2000; Taverna et al., 2007).

DNA methylation provides the most direct epigenetic mechanism for the maintenance of the repressed state. Methylation of DNA within the mammalian genome is catalyzed by a family of DNA (cytosine-5)-methyltransferases and occurs primarily at $\mathrm{CpG}$ dinucleotides. CpG methylation, especially within the promoter region of genes, is associated with stable transcriptional repression and plays important roles for Xchromosome inactivation and genomic imprinting (Bestor, 2000; Reik et al., 2001).

The N-terminal tails of histones are targets for a number of post-translational modifications (Fischle et al., 2003b). Covalent histone modifications are proposed to 
provide a storage mechanism for mitotically and meiotically inheritable information that can be 'read' by various effector proteins. By regulating access to underlying DNA, histone modifications and effector proteins dictate correct cell type specific gene expression patterns (Jenuwein and Allis, 2001).

\subsubsection{Histone modifications}

Histones can be extensively post-translationally modified (Allfrey, 1966). Over the last decades, an enormous number of distinct posttranslational modification types and sites have been identified on histones ("marks"), especially since the introduction of mass spectrometric approaches. In particular, the histone tails have been found to be subject to a great variety and high density of posttranslational modifications. Lysine residues in histones can be modified by acetylation (Kac), (mono-, di-, or tri-) methylation (Kme1/2/3), ubiquitylation and sumoylation (Kub and Ksu); arginine residues can be (mono-, and symmetrically/asymmetrically di-) methylated (Rme1/2), serine and threonine residues can be phosphorylated ( $\mathrm{Sph}$ and $\mathrm{Tph}$ ), and glutamate residues can be ADP-ribosylated (Kouzarides, 2007; Peterson, 2004). Close to a hundred individual histone marks have been identified to date, about half of which have been confirmed by independent experimental methods.

The complexity of histone modifications gives an enormous potential for functional responses. Histone acetylation, for example loosens chromatin packing and correlates with transcriptional activation, whereas histone deacetylation is generally associated with transcriptional repression (Struhl, 1998). Notably, distinct histone modifications may act sequentially or in combination to form a 'histone code' that is read by other proteins to bring about distinct downstream events. Strictly speaking the histone code hypothesis predicts that a pre-existing modification affects subsequent modifications on histone tails and that these modifications serve as marks for the recruitment of different proteins or protein complexes to regulate diverse chromatin functions, such as gene expression, DNA replication and chromosome segregation (Jenuwein and Allis, 2001). Furthermore 
epigenetic marks are important for mammalian telomere formation (Grunstein, 1997) and position effect variegation (Weiler and Wakimoto, 1995).

\subsubsection{Histone modifying enzymes}

Several distinct classes of enzymes can modify histones at several sites. Among the enzymes that establish histone marks ("writers") are histone acetyltransferases (HATs) (Sterner and Berger, 2000), histone kinases (Nowak and Corces, 2004), histone methyltransferases (HMTases) (Qian and Zhou, 2006; Zhang and Reinberg, 2001), and enzymes that mediate histone ubiquitylation (Weake and Workman, 2008), sumoylation (Nathan et al., 2006), and ADP-ribosylation (Hassa et al., 2006) (Table 1-1).

\begin{tabular}{l|l|l|l}
\hline Histone & Site & Enzyme & function \\
\hline \multirow{2}{*}{ H2A } & K5ac & Tip60, p300 & Transcriptional activation \\
\cline { 2 - 4 } & K119ub1 & RING1B & UV damage response \\
\hline \multirow{4}{*}{ H2B } & S14ph & Mst1 & Apoptosis \\
\hline \multirow{4}{*}{ H3 } & K4me1 & SET7/Set9 & Transcriptional activation \\
\cline { 2 - 4 } & K4me3 & SMYD3 & Transcriptional activation \\
\cline { 2 - 4 } & K9ac & SRC1 & Nuclear receptor coactivation \\
\cline { 2 - 4 } & S10ph & AuroraB & Mitotic chromosome condensation \\
\cline { 2 - 4 } & K27me3 & EZH2 & X-chromosome inactivation \\
\hline \multirow{3}{*}{ H4 } & R3me & PRMT1 & Transcriptional activation \\
\cline { 2 - 4 } & K5ac & P300 & Transcriptional activation \\
\cline { 2 - 4 } & K20me3 & Suv4-20h1/h2 & Transcriptional repression \\
\hline
\end{tabular}

Table 1-1: Examples of histone marks, the responsible enzymes and their functional context in mammals

Adapted from Allis et al., 2007. For references and a more complete list, see there. 
Most histone marks have been shown to be reversible. Enzymes that can remove specific histone modifications ("erasers") include histone deacetylases (HDACs) (Holbert and Marmorstein, 2005), phosphatases (Nowak and Corces, 2004) and lysine demethylases (Agger et al., 2008). Arginine methylation can be reversed by demethylation or deimination (Chang et al., 2007). In addition proteolytic clipping of histone tails (Allis et al., 1980; Duncan et al., 2008) and histone replacement (Ahmad and Henikoff, 2002) result in the removal of histone marks.

\subsubsection{Distribution of histone modifications along chromosomes}

Large scale genome-wide approaches such as amplification of immunoprecipitated DNA and hybridization to tiling oligonucleotide microarrays (ChIP-chip) (Bernstein et al., 2006; Heintzman et al., 2009) or direct massive parallel sequencing of short fragments of ChIP DNA (ChIP-seq) (Barski et al., 2007; Mikkelsen et al., 2007) have been used to map histone modifications. In these studies, distinctive sets of modifications have been associated with different functional regions along the mammalian chromosomes (Figure 1-4). The spatial distribution can be divided in two general classes: (i) large arrays of modified nucleosomes spanning several kb (Figure 1-4); and (ii) a few highly localised modified nucleosomes occurring in punctuate peaks within $1 \mathrm{~kb}$ (Figure 1-4).

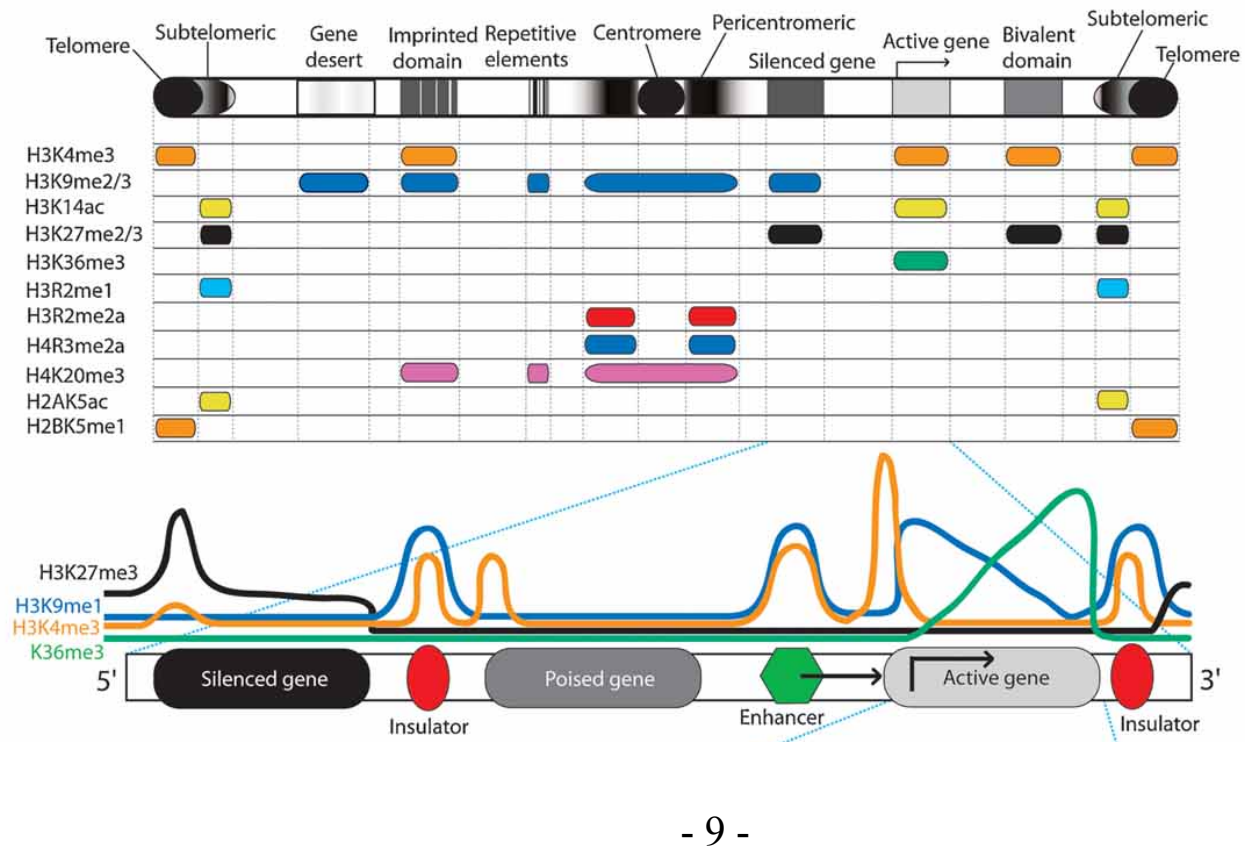


Figure 1-4: Histone modifications across a typical mammalian chromosome

Schematic representation of histone modifications across a typical mammalian chromosome and composite profile of typical histone modifications associated with silenced, poised, active genes and associated regulatory elements. Ac: acetylation, me1/me2/me3: mono/di/trimethylation, me2a: asymmetrical dimethylation (Wang et al., 2009).

A large portion of mammalian genomes is composed of non-genic chromosomal features that are derived from DNA transposons, retrotransposons, satellite repeats and structures that form telomeres and centromeres (Lander et al., 2001). These are largely constitutive heterochromatin features, associated with repressive modifications (Martens et al., 2005) (Barski et al., 2007; Mikkelsen et al., 2007).

Pericentromeric heterochromatin for example, is gene-poor, rich in repetitive satellite elements and interspersed with long and short interspersed nuclear elements (LINEs and SINEs, respectively). It is enriched in H3K9me3, H4K20me3 and H3K27me1 but is also associated with H3R2me2a and H4R3me2a (Figure 1-4 a) (Peters et al., 2003; Rosenfeld et al., 2009). Consistent with the presence of H3R $2 \mathrm{me} 2 \mathrm{a}$, which has been reported to inhibit H3K4 methylation and binding of H3K4me3-effectors, H3K4me3 is excluded from pericentromeric regions (Guccione et al., 2007; Iberg et al., 2008).

Genic regions including genes, promoters, enhancers and insulators are characterized by complex short-range histone modification patterns. Silent genes can be distinguished from non-genic regions by a greater enrichment of H3K27me3 (Rosenfeld et al., 2009). These regions are also associated with $\mathrm{H} 3 \mathrm{~K} 9 \mathrm{me} 2 / 3$ but lack H4K20me3 compared to centromeric regions (Rosenfeld et al., 2009). Promoters of silent CpG-rich genes are often associated with $\mathrm{H} 3 \mathrm{~K} 27 \mathrm{me} 3$ in conjunction with $\mathrm{H} 3 \mathrm{~K} 4 \mathrm{me}$, while those of inactive CpG-poor genes are devoid of H3K4me3 (Mikkelsen et al., 2007).

Transcriptionally active areas of the genome are largely associated with acetylation of lysines or methylation of H3K4 and H3K36 (Lee and Mahadevan, 2009; Wang et al., 2009). While H4K12ac and H3K36ac cover the whole gene region, other modifications are distributed only around the transcriptional start site (H3K4me, H3K9ac and H3K18ac) or the body of genes (H3K36me3) (Lee and Mahadevan, 2009; Wang et al., 2009). 


\subsubsection{Reading the histone marks}

Histone modifications either modulate chromatin structure directly ('cis') or they act in 'trans' by recruiting binding partners that induce and direct downstream functions (Figure 1-5, (Allis, 2007).

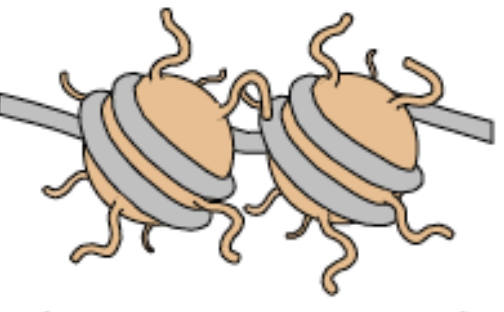

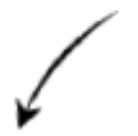

'cis'

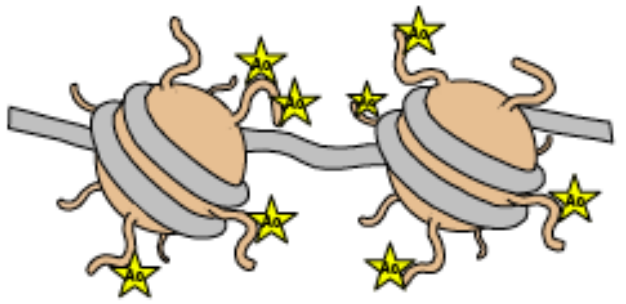

'trans'

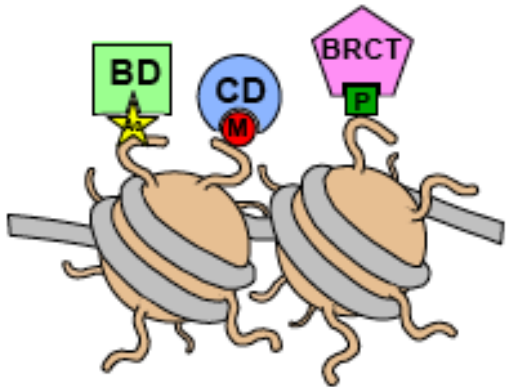

Figure 1-5: Molecular mechanisms of histone modifications

Histone modifications fulfill their function by two general mechanisms. They either change the net charge of the histone tail, which affects inter/intranucleosomal contacts and results in structural changes of the chromatin fiber ("cis" mechanisms, left). Or they recruit proteins with specific binding modules, which mediate downstream functions ("trans" mechanisms, right). Ac: acetylation. M: methylation. P: phosphorylation. BD: bromodomain. CD: chromodomain. BRCT: Breast-cancer-susceptibility protein-1 Cterminal domain (Dormann, 2009) 
Modifications may affect higher-order chromatin structure by affecting the interaction of histones with DNA or the contact between different histones in adjacent nucleosomes. Acetylation has the greatest potential to unfold chromatin since it neutralizes the positive charge of the lysine residue. Chromatin condensation can be blocked by a certain amount of acetylation in general (Hansen, 2006; Hansen et al., 1998). In vitro studies have shown that acetylation of H4K16 has a negative effect on the formation of a 30-nanometer fiber and the generation of higher-order structures (Shogren-Knaak et al., 2006) whereas deacetylation of H4K16 facilitates compaction of chromatin (Johnson et al., 2009). Phosphorylation might also have consequences on chromatin compaction via charge changes (Johansen and Johansen, 2006).

In the trans mechanism, effector mediated changes are elicited in the chromatin fiber due to specific binding events that couple a particular histone modification with a cognate non-histone binding partner. Effector proteins may alter the properties of chromatin by crosslinking two or more nucleosomes (Francis et al., 2004; Nielsen et al., 2001a; Zhao et al., 2000), by affecting the RNA polymerase complex and related factors (Vermeulen et al., 2007) or by recruiting chromatin remodeling or further chemical modification activities (Jenuwein and Allis, 2001).

A number of specific binding proteins ("readers") have been identified over the last years. Bromodomains may specifically engage acetylated lysines, particularly in histone sequence contexts (Dhalluin et al., 1999). These are found in transcription factors and chromatin remodeling complexes that are associated with active transcription (Zeng and Zhou, 2002). Recruitment of bromodomain containing factors to promoter regions has been demonstrated to activate transcription in several model systems (Agalioti et al., 2002; Agalioti et al., 2000; Cosma et al., 1999; Mujtaba et al., 2007). Chromo domains act as binding modules for methylated lysines. Proteins containing a chromo domain include Heterochromatin Protein 1 (HP1), which recognizes H3K9me2/3 and Polycomb, a H3K27me3 binding factor. HP1 is involved in gene silencing and heterochromatinization (Jacobs and Khorasanizadeh, 2002; Jacobs et al., 2001; Lachner et al., 2001). Polycomb mediates repression and has important functions in development 
(Fischle et al., 2003c).

Plant homeo domains (PHD), Tudor domains (TD) and malignant brain tumor(MBT) repeat domains, which belong, along with the Chromo domains, to the 'royal family' represent additional methyl-lysines binding modules (Adams-Cioaba and Min, 2009). Furthermore, proteins/domains that recognize phosphorylation such as the 14-3-3 protein family and WD40 domains (Seet et al., 2006), as well as ubiquitin binding domains (Hofmann, 2009) have been characterized as histone modification binding proteins.

The abundance of post-translational modifications on the histone tail makes 'crosstalk' between modifications very likely. First, the binding of a protein can be affected by an adjacent modification as has been shown for the H3K9me3 binding of HP1, which is disrupted by H3S10 phosphorylation (Fischle et al., 2005). Second, the modification of the recognition site may influence substrate recognition or the catalytic activity of an enzyme. For example the acetyltransferase GCN5 recognizes H3 more effectively when it is phosphorylated at H3S10 (Clements et al., 2003). Also cis/trans isomerization of H3P38 affects methylation of H3K36 by Set2 (Nelson et al., 2006). Communication between modifications can also occur when modifications are on different histone tails as in the case of $\mathrm{H} 2 \mathrm{~B}$ ubiquitination being required for $\mathrm{H} 3 \mathrm{~K} 4$ and $\mathrm{H} 3 \mathrm{~K} 79$ methylation.

\subsubsection{Methylation of histone $\mathrm{H3}$ lysine 9}

\section{Establishment of H3K9 methylation}

H3K9 is methylated by diverse HMTases which can interact with a variety of factors. In mammals, the histone methyltransferases G9a and G9a-like protein GLP monomethylate lysine 9 of histone H3 (Collins et al., 2008). Di- or trimethylation of histone H3 lysine 9 is mediated by Suv39h1/h2 as well as ESET/SETDB1 histone methyltransferase (Kouzarides, 2007; Wang et al., 2003). Interaction of the histone methyltransferases with DNA-binding proteins as well as with small RNAs is also involved in the methylation process (Grewal and Elgin, 2007). SETDB1 is mainly found in euchromatic regions, 
where it participates in gene silencing (Schultz et al., 2002). In contrast, Suv39-like enzymes mainly localize to pericentromeric heterochromatin. Suv39h1/h2 double knockout mice fail to show H3K9 trimethylation at these loci (Aagaard et al., 1999; Peters et al., 2003). Therefore, it is likely that Suv39h1/h2 is the main HMTase establishing $\mathrm{H} 3 \mathrm{~K} 9$ trimethylation at heterochromatic regions.

The first demethylating enzyme of H3K9 methylation that has been described is LSD1, which acts in a complex with the androgen receptor and activates transcription (Metzger et al., 2005). Other H3K9 demethylases include the jumonji proteins JHDM2A, JMJD2A/JHDM3A, JMJD2B, JMJD2C/GASC1 and JMJD2D (Kouzarides, 2007). The exact mechanism and occurrences of these demethylation events are not well understood so far.

\section{Biological functions of trimethylated H3K9}

H3K9 methylation has mainly been linked to heterochromatic domains and gene silencing. For example, Suv39h1 mediated methylation is associated with transcriptionally inert regions at pericentric heterochromatin in mammals (Lachner et al., 2001; Peters et al., 2001). Loss of the Suv39h HMTase activities in mutant mice impairs pericentric H3K9 methylation but does not affect the broad methylation at the chromosome arms (Peters et al., 2001). Mice lacking Suv39h1/h2 enzymes display impaired viability, overall genomic instability in both mitotic and meiotic cells, an increased risk for B-cell lymphomas and complete spermatogenic failure, suggesting a potential role in tumor suppression, mitosis and meiosis (Peters et al., 2001).

H3K9 methylation is not restricted to constitutive heterochromatin. The Suv39h1 HMTase is also recruited to euchromatic targets, through interaction with HP1 and by the tumor suppressor retinoblastoma protein $(\mathrm{pRb})$, where it is also involved in gene repression (L. Vandel, 2001; Nielsen et al., 2001b).

Notably, H3K9 methylation has been shown to have a direct impact on DNA 
methylation. One of the three mammalian isoforms of HP1, HP1 $\alpha$ interacts with the DNA methyltransferase DNMT3b and targets it to heterochromatic foci. However, in Suv39h1/h2 double-null cells it fails to localize to these regions. Consistently, Suv39h1/h2 knockout cells display an altered DNA methylation profile at major satellite repeats but not at other repeat sequences (Lehnertz et al., 2003).

How one modification can be involved in regulating so many functions at such distinct chromatin environments is not well understood. The induction of an H3K9 HMTasedependent chromatin configuration could require the combination of two or more signals that involve a defined pre-existing modification pattern of the associated nucleosomes and/or the presence of a 'specific' DNA or RNA sequence. Subsequently, the overall density of methylated nucleosomes might contribute to the distinct qualities of H3K9 methylation in constitutive versus facultative heterochromatin and in euchromatin (Maison et al., 2002; Peters et al., 2001).

\subsubsection{Heterochromatin Protein 1 (HP1)}

HP1 proteins consist of an N-terminal chromo domain (CD), a flexible hinge region and a C-terminal chromoshadow domain (CSD).

\section{HP1 chromo domain interactions}

The chromo domain was originally described as a domain of about 40 amino acids that was present in various proteins involved in chromatin organization and gene regulation (Koonin et al., 1995). The CD of HP1 is a binding module for H3K9me3 (Bannister et al., 2001; Jacobs et al., 2001; Lachner et al., 2001). Interestingly, the amino acid context of H3K9 ("ARKS") is found in identical or similar form at multiple other sites in histones and other proteins. The corresponding lysines have been found to be methylated in several of these instances: H3K27: ARKmeS binds Polycomb (Fischle et al., 2003c), H1K26: ARKmeS binds HP1 (Daujat, 2005), G9aK165: ARKmeT binds HP1 (Sampath 
et al., 2007). Additional modifications such as lysine acetylation and serine phosphorylation occurring in this sequence stretch suggest that ARKS and related sequences might act as "modification cassettes" which confer the ability for the recruitment of specific binding partners (Fischle et al., 2003a).

Disruption of the H3K9me2/3 - HP1 interaction by mutations in the CD or removal of the H3K9me mark results in mislocalization of HP1 in flies, S. pombe and mammals (Fischle et al., 2003c; Platero J.S., 1995; Platero et al., 1995; Stewart et al., 2005; Thiru et al., 2004). Thus, binding of the chromo domain to the modified $\mathrm{H} 3$ tail is essential for recruitment of HP1 to chromatin.

\section{HP1 chromoshadow domain interactions}

The structure of the chromoshadow domain bears some resemblance to the chromo domain (a three-stranded $\beta$ sheet packed against two $\alpha$ helices; (Cowieson et al., 2000). However, it does not engage a histone modification, but rather mediates a wide array of protein interactions. The HP1 CSD mediates homodimerization with the same HP1 isoform as well as heterodimerization between different HP1 isoforms (Brasher et al., 2000; Cowieson et al., 2000; Gaudin et al., 2001; Thiru et al., 2004).

A phage display screen carried out with the HP1 CSD identified a pentapeptide motif PXVXL (X = any amino acid) that interacts specifically with the chromo shadow domain (Smothers and Henikoff, 2000). Binding of proteins with the PXVXL motif to HP1 requires dimerization of the $\mathrm{CSD}$, which generates a hydrophobic groove that the peptide motif associates with (Thiru et al., 2004). The peptide motif has been shown to be present in many reported interaction partners of the HP1 CSD, for example in KAP-1 (Brasher et al., 2000), Su(var)3-7 (Delattre et al., 2000), CAF-1 p150 (Brasher et al., 2000), the TAFII130 component of TFIID (Vassallo and Tanese, 2002), and AF10 (Linder et al., 2001). This HP1 binding to transcriptional repressors could provide the means for initial recruitment of HP1 to specific regions of chromatin. $\mathrm{Rb}$, for example, has been reported to recruit HP1 $\beta$ to the cyclin E promoter and induce tri-methylation of H3K9 by Suv39h1 
(Nielsen et al., 2001b). Suv39h1 itself also interacts physically with HP1 via interaction with the CSD (Aagaard et al., 1999; Melcher et al., 2000).

The binding of HP1 to a variety of interaction partners such as DNA methyltransferases, histone methyltransferases and transcriptional repressors (Brasher et al., 2000; Lehnertz et al., 2003; Schotta et al., 2004) suggests a role of HP1 is to serve as a platform for the recruitment of downstream factors to $\mathrm{H} 3 \mathrm{~K} 9 \mathrm{me} 3$. However, as for the modification itself it is unclear how HP1 distinguishes between different interaction partners to exert its function in the different contexts.

\section{Model for heterochromatin spreading}

As described above, Suv39h1 functionally interacts with the chromo domain of HP1. Suv39h1 methylates H3K9 and thus generates a binding site for the HP1 CD that is essential for HP1 recruitment to chromatin. The interaction between Suv39h1 and the HP1 CSD suggests a mechanism for the spreading and maintenance of heterochromatic structures and epigenetic gene silencing. According to this model, Suv39h1, the histone H3 tail and HP1 collaborate to form a self-sustaining loop. Methylated H3K9me recruits HP1, which in turn directs more Suv39h1 histone methyltransferase to chromatin, enabling further methylation (Figure 1-6).

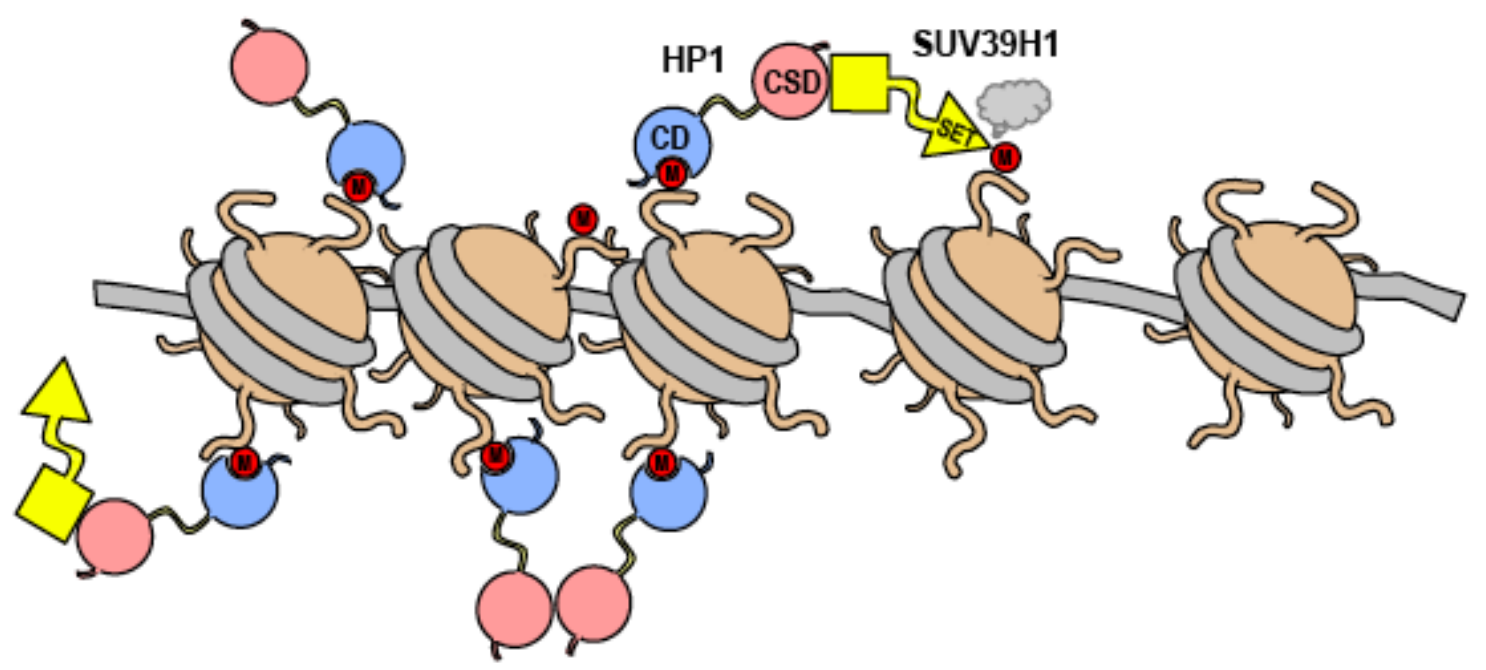




\section{Figure 1-6: Model for the spreading of heterochromatin}

The histone mark H3K9me (small red circle "M") recruits the effector protein HP1 via its chromo domain (CD) to chromatin. HP1 crosslinks nucleosomes and recruits effector proteins such as the HMTase Suv39h1 (in yellow). Upon recruitment to chromatin, Suv39h1 methylates through its SET domain adjacent unmethylated $\mathrm{H} 3$ tails at lysine 9, forming new H3K9me binding sites for HP1. Thus, this three-component system could explain spreading and maintenance of heterochromatic gene silencing (Dormann, 2009).

Subsequently, H3K9me3 bound HP-1 dimerizes and interacts with many nuclear proteins via its chromoshadow domain. That may lead to downstream functions either directly by interconnecting nucleosomes to compact chromatin (Soeroes, 2010) or indirectly by recruiting factors such as Suv4-20 (trimethylates H4K20), TRIM28 (involved in transcriptional repression) and DNMT3a/b (a DNA methyltransferase) (Lehnertz et al., 2003; Schotta et al., 2004). 


\subsection{Activity-dependent neuroprotective protein (ADNP)}

\subsubsection{ADNP discovery and structure}

ADNP was originally cloned from P19 mouse carcinoma cells, differentiated into neuroglial cells (Bassan et al., 1999). The hADNP gene spans $40 \mathrm{~kb}$ and includes 5 exons and 4 introns with alternative splicing of an untranslated second exon. Protein variants resulting from alternative splicing have not yet been reported. Sequence comparison with human ADNP from a fetal brain cDNA library (Zamostiano et al., 2001) indicated $90 \%$ identity at the mRNA level, suggesting a very high evolutionary conservation, which also extends to the clade of euteleostomi (http://www.ncbi.nlm.nih.gov/homologene, Figure $1-7)$.

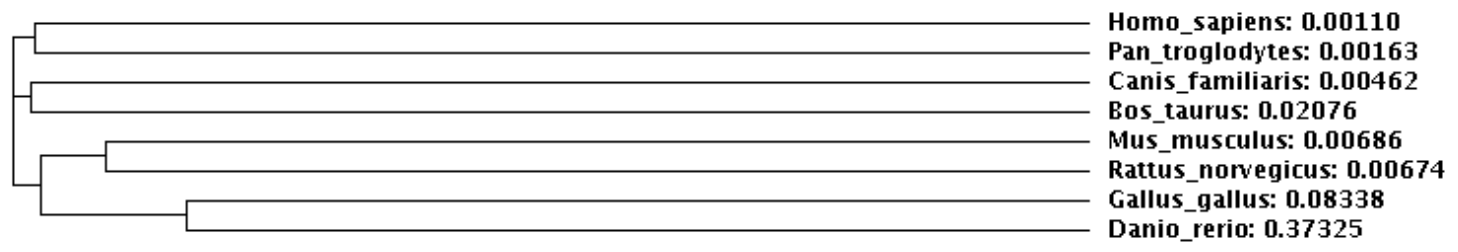

Figure 1-7: phylogenetic tree of ADNP homologs in different species.

(http://www.ncbi.nlm.nih.gov/homologene)

The predicted structure of the $124 \mathrm{kDa}$ protein contains 9 zinc fingers, a proline-rich region, a nuclear bipartite localization signal, cellular export and import signals, and a homeobox domain profile (Table 1-2). ADNP exhibits sequence homology to the engrailed homeoprotein, containing a leucine-rich sequence within the homeobox profile that has been shown to be necessary for nuclear export and secretion (Furman et al., 2004; Gozes and Brenneman, 2000; Gozes et al., 2000). Therefore, ADNP may play a role in transcription, cytoplasmic and extracellular pathways. Besides zinc fingers and the 
homeodomain, no other regions of similarity are predicted. Therefore it is impossible to derive assumptions about the protein function from domain structure.

\begin{tabular}{|l|r|r|l|}
\hline \multicolumn{3}{|l|}{ Zinc finger } \\
\hline Feature key & Position(s) & Length & Description \\
\hline Zinc finger & $74-97$ & 24 & C2H2-type 1 \\
\hline Zinc finger & $107-129$ & 23 & C2H2-type 2; atypical \\
\hline Zinc finger & $165-188$ & 24 & C2H2-type 3 \\
\hline Zinc finger & $221-244$ & 24 & C2H2-type 4 \\
\hline Zinc finger & $447-469$ & 23 & C2H2-type 5; atypical \\
\hline Zinc finger & $489-510$ & 22 & C2H2-type 6; atypical \\
\hline Zinc finger & $512-535$ & 24 & C2H2-type 7 \\
\hline Zinc finger & $622-647$ & 26 & C2H2-type 8; atypical \\
\hline Zinc finger & $662-686$ & 25 & C2H2-type 9; atypical \\
\hline DNA binding & $754-814$ & 61 & Homeobox \\
\hline Region & $354-361$ & 8 & Neuroprotective peptide (NAP) \\
\hline
\end{tabular}

Table 1-2: domains of human ADNP (Q9H2P0) as predicted by UniProtKB (http://www.uniprot.org/uniprot/Q9H2P0\#section_comments)

\subsubsection{ADNP expression and distribution}

\section{Tissue specific expression}

Human ADNP mRNA shows higher expression in the heart, skeletal muscle, kidney, placenta, microvascular endothelial cells and brain as compared to other tissues such as the colon or the small intestine (Zamostiano et al., 2001). Serial analysis of gene expression suggests increased expression in tumor tissues. 
In mice, Northern blot hybridizations have identified a unique 5.5-kb ADNP mRNA in the brain. Comparison of tissues reveals enrichment in brain-derived structures and low abundance in the lung, kidney, and intestine with slight increases in the testis (Bassan et al., 1999).

\section{Subcellular distribution}

Cell fractionation, gel electrophoresis, immunoblotting, and immunocytochemistry reveal ADNP-like immunoreactivity in both the cytoplasmic and in the nuclear cell fractions of astrocytes (Furman et al., 2004).

In the extracellular milieu of astrocytes, ADNP-like immunoreactivity is detected as well. Its content is increased with the vasoactive intestinal peptide (VIP), which is known to activate astrocytes to secrete neuroprotective/neurotrophic factors. This observation suggests that ADNP constitutes part of this VIP-stimulated protective pathway (Furman et al., 2004).

Homology between the engrailed cytoplasmic import sequence and ADNP indicates ADNP may be internalized by other cells, such as neurons after being secreted from glial cells (Furman et al., 2004).

ADNP-like immunoreactivity in the cytoplasm colocalizes with tubulin/microtubule-like immunoreactivity, which correlates with microtubules being key components of the neuron/brain (Furman et al., 2004). Neuroprotection by ADNP is conferred by NAP, an 8-amino acid fragment of ADNP (Bassan et al., 1999). Further studies identified tubulin as an NAP target for astrocyte (Divinski et al., 2004) and neuronal protection (Divinski et al., 2006). NAP appears to show some selectivity in binding to the neuron enriched beta III isoform (Burkhart et al., 2001; Divinski et al., 2006; Katsetos et al., 2003) and interacts with brain tubulin but not with tubulin from fibroblasts (Divinski et al., 2006). 
In the nucleus, ADNP has been identified as one of the proteins that interact with the interferon-alpha promoter ( $\mathrm{Qu}$ et al., 2005). Interferon-alpha has been associated with immunomodulation/autoimmunity and is used as a therapeutic agent against hepatitis $\mathrm{C}$ virus (Selmi et al., 2006). Association of ADNP with nuclear compartments such as nucleoli or pericentromeric heterochromatin has not yet been investigated.

\subsubsection{ADNP Function}

\section{Neuroprotective function of ADNP}

To test ADNP function in neuronal systems it has been fused to VP22, a viral protein that facilitates penetration through cellular membranes, potentially enhancing the intrinsic cellular penetration signal in ADNP (Steingart and Gozes, 2006). When incubated with pheochromocytoma cells (PC12), a neuronal model system, VP22-ADNP protects against beta amyloid peptide toxicity and oxidative stress $\left(\mathrm{H}_{2} \mathrm{O}_{2}\right)$. Furthermore, the p53 level decreases in the stressed cells, whereas in non-treated cells a p53 increase under oxidative stress has been observed. Interestingly, VIP analogues that provide protection to PC12 cells against $\mathrm{H}_{2} \mathrm{O}_{2}$ induce a parallel increase in ADNP expression (Sigalov et al., 2000). These results suggest that exposure of postmitotic cells (such as neurons) to environmental stress and injurious conditions results in p53 increases and enhances cellular demise which may be rescued by increased ADNP levels.

An increase in ADNP in injured brains is suggested to be a part of an endogenous compensatory mechanism and VIP treatment of a mouse macrophage cell line causes an increase of ADNP expression (Beni-Adani et al., 2001; Quintana et al., 2006). Thus, ADNP may be activated as a protective mechanism of neuroimmune interactions. 


\section{ADNP function in embryogenesis}

Mouse ADNP has been detected at the time of neural tube closure at E7.5 and increased on E9.5. Expression is augmented in the brain (E12.5), sustained throughout embryogenesis and regulated by VIP (Pinhasov et al., 2003). Analysis in ADNP knockout mice reveals failure of cranial neural tube closure and death on E8.5-9.5 (Pinhasov et al., 2003). In turn, the neuroprotective peptide motif of ADNP, NAP (Bassan et al., 1999; Gozes et al., 2005) has been shown to enhance neural tube closure, which is compromised in the case of alcohol exposure, as observed in conditions of fetal alcohol intoxication (Chen and Charness, 2008; Sari and Gozes, 2006).

The expression of Oct4, a gene associated with germ-line maintenance is down regulated and the expression of Pax6, a gene crucial for cerebral cortex formation, is abolished in the brain primordial tissue in ADNP knockout embryos. This observation indicates a mechanism of ADNP action on brain formation, inhibiting germ-line division while activating morphogenesis (Pinhasov et al., 2003).

\section{Nuclear function of ADNP}

Down-regulation of ADNP up-regulates the tumor suppressor p53 and causes reduced cell viability. Thus, ADNP is implicated in maintaining cell survival, perhaps through modulation of p53 (Zamostiano et al., 2001).

Co-immunoprecipitation experiments identify BRG1, BAF250a, and BAF170, all components of the SWI/SNF (mating type switching/sucrose nonfermenting) complex, as interaction partners of ADNP. Mammalian SWI/SNF is a multiprotein chromatinremodeling complex that is composed of at least 10 elements existing in two distinct forms that contain either BAF250 and BRG1 or BRM (termed BAF) or BAF180 and BRG1 (termed PBAF) (Simone, 2006). ADNP associates with the BAF complex containing the BRG1 core motor subunit. ADNP down-regulation in HEK293 cells 
results in reduced cell viability and microtubule reorganization and changes in cell morphology. These morphological changes are closely associated with the SWI/SNF complex multifunctionality. In addition, ADNP association with neuronal maturation in pluripotent p19 cells that are differentiated into neuronal/glial phenotype (Mandel et al., 2007) correlates well with BRG1 (and by inference the SWI/SNF complex) being an essential regulator of neuronal differentiation (Eroglu et al., 2006) with direct interaction with Neurogenin1 and Neurod (Seo et al., 2005).

In addition to its transcriptional activator functions, the SWI/SNF complex has been described to interact with the retinoblastoma tumor suppressor (RB) in transcriptional repression at euchromatic sites in concert with the Suv39h1/HP1 complex (Nielsen et al., 2001b). At heterochromatic sites, Brg1 knockout causes a disruption of pericentromeric heterochromatin domains as shown with DNA, H3K9me3 and H4K20me3 stainings (Bourgo et al., 2009). Whether ADNP is involved in these heterochromatin functions has not been reported so far.

Morphological changes after ADNP down-regulation are also in line with a SWI/SNF complex function that is associated with cellular differentiation. BRG1 loss induces an altered cellular morphology and disruption in the organization of the actin cytoskeleton (Rosson et al., 2005). Mutations in the SWI/SNF complex result in changes in cell size and attachment area (Hill et al., 2004). 


\subsection{Objective of the thesis}

A main focus in chromatin research is trying to understand how enzyme systems that mediate histone modifications, histone modification marks, specific binding proteins of such marks, and other factors/components interplay to establish and maintain distinct functional domains of chromatin such as euchromatin and heterochromatin. Parts of this process have been described. For example, Suv39h1 (the H3K9me3 methyltransferase), the H3K9me3 mark, and HP1 (a H3K9me3 binding protein) seem to be involved in the formation of heterochromatin. However, our insights into the biochemistry of the different states of chromatin and the complement of factors that mediate these states are still very limited.

To get a more detailed picture of the Suv39h/H3K9me3/HP1 pathway I used modern mass spectrometric methods to identify effector proteins and accessory factors that are associated with H3K9me3 modified chromatin. Factors that had not been described in a heterochromatin context so far were chosen for further characterization.

First, the mechanism by which such a protein is recruited to heterochromatin was investigated. I tested whether binding to $\mathrm{H} 3 \mathrm{~K} 9 \mathrm{me} 3$ occurs either by direct binding to that modification or indirectly by interaction with another H3K9me3 recognizing protein. Domains and interaction interfaces were then mapped to deepen the understanding of how specific factors are targeted to heterochromatin to mediate distinct functions.

In the second part, I addressed the function of the selected protein at pericentromeric heterochromatin. Therefore, the knockdown phenotype was analyzed for defects in heterochromatin features such as chromatin density, the level and distribution of histone modifications, DNA methylation and transcriptional inactivity.

These studies aimed to broaden our knowledge about how histone modifying enzymes, histone modifications and their binding partners interact to finally mediate distinct biological functions. 


\section{Material and Methods}

\subsection{Material}

\subsubsection{Chemicals}

\begin{tabular}{|c|c|c|c|}
\hline Chemical & Manufacturer & Chemical & Manufacturer \\
\hline 2-Mercaptoethanol & Sigma, Steinheim & Luciferin & PJK, Rehovot (Israel) \\
\hline $\begin{array}{l}\text { 4-(2-Hydroxyethyl)-1- } \\
\text { piperazineethanesulfonic } \\
\text { acid (HEPES) }\end{array}$ & & Magnesium chloride & \\
\hline $\begin{array}{l}\text { 4,6-Diamidino-2- } \\
\text { phenylindole (DAPI) }\end{array}$ & Sigma, Steinheim & $\begin{array}{l}\left(\mathrm{MgCl}_{2}\right) \\
\text { Magnesium } \quad \text { sulfate } \\
\left(\mathrm{MgSO}_{4}\right)\end{array}$ & Roth, Karlsruhe \\
\hline Acetic acid & Merck, Mannheim & Maleic acid & Sigma, Steinheim \\
\hline $\begin{array}{l}\text { Acrylamide / Bisacrylamide } \\
(37.5: 1)\end{array}$ & Merck, Mannheim & Methanol & Sigma, Steinheim \\
\hline $\begin{array}{l}\text { Adenosine 5'-triphosphate } \\
\text { (ATP) }\end{array}$ & Sigma, Steinheim & Milk powder & $\begin{array}{l}\text { Regilait, Saint-Martin- } \\
\text { Belle-Roche (France) }\end{array}$ \\
\hline Agar & Roth, Karlsruhe & Mowiol & Calbiocem, Darmstadt \\
\hline Agarose & Serva, Heidelberg & Non essential amino acids & Gibco, Muenchen \\
\hline Albumin, bovine (BSA) & $\begin{array}{l}\text { New England Biolabs, } \\
\text { Ibswich }\end{array}$ & Nonidet P-40 (NP-40) & Roche, Penzberg \\
\hline $\begin{array}{l}\text { Ammonium persulfate } \\
\text { (APS) }\end{array}$ & $\begin{array}{l}\text { AppliChem } \quad \mathrm{GmbH}, \\
\text { Darmstadt }\end{array}$ & Normal goat serum & $\begin{array}{l}\text { Bioscience International, } \\
\text { Maine (USA) }\end{array}$ \\
\hline Ampicillin & $\begin{array}{l}\text { AppliChem } \quad \mathrm{GmbH}, \\
\text { Darmstadt }\end{array}$ & Paraformaldehyde & Sigma, Steinheim \\
\hline Benzyl mercaptan & Sigma, Steinheim & $\begin{array}{l}\text { Penicillin Streptomycin } \\
100 \mathrm{x}\end{array}$ & Gibco, Muenchen \\
\hline Boric acid & Merck, Mannheim & Peptone & Roth, Karlsruhe \\
\hline Bromophenol blue & Serva, Heidelberg & Phenol & Sigma, Steinheim \\
\hline Calcium chloride $\left(\mathrm{CaCl}_{2}\right)$ & Roth, Karlsruhe & $\begin{array}{l}\text { Phenol/Chloroform/Isoamyl } \\
\text { alcohol }\end{array}$ & Roth, Karlsruhe \\
\hline Chloramphenicol & Amresco, Solon (USA) & PLB buffer & Promega, Madison \\
\hline Chloroform & Merck, Mannheim & Polyethylene glycole 8000 & Merck, Mannheim \\
\hline Coelenterazine & Invitrogen, Karlsruhe & Ponceau S & Sigma, Steinheim \\
\hline Coenzyme A & Invitrogen, Karlsruhe & Potassium chloride (KCl) & Roth, Karlsruhe \\
\hline Coomassie brilliant blue & BIO-RAD, Muenchen & $\begin{array}{l}\text { Potassium dihydrogen } \\
\text { phosphate }\left(\mathrm{KH}_{2} \mathrm{PO}_{4}\right) \\
\text { Potassium monohydrogen }\end{array}$ & Roth, Karlsruhe \\
\hline Dimethylsulfoxid (DMSO) & Sigma, Steinheim & phosphate $\left(\mathrm{K}_{2} \mathrm{HPO}_{4}\right)$ & Merck, Mannheim \\
\hline Dithiothreitol (DTT) & Alexis Biochemicals & Protease Inhibitor & Roche, Penzberg \\
\hline $\begin{array}{l}\text { DMEM GlutaMAXII } \\
\text { Pyruvate }]\end{array}$ & Gibco, Muenchen & Puromycin & $\begin{array}{l}\text { InvivoGen, San Diego } \\
\text { (USA) }\end{array}$ \\
\hline
\end{tabular}




\begin{tabular}{|c|c|c|c|}
\hline Chemical & Manufacturer & Chemical & Manufacturer \\
\hline & & & GE Healthcare, \\
\hline dNTPs & Invitrogen, Karlsruhe & $\mathrm{S}-[3 \mathrm{H}]$ adenosylmethionine & Buckinghamshire \\
\hline Ethanol & Merck, Mannheim & Sodium azide $\left(\mathrm{NaN}_{3}\right)$ & Alfar Aesar, Karlsruhe \\
\hline Ethidium bromide & Roth, Karlsruhe & Sodium chloride $(\mathrm{NaCl})$ & Merck, Mannheim, \\
\hline $\begin{array}{l}\text { Ethylene glycol-bis (b- } \\
\text { aminoethylether)N,N,N',N' } \\
\text { tetraacetic acid (EGTA) }\end{array}$ & Roth, Karlsruhe & Sodium citrate & VWR, Poole \\
\hline $\begin{array}{l}\text { Ethylenediaminetetraacetate } \\
\text { (EDTA) }\end{array}$ & Roth, Karlsruhe & $\begin{array}{l}\text { Sodium dihydrogen } \\
\text { phosphate }\left(\mathrm{NaH}_{2} \mathrm{PO} 4\right)\end{array}$ & Merck, Mannheim \\
\hline Fetal calf serum 10x (FCS) & Sigma, Steinheim & Sodium pyruvate & Gibco, Muenchen \\
\hline Geneticindisulfat (G418) & Roth, Karlsruhe & Sodium sulfate $\left(\mathrm{Na}_{2} \mathrm{SO}_{4}\right)$ & Merck, Mannheim \\
\hline Glucose & Merck, Mannheim & Sodium tetraborate & VWR, Poole \\
\hline Glycerol & Merck, Mannheim & Streptavidin-coated beads & Promega, Madison \\
\hline Glycine & Merck, Mannheim & $\begin{array}{l}\text { Tetramethyl } \\
\text { ethylendiamine (TEMED) }\end{array}$ & Sigma, Steinheim \\
\hline Glycylglycine & $\begin{array}{l}\text { AppliChem } \quad \mathrm{GmbH}, \\
\text { Darmstadt }\end{array}$ & Thiophenol & Sigma, Steinheim \\
\hline Guanidin hydrochlorid & Sigma, Steinheim & Triethanolamine & VWR, Poole \\
\hline Hydrochloric acid ( $\mathrm{HCl})$ & Merck, Mannheim & $\begin{array}{l}\text { Tris (hydroxymethyl)amino } \\
\text { ethane (Tris) }\end{array}$ & Roth, Karlsruhe \\
\hline Hygromycin & Roth, Karlsruhe & Triton X-100 & Merck, Mannheim \\
\hline Isoamylalcohol & Sigma, Steinheim & Tween 20 & Sigma, Steinheim \\
\hline JetPEI & Biomol, Hamburg & Urea & Merck, Mannheim \\
\hline L-Glutamine $100 \mathrm{x}$ & Gibco, Muenchen & Yeast extract & MOBIO, Hamburg \\
\hline Lipofectamine 2000 & Invitrogen, Karlsruhe & & \\
\hline
\end{tabular}

\subsubsection{Buffers and solutions}

Blocking solution: $1 \mathrm{x}$ PBS, $2 \%$ BSA, $0.2 \%$ Triton X100 (v/v), 5\% Normal goat serum $(\mathrm{v} / \mathrm{v})$

Coomassie solution: $2.5 \%$ Coomassie Brilliant Blue, $10 \%$ acetic acid, $50 \%$ methanol

CPD buffer: 20mM Hepes (7.9), 100mM KCl, 10\% Glycerol, 0.1\% Triton, 1mM DTT + 1x Protease Inhibitor

Destaining solution: $10 \%$ acetic acid (v/v), $7.5 \%$ methanol $(\mathrm{v} / \mathrm{v})$

DMEM, complete: 1x DMEM GlutaMAX II [-Pyruvate], 1x LGlutamine, 1x Pencillin Steptomycin, $10 \%$ FCS

DNA loading buffer, 10x: $10 \mathrm{mM}$ EDTA, 30\% w/v glycerol, $100 \mu \mathrm{g} / \mathrm{ml}$ bromphenol blue 
DNase I buffer, 10x: $100 \mathrm{mM}$ Tris- $\mathrm{HCl}(\mathrm{pH} 7.5), 2.5 \mathrm{mM} \mathrm{MgCl} 2,0.5 \mathrm{mM} \mathrm{CaCl}_{2}$

Fixation solution (I): 3\% Paraformaldehyde (w/v) in 1x PBS

Fixation solution (II): $10 \%$ glacial acetic acid (v/v), 30\% methanol (v/v)

Freezing medium: DMEM cell culture medium, 10\% (v/v) DMSO

Laemmli buffer 1x: Tris $63 \mathrm{mM}(\mathrm{pH}$ 6.8), 0.1\% 2-Mercaptoethanol (v/v), 0.0005\%

Bromophenol blue (w/v), 10\% glycerol (v/v), 2\% SDS (w/v)

LB agar: $1.5 \%(\mathrm{w} / \mathrm{v})$ Agar in LB medium

LB Ampicillin medium/agar: LB medium or agar with $100 \mu \mathrm{g} / \mathrm{ml}$ Ampicillin

LB medium: $1 \%(\mathrm{w} / \mathrm{v})$ Peptone, $0.5 \%(\mathrm{w} / \mathrm{v})$ Yeast extract, $0.5 \% \mathrm{NaCl} \%(\mathrm{w} / \mathrm{v}), \mathrm{pH}$ 7,5, autoclaved

Luciferase assay reagent "Firefly": 25 mM Glycylglycine, 15 mM K2 $\mathrm{HPO}_{4}(\mathrm{pH}$ 8.0), 4 mM EGTA, 15 mM MgSO4, 4 mM ATP pH 7.0, 1.25 mM DTT, 0.1 mM Coenzyme A, $80 \mu \mathrm{M}$ Luciferin

Luciferase assay reagent "Renilla": $1.1 \mathrm{M} \mathrm{NaCl}, 2.2 \mathrm{mM} \mathrm{Na} 2 \mathrm{EDTA}, 0.22 \mathrm{M} \mathrm{K}_{2} \mathrm{HPO}_{4}$ (pH 5.1), $0.5 \mathrm{mg} / \mathrm{ml} \mathrm{BSA,} 1.5 \mathrm{mM} \mathrm{NaN}_{3}, 1.5 \mu \mathrm{M}$ Coelenterazine

Mounting medium: Mowiol including $50 \mu \mathrm{g} / \mathrm{ml}$ DAPI

NE buffer A: $10 \mathrm{mM}$ Hepes-KOH (pH 7.9), $1.5 \mathrm{mM} \mathrm{MgCl}, 10 \mathrm{mM} \mathrm{KCl}, 0.34 \mathrm{M}$ Sucrose, 10\% Glycerol (v/v), 1x Protease Inhibitor

NE buffer B: NE Buffer A including 0.2\% NP-40 (v/v)

NM buffer: 50 mM Tris- $\mathrm{HCl}$ (pH 8.0), $50 \mathrm{mM} \mathrm{NaCl}, 10 \mathrm{mM} \mathrm{MgCl} 2,0.5 \mathrm{mM}$ DTT, 10\% sucrose $(\mathrm{w} / \mathrm{v})$, protease inhibitors

PBS 1x: 137 mM NaCl, 2.7 mM KCl, 4.3 mM Na2HPO4 (pH 7.4), 1.47 mM KH $\mathrm{m}_{2} \mathrm{PO}_{4}$

PBST: PBS including $0,1 \%$ of Tween $20(\mathrm{v} / \mathrm{v})$

PD150: 20 mM Hepes $\mathrm{NaOH}$ (pH 7.9), 20\% glycerol, $150 \mathrm{mM} \mathrm{KCl,} \mathrm{0.2 \%} \mathrm{Triton} \mathrm{X100}$ (v/v), 1x Protease Inhibitor

Peptide ligation buffer: $100 \mathrm{mM}$ potassium phosphate ( $\mathrm{pH} 7.9), 3 \mathrm{M}$ guanidine- $\mathrm{HCl}$, $0.5 \%$ benzyl mercaptan (v/v), $0.5 \%$ thiophenol (v/v)

Permibilization solution: $1 \mathrm{xPBS}, 0.2 \%$ Trition X100 (v/v),

Ponceau: $5 \%$ acetic acid (v/v), 0.1\% ponceau $\mathrm{S}(\mathrm{w} / \mathrm{v})$

Precipitation solution: 1\% glycerol (v/v), 10\% PEG 8.000 (w/v) 
Proteinase K buffer: $100 \mathrm{M} \mathrm{NaCl}, 10 \mathrm{mM}$ Tris-HCl (pH 8.0), 25 mM EDTA (pH 8.0), $0.5 \% \mathrm{SDS}, 0.1 \mathrm{mg} / \mathrm{ml}$ proteinase $\mathrm{K}$ (added freshly)

RB high buffer: 10 mM Tris- $\mathrm{HCl}$ (pH 7.5), 1 mM EDTA, $2 \mathrm{M} \mathrm{NaCl}, 1 \mathrm{mM}$ DTT

RB low buffer: $10 \mathrm{mM}$ Tris-HCl (pH 7.5), 1 mM EDTA, $10 \mathrm{mM} \mathrm{NaCl,} 1$ mM DTT

SAU-200 buffer: $7 \mathrm{M}$ deionized urea, $20 \mathrm{mM}$ sodium acetate $(\mathrm{pH}$ 5.2), $1 \mathrm{mM}$ EDTA, 1 $\mathrm{mM}$ DTT, $200 \mathrm{mM} \mathrm{NaCl}$

SB washing buffer: $0.1 \mathrm{M}$ maleic acid, $0.15 \mathrm{M} \mathrm{NaCl}, \mathrm{pH} 7.5,0.3 \%$ Tween 20 (v/v)

SB maleic acid buffer: $0.1 \mathrm{M}$ maleic acid, $0.15 \mathrm{M} \mathrm{NaCl}$, pH 7.5

SB detection buffer: $0.1 \mathrm{M}$ Tris- $\mathrm{HCl}(\mathrm{pH} 9.5), 0.1 \mathrm{M} \mathrm{NaCl}$

SDS-Running buffer: $25 \mathrm{mM}$ Tris, $200 \mathrm{mM}$ glycine. 0.1\% SDS (w/v)

SSC, 20x: $3 \mathrm{M} \mathrm{NaCl}, 0.3 \mathrm{M} \mathrm{Na}_{3}$ citrate (pH 7.0)

TBE: 90 mM Tris, 90 mM Sodiumtetraborate, 2 mM EDTA (pH 8.0)

TEAE buffer: $10 \mathrm{mM}$ triethanolamine- $\mathrm{HCl}(\mathrm{pH} 7.5), 0.1 \mathrm{mM}$ EDTA

Transferbuffer: $200 \mathrm{mM}$ glycine, $25 \mathrm{mM}$ Tris-HCl, 0,04\% SDS (w/v), 20\% Methanol $(\mathrm{v} / \mathrm{v})$

Unfolding buffer: $7 \mathrm{M}$ guanidinium- $\mathrm{HCl}, 20 \mathrm{mM}$ Tris- $\mathrm{HCl}(\mathrm{pH}$ 7.5), 10 mM DTT

Wash buffer: 1x PBS, 0.1\% Triton X100 (v/v)

\subsubsection{General equipment}

Bioruptor

Centrifuge $5415 \mathrm{R}$

Centrifuge $5810 \mathrm{R}$

Cryo Freezing controller

DNA Engine OPTICON real-time PCR

Freezer $-150^{\circ} \mathrm{C}$

Freezer $-80^{\circ} \mathrm{C}$

Gel Doc 2000

Gel dryer Model 583

Hereaus Heracell 240 Incubator
Diogenode, Liége (Belgium)

Eppendorf, Hamburg

Eppendorf, Hamburg

Nalgene, Ohio (USA)

BIO-RAD, Muenchen

Thermo Scientific, Braunschweig

Thermo Scientific, Braunschweig

BIO-RAD, Muenchen

BIO-RAD, Muenchen

Thermo Scientific, Braunschweig 
Hereaus Kelvitron ${ }^{\circledR}$ Incubator

Kodak X OMAT 2000 proccessor

Leica TCS SP5

Microscope Axiovert 40CFL

Mini-PROTEAN 3 Cells

MiniTrans-Blot ${ }^{\circledR}$

Multitron shaker (Bacteria)

Nanodrop ND-1000

Neubauer chamber BRAND,

Orbitrap Xl mass spectrometer

PCR machine epgradient $\mathrm{S}$

PlateChameleon Hidex,

Scanner Perfection V750 PRO

Stuart Gyrorocker SSL3

Sub-Cell-GT® Agarose gel electrophoresis BIO-RAD, Muenchen

Thermomixer comfort
Thermo Scientific, Braunschweig

Carestream Health, New York (USA)

Leica, Wetzlar

Zeiss, Jena

BIO-RAD, Muenchen

BIO-RAD, Muenchen

HT Infors, Braunschweig

Peqlab, Erlangen

Wertheim

Thermo Scientific, Braunschweig

Eppendorf, Hamburg

Turku (Finnland)

Epson, Meerbusch

Sigma, Steinheim

Eppendorf, Hamburg

\subsubsection{Antibodies}

\begin{tabular}{lllll}
\hline Antibody & Host & Supplier & Application & Dilution \\
\hline$\alpha$ ADNP & Mouse, mc & BD Biosciences & IF & $1: 70$ \\
& & & WB & $1: 1.000$ \\
$\alpha \beta$-tubulin & Mouse, mc & Sigma & WB & $1: 20.000$ \\
$\alpha$ CDYL & Rabbit, pc & Abcam & WB & $1: 1.000$ \\
$\alpha$ Digoxigenin-AP & Mouse, mc & Roche & SB & $1: 1.000$ \\
$\alpha$ FLAG-M2 & Mouse, mc & Sigma & WB & $1: 1.000$ \\
$\alpha$ GFP & Mouse, mc & Roche & IP & $5 \mu 1 /$ sample \\
\hline$\alpha H 3$ & Rabbit, pc & Abcam & WB & $1: 25.000$ \\
$\alpha H 3$ 27me1 & Rabbit, pc & Thomas Jenuwein & IF & $1: 500$ \\
& & & WB & $1: 1.000$ \\
$\alpha H 3$ K5me3 & Rabbit, pc & Upstate/Millipore & IF & $1: 500$ \\
& & & WB & $1: 1.000$
\end{tabular}


CHAPTER 2 - Material and Methods

\begin{tabular}{|c|c|c|c|c|}
\hline Antibody & Host & Supplier & Application & Dilution \\
\hline$\alpha \mathrm{H} 3 \mathrm{~K} 9 \mathrm{me} 3 \mathrm{~S} 10 \mathrm{ph}$ & Rabbit, pc & Upstate/Millipore & WB & $1: 1.000$ \\
\hline$\alpha \mathrm{H} 3 \mathrm{~K} 9 \mathrm{ac}$ & Rabbit, pc & Upstate/Millipore & WB & $1: 1.000$ \\
\hline$\alpha \mathrm{H} 4$ & Rabbit, pc & Abcam & WB & $1: 1.000$ \\
\hline \multirow[t]{2}{*}{$\alpha \mathrm{H} 4 \mathrm{~K} 20 \mathrm{me} 3$} & Rabbit, pc & Upstate/Millipore & IF & $1: 500$ \\
\hline & & & WB & $1: 1.000$ \\
\hline \multirow[t]{2}{*}{$\alpha H P 1 \alpha$} & Mouse, mc & Chemicon/Millipore & IF (MAB3584) & $1: 2.500$ \\
\hline & & & WB (MAB3446) & $1: 1.000$ \\
\hline \multirow[t]{2}{*}{$\alpha \mathrm{HP} 1 \beta$} & Mouse, mc & Chemicon/Millipore & IF & $1: 7.500$ \\
\hline & & & WB & $1: 10.000$ \\
\hline \multirow[t]{2}{*}{$\alpha \mathrm{HP} 1 \gamma$} & Mouse, mc & Chemicon/Millipore & IF & $1: 15.000$ \\
\hline & & & WB & $1: 20.000$ \\
\hline amouse-IgG-Alexa 555 & Goat & Molecular Probes & IF & $1: 1.000$ \\
\hline amouse-IgG -HRP & Goat & DakoCytomation & WB & $1: 3.000$ \\
\hline amouse-IgG magnetic & Sheep & DYNAL Biotech & IP & $40 \mu 1$ / sample \\
\hline \multicolumn{5}{|l|}{ beads } \\
\hline arabbit-IgG -Alexa 488 & Donkey & Molecular Probes & IF & $1: 1.000$ \\
\hline arabbit-IgG -HRP & Swine & DakoCytomation & WB & $1: 3.000$ \\
\hline
\end{tabular}

Table 2-1: Antibodies

List of antibodies, suppliers, the application for which they are used and the dilutions. mc: monoclonal; pc: polyclonal; Applications: IF: Immunofluorescence stainung; IP: Immunoprecipitation; WB: Western Blot analysis; SB: Southern blot analysis 


\subsection{Molecular biological methods}

\subsubsection{Agarose gel electrophoresis}

Agarose gel electrophoresis of DNA was carried out according to (Sambrook and Russel, 2001). Typically, $0.5 \%$ to $2.0 \%$ agarose gels (w/v agarose in TBE) were used. Gels were poured and run using the Sub-Cell GT horizontal electrophoresis system (Bio-Rad). Samples were mixed with 10x DNA loading buffer and run on agarose gels for 45 min at a constant voltage of $110 \mathrm{~V}$ in TBE buffer at RT. $1 \mathrm{~kb}$ Plus DNA Ladder (Fermentas) was used as size reference. DNA was visualized by staining of the agarose gels after electrophoresis with $1 \mu \mathrm{g} / \mathrm{ml}$ ethidium bromide for $20 \mathrm{~min}$ at RT.

\subsubsection{Plasmid manipulation}

Constructs were generated by standard techiques of DNA cloning (Sambrook and Russel, 2001). Restriction endonucleases and ligase from NEB and PfuII Ultra polymerase (Stratagene) were used according to the manufacturers instructions.

ADNP was amplified by PCR from an EST (expressed sequence tag) clone (I.M.A.G.E. Consortium Clone ID 3448801). PCR products were then cloned into the destination vectors via the restriction sites given in (Table 2-2).

Site directed mutagenesis was performed by fusion PCR. In a first step two fragments of ADNP were generated by PCR using either the forward or the reverse primers for cloning and overlapping mutagenesis primers containing the point mutation. These fragments were fused by PCR using both fragments as template and the cloning primers.

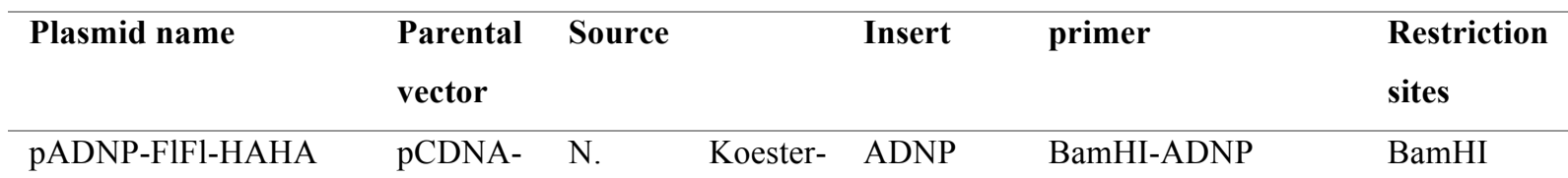


CHAPTER 2 - Material and Methods

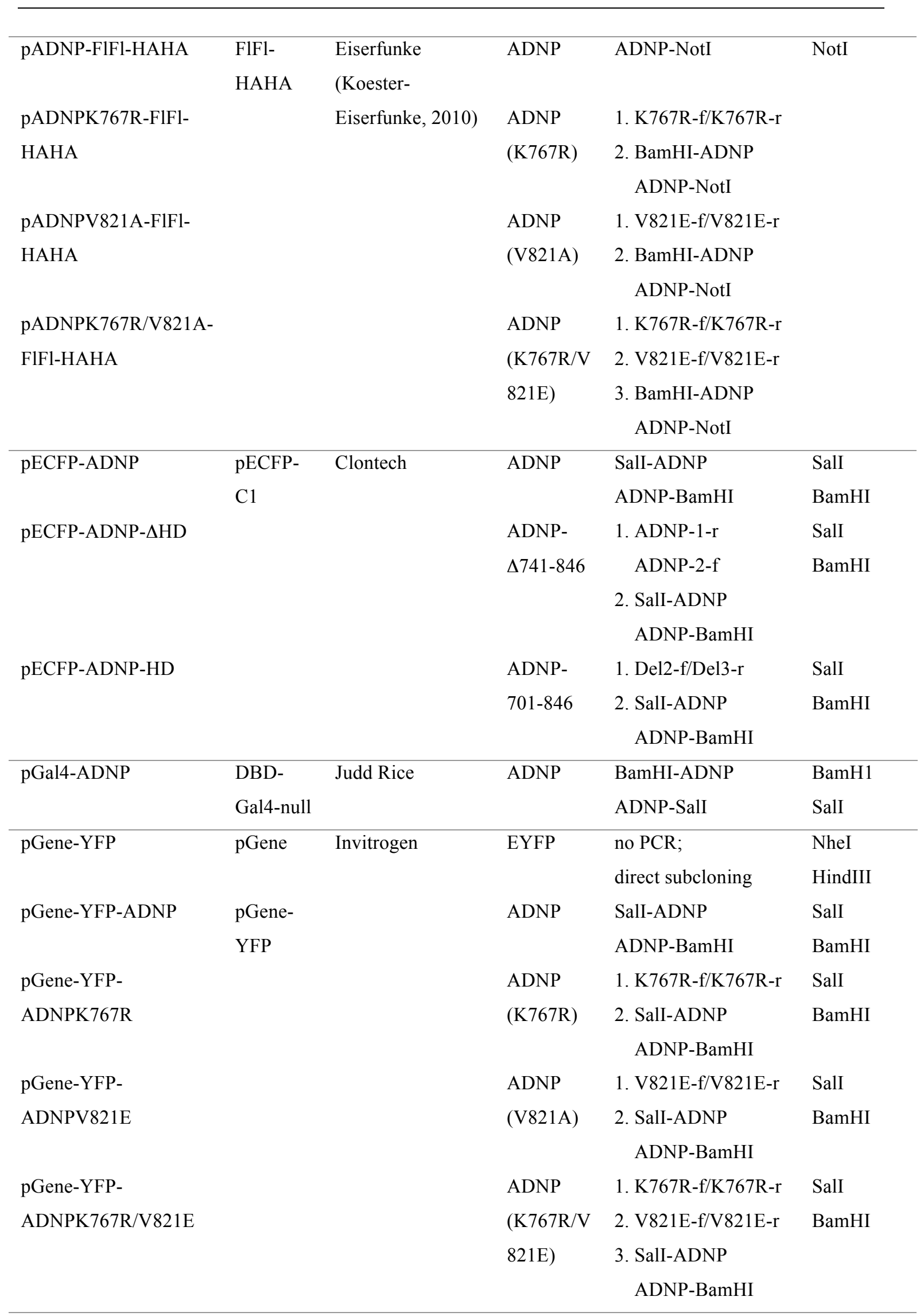


Table 2-2: Cloning details

For vector information see

pCDNA-FlFl-HAHA: (Koester-Eiserfunke, 2010)

pECFP-C1: $\quad$ www.clontech.com/images/pt/dis_vectors/PT3259-5.pdf

DBD-Gal4-null: (Kalakonda et al., 2008)

pGene: toolszh.invitrogen.com/content/sfs/vectors/pgenev5his_map.pdf

Primers:

ADNP-1-r: 5'-CAGCCACTCAGCATCAAAATCAAAGCAGCTAGGGGAATCAGC-3'

ADNP-2-f: 5 '-GCTGATTCCCCTAGCTGCTTTGATTTTGATGCTGAGTGGCTG-3'

ADNP-BamHI: 5'-TCCGGTGGATCCCTAGGCTTGCTGGCTGCTC-3'

ADNP-NotI: 5'-GTATGCGGCCGCGGCTTGCTGGCTGCTCAGC-3'

ADNP-SalI: 5'-GCTCGAGTCGACGCTAGGCTTGCTGGCTGCTCAGC-3'

BamHI-ADNP: 5'-GAATTGGGATCCATGTTCCAACTTCCTGTCAAC-3'

Del2-f: 5'-TGGTACGTCGACGCACCTTCTCGGCTCAATC-3'

Del3-r: 5'-TCCGGTGGATCCCTATTCAAACAGCCACTCAGCATC-3'

K767R-f: $\quad$ 5'-CTTATGAGGCTAGGAGAAGCTTTCTCAC-3'

K767R-r: 5'-GTGAGAAAGCTTCTCCTAGCCTCATAAG-3'

Sall-ADNP: 5'-TGGTACGTCGACATGTTCCAACTTCCTGTCAAC-3'

V821E-f: $\quad$ 5'-GTACAAGCCTGGTGAGCTGCTAGGTTTTAAC-3'

V821E-r: $\quad$ '-ACCTAGCAGCTCACCAGGCTTGTACTTTTCACAG-3'

Insert sequences:

The EYFP was subcloned from pEYFP-C1 (Clontech):

ATGGTGAGCAAGGGCGAGGAGCTGTTCACCGGGGTGGTGCCCATCCTGGTCGAGCTGGACGG CGACGTAAACGGCCACAAGTTCAGCGTGTCCGGCGAGGGCGAGGGCGATGCCACCTACGGCA AGCTGACCCTGAAGTTCATCTGCACCACCGGCAAGCTGCCCGTGCCCTGGCCCACCCTCGTGA CCACCTTCGGCTACGGCCTGCAGTGCTTCGCCCGCTACCCCGACCACATGAAGCAGCACGACT TCTTCAAGTCCGCCATGCCCGAAGGCTACGTCCAGGAGCGCACCATCTTCTTCAAGGACGACG GCAACTACAAGACCCGCGCCGAGGTGAAGTTCGAGGGCGACACCCTGGTGAACCGCATCGAG CTGAAGGGCATCGACTTCAAGGAGGACGGCAACATCCTGGGGCACAAGCTGGAGTACAACTA CAACAGCCACAACGTCTATATCATGGCCGACAAGCAGAAGAACGGCATCAAGGTGAACTTCA AGATCCGCCACAACATCGAGGACGGCAGCGTGCAGCTCGCCGACCACTACCAGCAGAACACC CCCATCGGCGACGGCCCCGTGCTGCTGCCCGACAACCACTACCTGAGCTACCAGTCCGCCCTG 
AGCAAAGACCCCAACGAGAAGCGCGATCACATGGTCCTGCTGGAGTTCGTGACCGCCGCCGG GATCACTCTCGGCATGGACGAGCTGTACA

The ADNP sequence was derived fron the I.M.A.G.E. Consortium Clone ID 3448801:

ATGTTCCAACTTCCTGTCAACAATCTTGGCAGTTTAAGAAAAGCCCGGAAAACTGTGAAAAAA ATACTTAGTGACATTGGGTTGGAATACTGTAAAGAACATATAGAAGATTTTAAACAGTTTGAA CCTAATGACTTTTATTTGAAAAACACTACATGGGAGGATGTAGGACTGTGGGACCCTTCTCTT ACGAAAAATCAGGACTATCGGACAAAACCTTTTTGCTGCAGTGCTTGTCCGTTTTCCTCAAAA TTCTTCTCTGCCTACAAAAGTCATTTCCGGAATGTCCATAGTGAAGACTTTGAAAATAGGATTC TCCTTAACTGCCCTTACTGTACCTTCAATGCAGATAAAAAGACTTTGGAAACACACATTAAAA TATTTCATGCTCCAAACTCCGGCGCACCAAGTAGCAGCCTCAGCACTTTCAAAGATAAAAACA AAAACGATGGCCTTAAACCTAAGCAGGCTGACAATGTAGAGCAAGCCGTGTATTACTGCAAG AAGTGCACTTACCGAGACCCTCTCTACGAGATCGTCAGGAAGCACATCTACAGGGAACATTTT CAACACGTGGCAGCACCCTACATAGCAAAAGCAGGAGAAAAATCACTCAATGGTGCAGTCTC CCTGGGCACAAATGCCCGAGAGGAGTGTAACATCCACTGCAAGCGATGCCTTTTCATGCCCAA GTCCTATGAAGCTTTGGTACAGCATGTCATTGAGGACCATGAACGGATAGGCTATCAGGTCAC TGCCATGATCGGACACACAAATGTTGTAGTTCCCCGAGCCAAGCCCTTGATGCTGATAGCTCC CAAACCTCAAGACAAAAAGGGCATGGGACTCCCACCACGAATCAGCTCCCTTGCTTCTGGAA ATGTCCGGTCGTTGCCATCACAGCAGATGGTAAACCGATTGTCAATACCAAAGCCCAACTCAA ATTCAACGGGAGTCAACATGATGTCCAATGTTCACCTGCAGCAAAACAACTATGGAGTCAAAT CTGTGGGCCAGAGCTATGGTGTTGGCCAGTCAGTGAGGCTGGGACTAGGTGGCAATGCTCCA GTTTCCATCCCTCAACAGTCTCAGTCCGTGAAACAGTTACTTCCAAGTGGGAATGGGAGGTCT TTTGGGCTAGGTGCTGAGCAGAGGCCCCCAGCAGCAGCCAGGTACTCCCTGCAGACTGCCAA CACCTCTCTACCCCCAGGCCAAGTGAAGTCTCCCTCTGTGTCTCAGTCACAGGCATCTAGAGT ATTAGGTCAGTCCAGTTCTAAACCTCCACCAGCCGCCACAGGCCCTCCTCCAAGCAACCACTG TGCCACTCAGAAGTGGAAAATCTGTACAATCTGTAACGAGCTTATCCCTGAGAATGTCTATAG CGTTCACTTCGAAAAGGAGCATAAAGCTGAGAAAGTCCCAGCCGTAGCTAACTACATTATGA AAATACACAATTTTACTAGCAAATGCCTCTACTGTAATCGCTATTTGCCTACAGATACCCTACT CAACCATATGTTAATTCATGGTCTGTCTTGTCCGTATTGCCGTTCCACCTTCAATGATGTAGAG AAGATGGCAGCACACATGCGAATGGTTCATATTGATGAAGAGATGGGGCCTAAAACGGATTC TACTTTGAGCTTTGATTTGACATTGCAACAGGGCAGTCACACCAACATTCATCTCCTGGTGACC ACATACAACCTGAGGGATGCCCCGGCTGAATCAGTTGCTTACCATGCCCAAAATAATGCCCCA GTTCCTCCAAAGCCACAACCAAAAGTTCAGGAAAAAGCAGATGTCCCGGTTAAAAGTTCACC TCAAGCTGCAGTGCCCTATAAAAAAGATGTTGGGAAGACCCTTTGCCCTCTTTGCTTTTCAATA CTAAAAGGACCCATATCTGATGCACTTGCACATCATTTACGAGAAAGACACCAAGTTATTCAG ACAGTTCATCCGGTTGAGAAAAAGCTAACTTACAAATGTATCCATTGCCTTGGTGTGTATACT 
AGCAACATGACAGCCTCAACCATCACTCTGCATCTAGTCCACTGCAGGGGTGTTGGAAAAACC CAGAATGGCCAGGACAAGACAAACGCACCTTCTCGGCTCAATCAGTCTCCAGGCCTGGCCCCT GTGAAGCGCACGTATGAGCAGATGGAGTTTCCACTGCTAAAAAAGCGGAAGCTGGAGGAGGA TGCTGATTCCCCTAGCTGCTTTGAAGAGAAGCCAGAAGAGCCTGTTGTTTTAGCTTTAGACCC CAAGGGTCATGAAGATGATTCTTATGAGGCTAGGAAAAGCTTTCTCACAAAGTACTTCAACAA ACAGCCCTATCCCACCAGGAGAGAAATTGAGAAGTTAGCTGCCAGTCTATGGCTATGGAAGA GTGACATTGCCTCCCATTTCAGTAACAAGAGGAAGAAGTGTGTCCGCGACTGTGAAAAGTAC AAGCCTGGTGTGCTGCTAGGTTTTAACATGAAAGAATTAAATAAAGTCAAACACGAGATGGA TTTTGATGCTGAGTGGCTGTTTGAAAATCACGATGAGAAAGACTCAAGAGTCAATGCTAGCAA GACTGTTGACAAAAAGCATAACCTTGGGAAAGAAGATGATAGCTTCTCAGATAGTTTTGAAC ATTTGGAAGAAGAATCCAATGGAAGCGGGAGTCCTTTTGACCCTGTCTTTGAAGTTGAGCCTA AAATTCCCAGTGATAATTTAGAGGAGCCTGTACCGAAGGTTATTCCGGAAGGTGCTTTGGAAT CTGAGAAGCTAGACCAAAAAGAGGAGGAGGAGGAGGAGGAGGAGGAGGATGGTTCAAAATA TGAAACTATCCATTTGACTGAGGAACCAGCCAAATTAATGCATGATGCCTCTGATAGTGAGGT AGACCAAGATGATGTAGTTGAGTGGAAAGATGGTGCTTCACCATCTGAGAGTGGGCCTGGTTC CCAACAAATCTCAGACTTTGAGGATAATACATGTGAAATGAAACCAGGAACCTGGTCTGATG AGTCTTCCCAGAGTGAAGATGCAAGGAGCAGTAAGCCAGCTGCCAAAAAAAAGGCTACAGTG CAAGATGACACAGAGCAGTTAAAATGGAAGAATAGTTCCTATGGAAAAGTTGAAGGGTTTTG GTCCAAGGACCAGTCACAGTGGGAAAATGCATCTGAGAATGCAGAGCGCTTACCAAACCCAC AGATTGAGTGGCAGAATAGCACAATTGACAGTGAGGACGGGGAGCAGTTTGACAGCATGACT GACGGAGTTGCTGATCCCATGCATGGCAGCTTAACTGGAGTGAAGCTGAGCAGCCAGCAAGC $\mathrm{C}$

Labeled in red are the two bases $A$, which are mutated to $G$ to achieve the K767R mutation and to $\mathrm{T}$ for the V821E mutation respectively.

\subsection{3 cloning}

To $100 \mu \mathrm{l}$ chemically competent E.coli DH5 $\sim 100 \mathrm{ng}$ transforming DNA was added. The tubes containing these cells were stored for on ice $20 \mathrm{~min}$, transferred for $1 \mathrm{~min}$ into a preheated $42^{\circ} \mathrm{C}$ waterbath and rapidly cooled on ice for $2 \mathrm{~min}$. Thereafter the transformation-mix was suspended in prewarmed $37^{\circ} \mathrm{C} \mathrm{LB}$-medium and the cells were allowed to recover for $45 \mathrm{~min}$ at $37^{\circ} \mathrm{C}$ and $1000 \mathrm{rpm}$ in a thermomixer. $100 \mu 1$ of the cell suspension were plated on agar-plates, containing 33,34 $\mu \mathrm{g} / \mathrm{ml}$ kanamycin or $100 \mu \mathrm{g} / \mathrm{ml}$ ampicillin respectively and grown over night at $37^{\circ} \mathrm{C}$. Single colonies were picked into 5 $\mathrm{ml} \mathrm{LB}$ medium containing $50 \mu \mathrm{g} / \mathrm{ml}$ kanamycin or $100 \mu \mathrm{g} / \mathrm{ml}$ ampicillin respectively and 
incubated at $37^{\circ} \mathrm{C}$ overnight. Plasmids were purified using a MiniPrep Kit (Qiagen, Hilden). To screen for positive colonies, $10 \%$ of the purified plasmids were digested with the cloning restriction enzymes and separated by electrophoresis on a $1 \%$ agarose gel in TBE buffer. Plasmids showing the right insert size were sequenced by MWG (Ebersberg) or Seqlab (Goettingen).

\subsubsection{Preparation of genomic DNA}

Genomic DNA was prepared from cell aliquots (2.3.5) as described (Ausubel et al., 1998). Briefly, cell pellets were resuspended in Proteinase K buffer and digested over night at $50^{\circ} \mathrm{C}$. The samples were extracted with an equal volume of phenol/chloroform/isoamylalcohol by centrifugation for $10 \mathrm{~min}$ at $1.700 \mathrm{xg}$. The aqueous (top) layer was transferred into a new tube. DNA was precipitated with $1 / 2$ vol of $7.5 \mathrm{M}$ ammonium sulfate and $2 \mathrm{vol}$ of $100 \%$ Ethanol. After centrifugation for 2 min at $1.700 \mathrm{xg}$ and one washing step with $70 \%$ Ethanol the pellet was dried and resuspended in $10 \mathrm{mM}$ Tris- $\mathrm{HCl}(\mathrm{pH} 8.0)$ and stored at $-20^{\circ} \mathrm{C}$.

\subsubsection{Southern blot analysis}

The DNA prepared in 2.2.4 was digested with TaiI (NEB) according to the manufacturers instructions. The samples were separated on a $1 \%$ agarose gel and stained as described in 2.2.1. Southern blot was performed according to (Ausubel et al., 1998) with modifications: The gel was rinsed in $\mathrm{H}_{2} \mathrm{O}$, depurinated for 20 min in $0.25 \mathrm{M} \mathrm{HCl}$ and neutralized for $30 \mathrm{~min}$ in $0.4 \mathrm{M} \mathrm{NaOH}$. Downward transfer of the DNA onto a positively charged nylon transfer membrane (GE Healthcare) was performed over night using $0.4 \mathrm{M}$ $\mathrm{NaOH}$ as transfer buffer. Major satellite DNA was detected using the DIG High Prime DNA Labeling and Detection Starter Kit I (Roche Diagnostics GmbH, Mannheim) as described by the manufacturer. Briefly, the major satellite probe was generated by PCR using DIG-labeled dUTP : dTTP 1:3, genomic DNA of NIH 3T3 cells as template and major satellite repeat primers (5'-ATATGTTGAGAAAACTGAAAATCACG-3'; 5'- 
CCTTCAGTGTGCATTTCTCATTTTTCAC-3'). After washing the membrane twice in 2x SSC for $10 \mathrm{~min}$ at RT it was prehybridized in DIG Easy Hyb for 30 min at $37^{\circ} \mathrm{C} .25$ $\mathrm{ng} / \mathrm{ml}$ DIG-labeled DNA probe was denatured by boiling for $5 \mathrm{~min}$ and rapidly cooling in ice water. The probe was mixed with prewarmed DIG Easy Hyb and hybridized over night at $42^{\circ} \mathrm{C}$. Two post-hybridization washes for $5 \mathrm{~min}$ in $2 \mathrm{x} \mathrm{SSC}, 0.1 \% \mathrm{SDS}$ at RT were followed by 2 washes in $0.5 x \mathrm{SSC}, 0.1 \% \mathrm{SDS}$ at $65-68^{\circ} \mathrm{C}$. The membrane was rinsed in SB washing buffer, blocked in 1x Blocking solution in SB washing buffer for 30 min and incubated with Anti-Digoxigenin-AP antibody in Blocking solution in SB washing buffer for another $30 \mathrm{~min}$ at RT. Thereafter, the membrane was washed twice in SB washing buffer for $15 \mathrm{~min}$ at RT and equilibrated for $2-5 \mathrm{~min}$ in SB detection buffer. For detection, $200 \mu \mathrm{l}$ of NBT/BCIP stock solution were added to $10 \mathrm{ml}$ of SB detection buffer and applied to the membrane. The color reaction was performed in the dark until bands became visible and then stopped by washing the membrane in $\mathrm{H}_{2} \mathrm{O}$ for 5 $\min$.

\subsubsection{RNA isolation}

Cell aliquots prepared in 2.3.5 were resuspended in $1 \mathrm{ml}$ TRIzol ${ }^{\circledR}$ reagent. $0.2 \mathrm{ml}$ chloroform were added and mixed vigorously for $15 \mathrm{~s}$. After 2-3 min incubation at RT the samples were centrifuged for $15 \mathrm{~min}$ at $12.000 \mathrm{xg}, 4^{\circ} \mathrm{C}$. The upper aqueous phase was transferred into a new tube, mixed with $0.5 \mathrm{ml}$ isopropanol and incubated for $10 \mathrm{~min}$ at RT. Thereafter the RNA precipitate was centrifuged for $10 \mathrm{~min}$ at $12.000 \mathrm{xg}, 4^{\circ} \mathrm{C}$. The pellet was washed in $75 \%$ Ethanol and centrifuged for $10 \mathrm{~min}$ at $7.600 \mathrm{xg}, 4^{\circ} \mathrm{C}$. Then the pellet was dried and redissolved in $100 \mu \mathrm{l}$ RNase-free water. The concentration was measured using the Nanodrop ND 1000.

\subsection{7 cDNA synthesis}

First strand synthesis of cDNA was performed using the Superscript II Kit (Invitrogen, Karlsbad) according to the manufacturers protocol. Before cDNA synthesis 
contaminating gemomic DNA was removed from the RNA by digesting DNAse I mix for $30 \mathrm{~min}$ at $37^{\circ} \mathrm{C}$. The reaction was stopped by adding $0.4 \mu 1100 \mathrm{mM}$ EDTA and DNAse I inactivation for $10 \mathrm{~min}$ at $75^{\circ} \mathrm{C}$. $1 \mu \mathrm{l}$ random hexamers and dNTPs were added and incubated for $5 \mathrm{~min}$ at $65^{\circ} \mathrm{C}$. After rapid cooling the samples in ice water the Superscript II mix was added. Reverse transcription was performed at $25^{\circ} \mathrm{C}$ for 10 min followed by a $50^{\circ} \mathrm{C}$ incubation for $10 \mathrm{~min}$ and terminated for $5 \mathrm{~min}$ at $85^{\circ} \mathrm{C}$. RNA was digested by addition of $1 \mu \mathrm{RNAse} \mathrm{H}$ and incubation at $37^{\circ} \mathrm{C}$ for $20 \mathrm{~min}$. The cDNA was stored at $20^{\circ} \mathrm{C}$ for further use.

\section{DNAse I mix:}

RNA:

DNAse I buffer:

DNAse I (NEB):

RNAse OUT:

H2O (RNAse-free):
$3 \mu \mathrm{g}$

$$
0.8 \mu 1
$$

$0.8 \mu 1$

$1 \mu 1$ to $8 \mu 1$

\section{Superscript II mix:}

RT buffer: $\quad 2 \mu 1$

$\mathrm{MgCl2:} \quad 4 \mu \mathrm{l}$

0.1 M DTT: $\quad 2 \mu 1$

RNAse OUT: $\quad 1 \mu \mathrm{l}$

Superscript II: $\quad 1 \mu \mathrm{l}$

\subsubsection{Real time PCR}

To quantify the relative amount of major satellite repeat transcripts real time PCR was performed. All PCR were done in triplicate. $2 \mu$ of 1:4 diluted cDNA (2.2.7) were added to $18 \mu 1$ master-mix $\left(1.25 \mu \mathrm{M}\right.$ of each primer, $10 \mu 12 \mathrm{x}$ iQ SYBR ${ }^{\circ}$ Green Supermix (BIO-RAD, Muenchen), $\mathrm{H}_{2} \mathrm{O}$ to $18 \mu \mathrm{l}$ ) and processed in a DNA Engine OPTICON realtime PCR machine with the following program: $15 \mathrm{~min}$ at $95^{\circ} \mathrm{C}-\left[15 \mathrm{~s}\right.$ at $94^{\circ} \mathrm{C}-55 \mathrm{~s}$ at $55^{\circ} \mathrm{C}-30 \mathrm{~s}$ at $72^{\circ} \mathrm{C}$ - read plate $]_{40 \mathrm{x}}$ - perform melting curve from $60^{\circ} \mathrm{C}$ to $95^{\circ} \mathrm{C}$, read every $0.3^{\circ} \mathrm{C}$. To calculate the relative expression levels, the major satellite signals were normalized to the signals of GAPDH as follows: $\mathrm{Ct}_{\text {major satellite repeat }}-\mathrm{Ct}_{\mathrm{GAPDH}}=\Delta \mathrm{Ct}$. The average of the $2^{-{ }_{\Lambda} \mathrm{Ct}}$ values from the triplicates were blotted with the standard deviation of the triplicate as error bars. 
Primers:

Major satellite repeats:

Fwd: 5'-ATATGTTGAGAAAACTGAAAATCACG-3'

Rev: 5'-CCTTCAGTGTGCATTTCTCATTTTTCAC-3'

GAPDH:

Fwd: 5'-AGGTCGGTGTGAACGGATTTG-3'

Rev: 5'-TGTAGACCATGTAGTTGAGGTCA-3'

\subsection{Mammalian cell culture}

\begin{tabular}{|c|c|c|c|}
\hline Cell line & Origin & Medium & Supplier \\
\hline HEK 293 & $\begin{array}{l}\text { Human, embryonic } \\
\text { kidney }\end{array}$ & DMEM, complete & Wolfgang Fischle \\
\hline HEK TK22 & $\begin{array}{l}\text { Human, embryonic } \\
\text { kidney }\end{array}$ & $\begin{array}{l}\text { DMEM, complete } \\
+5 \mu \mathrm{g} / \mathrm{ml} \text { Puromycin }\end{array}$ & Judd Rice \\
\hline $\begin{array}{l}\text { MEF (isogenic } \\
\text { to } \quad \text { MEF } \\
\text { Suv39h1/h2 -/-) }\end{array}$ & $\begin{array}{l}\text { Mouse, embryonic } \\
\text { fibroblast }\end{array}$ & $\begin{array}{l}\text { DMEM, complete } \\
+1 \mathrm{x} \text { non essential } \\
\text { amino acids, } 1 \mathrm{x} \\
\text { sodium pyruvate }\end{array}$ & Thomas Jenuwein \\
\hline $\begin{array}{l}\text { MEF } \\
\text { Suv39h1/h2 -/- }\end{array}$ & $\begin{array}{l}\text { Mouse, embryonic } \\
\text { fibroblast }\end{array}$ & $\begin{array}{l}\text { DMEM, complete } \\
+1 \mathrm{x} \text { non essential } \\
\text { amino acids, } 1 \mathrm{x} \\
\text { sodium pyruvate }\end{array}$ & Thomas Jenuwein \\
\hline $\begin{array}{l}\text { MEF (isogenic } \\
\text { to MEF HP1 } \alpha- \\
/-; \quad \text { HP1 } \beta \\
\text { HP1 } \gamma \quad-/-; \\
\text { HP1 } \alpha / \beta-/-)\end{array}$ & $\begin{array}{l}\text { Mouse, embryonic } \\
\text { fibroblast }\end{array}$ & $\begin{array}{l}\text { DMEM, complete } \\
+1 \mathrm{x} \text { non essential } \\
\text { amino acids, } 1 \mathrm{x} \\
\text { sodium pyruvate }\end{array}$ & Prim Singh \\
\hline $\begin{array}{l}\text { MEF HP1 } \alpha-/-; \\
\text { HP1 } \beta \text {-/-; HP1 } \gamma \\
-/-; \mathrm{HP} 1 \alpha / \beta-/-\end{array}$ & $\begin{array}{l}\text { Mouse, embryonic } \\
\text { fibroblast }\end{array}$ & $\begin{array}{l}\text { DMEM, complete } \\
+1 \mathrm{x} \text { non essential } \\
\text { amino acids, } 1 \mathrm{x} \\
\text { sodium pyruvate }\end{array}$ & Prim Singh \\
\hline NIH $3 \mathrm{~T} 3$ & Mouse, fibroblast & DMEM, complete & DMSZ \\
\hline $\begin{array}{l}\text { GeneSwitch } \\
\text { NIH 3T3 }\end{array}$ & Mouse, fibroblast & $\begin{array}{l}\text { DMEM, complete } \\
+\quad 100 \quad \mu \mathrm{g} / \mathrm{ml} \\
\text { Hygromycin }\end{array}$ & Invitrogen \\
\hline
\end{tabular}

Table 2-3: Cell lines and media 


\subsubsection{Thawing}

To thaw cells from frozen stocks $10 \mathrm{ml}$ of the appropriate medium was equilibrated to $37^{\circ} \mathrm{C}$ and $5 \% \mathrm{CO}_{2}$. The cells were thawed in a $37^{\circ} \mathrm{C}$ water bath, resuspended with $1 \mathrm{ml}$ prewarmed medium (Table 2-3), added dropwise to the equilibrated medium and grown at $37^{\circ} \mathrm{C}$ and $5 \% \mathrm{CO}_{2}$ until they reached $\sim 80 \%$ confluency.

\subsubsection{Maintenance}

The cell lines were grown in the medium given in Table $2-3$ at $37^{\circ} \mathrm{C}$ and $5 \% \mathrm{CO}_{2}$. At $90 \%$ confluency cells were washed with prewarmed PBS and trypsinized for $10 \mathrm{~min}$ in $0.05 \%$ Trypsin-EDTA. Supplemented medium was added to stop the reaction. The cells were subsequently plated in 1:10 (wild type) or 1:5 (knockout cell-lines) dilution to be $90 \%$ confluent after three days (NIH3T3, HEK293, GeneSwitchNIH3T3) or five days (wild type and knockout MEF cells).

\subsubsection{Freezing}

The cells at $70 \%$ confluency were washed with PBS and trypsinized as described above. The number of viable cells was determined by mixing of the cells with one volume $0.4 \%$ Trypan blue and counting in a Neubauer chamber. After centrifugation for $5 \mathrm{~min}$ at $250 \mathrm{xg}$ and room temperature the cells were resuspended in $4^{\circ} \mathrm{C}$ freezing medium (culture

medium with $5 \%$ DMSO) in an appropriate volume to obtain $1 * 10^{6}$ cells $/ \mathrm{ml} .1 \mathrm{ml}$ cell suspension was aliquoted per cryotube on ice. These tubes were stored in a precooled Cryo Freezing Controller at $-80^{\circ} \mathrm{C}$ for $48 \mathrm{~h}$ to allow a cooling by approximately $1^{\circ} \mathrm{C}$ per $\min$. Thereafter the cells were transferred to $-150^{\circ} \mathrm{C}$ for long time storage. 


\subsubsection{Generation of stable cell lines}

One day prior to transfection GeneSwitchNIH3T3 cells were seeded in $10 \mathrm{~cm}$ dishes to reach $\sim 50 \%$ confluency at the day of transfection. Transfection was performed using JetPEI reagent (Biomol, Hamburg) in accordance with the instructions of the manufacturer. Typically $16 \mu$ JetPEI reagent and $8 \mu \mathrm{g}$ of pGeneYFP, pGeneYFP-ADNP, pGeneYFP-ADNP(K767R), pGeneYFP-ADNP(V821E) and pGeneYFP$\operatorname{ADNP}(\mathrm{K} 767 \mathrm{R} / \mathrm{V} 821 \mathrm{E})$ were used per dish. One day after transfection, the cells were trypsinized and transferred to $15 \mathrm{~cm}$ dishes containing medium supplemented with 400 $\mu \mathrm{g} / \mathrm{ml}$ zeocin (Invitrogen). To remove dead cells the medium was exchanged after two days. Untransfected cells were treated at the side as a control to verify that the treatment was working. Two weeks post transfection separate colonies were visible. Expression of YFP/YFP-ADNP was induced with $10 \mathrm{ng} / \mathrm{ml}$ Mifepristone. The cells were washed with PBS and covered with $37^{\circ} \mathrm{C}$ warm $0.5 \%$ low melting agarose in PBS. After the agarose polymerized at room temperature YFP/YFP-ADNP expressing cells were picked using

cloning cylinders and transferred into 24 well plates. Stable inducible colonies were analyzed by fluorescence microscopy (Axiovert $40 \mathrm{CFL}$ ) and Western Blot. Two colonies of moderately overexpressing cell-lines for each construct were frozen for long term storage.

\subsubsection{Transfection of siRNA}

NIH3T3 cells were seeded in 6-well plates one day prior to transfection to be $30-50 \%$ confluent at the day of transfection. $100 \mu \mathrm{g}$ siRNA was transfected with $5 \mu \mathrm{l}$ Lipofectamine2000 as described by the manufacturer (Invitrogen, Karlsruhe). One day post transfection the cells were trypsinized and transferred to a $10 \mathrm{~cm}$ dish where the transfection was repeated with $600 \mu \mathrm{g}$ siRNA and $30 \mu \mathrm{l}$ Lipofectamine2000. The next day the cells were washed with ice cold PBS, scraped off the plates and aliquoted into 3 samples for protein, DNA and RNA extraction. 


\subsection{Cell based assays}

\subsubsection{Dual luciferase assay}

HEK293 and HEK293 TK22 (Kalakonda et al., 2008) cells were seeded at a density of $1.5 \times 10^{5}$ cells/well into 12 -well plates $24 \mathrm{~h}$ prior treatment. On the next day a total amount of $1.2 \mu \mathrm{g}$ DNA per well was transfected using Lipofectamine2000 (Invitrogen, Karlsbad) according to the manufacturers protocol. For transfection of HEK293 cells the total DNA contained $100 \mathrm{ng}$ of the targeting plasmid UAS TK, which contains Gal4 binding sites and a tyrosine kinase promotor, 2 ng of CMV Renilla, a transfection control under CMV promotor control, the Gal4-tagged protein of interest in different amounts (2 ng -50 ng), and empty pcDNA3.1 vector to reach $1.2 \mu \mathrm{g}$ in total. In the case of HEK293 TK22 cells no UAS TK and 10-200 ng Gal4-tagged protein expressing plasmid were transfected. HEK293 cells were incubated for $24 \mathrm{~h}$ and HEK293 TK22 cells were incubated for $48 \mathrm{~h}$ at $37^{\circ} \mathrm{C} / 5 \% \mathrm{CO} 2$. Then the cells were detached from the wells by pipetting in medium and transferred to $1.5 \mathrm{ml}$ Eppendorf tubes. Cells were pelleted by centrifugation for $5 \mathrm{~min}$ at $3000 \mathrm{rpm}, \mathrm{RT}$. The pellets were resuspended in $100 \mu \mathrm{l}$ of 1x PLB buffer and incubated for $15 \mathrm{~min}$ at RT under constant agitation at $1400 \mathrm{rpm}$. The lysates were centrifuged for $1 \mathrm{~min}$ at $14000 \mathrm{rpm} .20 \mu \mathrm{l}$ of the supernatant was pipetted into one well of a 96-well Optiplate, which was inserted into the PlateChameleon. There $100 \mu \mathrm{l}$ of the "Firefly" solution and afterwards $100 \mu \mathrm{l}$ "Renilla" solution were then added to each well. The luminosity was counted after each step. The measured values were transferred into Microsoft Exel and the Firefly signals were normalized against the Renilla signal and the control transfected cells.

\subsubsection{Subcellular fractionation}

NIH3T3 cells were washed once with ice cold PBS and scraped into $3 \mathrm{ml}$ ice cold PBS per $15 \mathrm{~cm}$ dish. Next the cells were pelleted for $10 \mathrm{~min}$ at $300 \mathrm{xg}, 4^{\circ} \mathrm{C}$ and resuspended in 
$4 \mathrm{ml} / 10^{\wedge} 7$ cells NE buffer A. After 10 min incubation on ice an equal volume NE buffer $\mathrm{B}$ was added, mixed and incubated for another $10 \mathrm{~min}$ on ice. Nuclei were pelleted by centrifugation for $10 \mathrm{~min}$ at $500 \mathrm{xg}, 4^{\circ} \mathrm{C}$. Afterwards the supernatant was saved as cytoplasmic fraction and the nuclear pellet was resuspended in one packed nuclear volume NE buffer $\mathrm{C}$ and was transferred to a dounce homogenisator. The suspension was dounced every 5 min for 20 times until $90 \%$ of nuclei were broken. After centrifugation for $10 \mathrm{~min}$ at $20.000 \mathrm{xg}, 4^{\circ} \mathrm{C}$ the nuclear soluble fraction was transferred to a new tube whereas the pellet was used as nuclear insoluble (chromatin) fraction. Subsequently, the fractions were separated by SDS-PAGE followed by Western Blot analysis.

\subsubsection{Immunoprecipitation}

GeneSwitch NIH3T3 YFP/YFP-ADNP cells were induced with $10 \mathrm{ng} / \mathrm{ml}$ Mifepristone over night. Nuclei of these cells were prepared as in (2.4.2). To solubilize the chromatin fraction, the nuclei were resuspended in $1 \mathrm{ml} / 10^{\wedge} 8$ nuclei PD150 $+10 \mathrm{mM} \mathrm{CaCl}_{2}$ and 10 $\mathrm{U} / \mathrm{ml}$ micrococcal nuclease (Calbiochem) and rotated $37^{\circ} \mathrm{C}$ for $30 \mathrm{~min}$. The reaction was stopped with $1 \mathrm{mM}$ EGTA. The nuclei were broken by sonication for 30min with on/off cycles of $30 \mathrm{~s} / 30 \mathrm{~s}$. The insoluble fraction was pelleted at $10.000 \mathrm{xg}$ for $1 \mathrm{~h}$. The supernatant was split into an input, control (no antibody) and immunoprecipitation (antiGFP) sample. $40 \mu 1$ dynabeads (sheep anti mouse) per sample were $3 \mathrm{x}$ washed in PBS. $5 \mu 1$ mouse anti-GFP antibody were added to the beads and rotated for $3 \mathrm{~h}$ at $4^{\circ} \mathrm{C}$. The beads were then washed $3 \mathrm{x}$ with PD150 buffer and added to the samples. Proteins were immunoprecipitated over night at $4{ }^{\circ} \mathrm{C}$. The antibody/protein complex bound beads were washed 6 times with PD150, separated by SDS-PAGE and analyzed by Western Blot.

\subsubsection{Immunofluorescence}

Cells were seeded on glass coverslips in 6-well plates. For ADNP staining in interphase cells, they were treated with nocodazole ( $200 \mathrm{nM}$, in DMSO) for 5 min prior to fixation. 
Before staining they were washed 2 times with PBS and fixed for 10 min in fixation solution (I) at $37^{\circ} \mathrm{C}$. The coverslips were washed once with PBS and permeabilized for 7 min with permeabilization solution. Cells were washed again once with PBS and blocked for 30 min with blocking solution. Primary and fluorescently labeled secondary antibodies were applied in blocking solution for $1 \mathrm{~h}$ at room temperature / over night at $4^{\circ} \mathrm{C}$ followed by three washing steps with PBS. Thereafter the coverslip was washed three times in water and mounted in mounting medium. Slides were dried overnight at RT and analysed with the confocal microscope Leica TCS SP5.

\subsubsection{Methyltransferase assay}

NIH3T3 cell nuclei were prepared as described in the immunoprecipitation section. The methyltransferase assay was performed according to (Fischle, 2005). Briefly, aliquots of $5 \cdot 10^{6}$ nuclei were centrifuged at $500 \mathrm{xg}, 4{ }^{\circ} \mathrm{C}$, resuspended in $20 \mu \mathrm{l}$ of cold NM buffer and incubated at room temperature for ca. $5 \mathrm{~min} .5 \mu \mathrm{l}$ of 5-S-adenosyl-L$\left[\right.$ methyl $\left.{ }^{3} \mathrm{H}\right]$ methionine in NM buffer were added to start the reaction. After $60 \mathrm{~min}$ incubation at $30{ }^{\circ} \mathrm{C}$ under constant agitation the nuclei were treated with micrococcal nuclease and immunoprecipitated as described in that section (2.4.3). $15 \%$ of the samples were used for Western Blot and $85 \%$ for autoradiography. For autoradiography enhancement EN3HANCE (PerkinElmer) was used as described by the manufacturer. The gel was stained for $10 \mathrm{~min}$ with coomassie solution, incubated $30 \mathrm{~min}$ in destaining solution and another $30 \mathrm{~min}$ in fixation solution (II). The gel was impregnated with scintillators in EN3HANCE for $1 \mathrm{~h}$ and then incubated in precipitation solution for $1 \mathrm{~h}$ at RT. After drying over night in a gel dryer (BIO-RAD) the gel was exposed to an ECL Hyperfilm (GE Healthcare, Buckinghamshire, UK) for 6 months at $-80^{\circ} \mathrm{C}$ and signals were detected using the Kodak X-OMAT 2000. 


\subsection{Biochemical methods}

\subsubsection{SDS-PAGE}

Polyacrylamide gel electrophoresis of proteins in the presence of sodium dodecyl sulfate (SDS-PAGE) was based on the method of Laemmli (Laemmli, 1970). Typically, Trisglycine gels with 10 or $15 \%$ acrylamide-bisacrylamide separating gels were poured and run using the Mini-PROTEAN electrophoresis system (Bio-Rad). Cell pellets, immunoprecipitates and samples from pull-down experiments were resuspended in $1 \mathrm{x}$ Laemmli-buffer and boiled for $5 \mathrm{~min}$. For cell extracts $100 \mathrm{U} / \mathrm{ml}$ Benzonase was included in the 1xLaemmli-buffer and the sample was incubated for $5 \mathrm{~min}$ at room temperature prior to boiling. SeeBlue Plus2 Pre-Stained protein standard (Invitrogen, Karlsbad) was used as size reference. The gel was run in SDS running buffer at $100 \mathrm{~V}$ until the loading dye (bromphenolblue) had left the stacking gel and then at $200 \mathrm{~V}$ until the tracking dye reached the end of the gel.

\subsubsection{Western Blot}

A nitrocellulose membrane, two blot papers and the filter pads were equilibrated in $1 \mathrm{x}$ transfer buffer for $15 \mathrm{~min}$ and the gel was equilibrated in $1 \mathrm{x}$ transfer buffer for $5 \mathrm{~min}$. The sandwich was assembled and fit into a BioRad tank blot chamber which was filled with $1 \mathrm{x}$ transfer buffer. Transfer was run for $1 \mathrm{~h}$ at $100 \mathrm{~V}$ and $4^{\circ} \mathrm{C}$. To check for successful transfer, the membrane was stained for 5 min with Ponceau S Red and washed with water until protein bands became visible. Unspecific protein binding sites of the membrane were blocked in $5 \%$ milk powder in PBS for $1 \mathrm{~h}$ at room temperature. The primary antibody and subsequently the secondary antibody were applied in $2.5 \%$ milk powder in PBST (Table 2-1). Afterwards the membrane was washed three times for $10 \mathrm{~min}$ in PBST. Excess of antibody solution was removed by washing three times in PBST. The membrane was developed by chemiluminiscence using the ECL detection kit (Amersham 
Biosciences). The signals were detected on Amersham ECL Hyperfilms using the Kodak X OMAT.

\subsection{3 generation of oligonucleosomes}

For reconstitution of oligonucleosomes, a biotinylated array of twelve $200 \mathrm{bp}$ long DNA fragments was used, with each subunit containing the 601 nucleosome-positioning sequence (12x200-601). These DNA fragments as well as the scavenger DNA used to prevent oversaturation of the DNA template with histone octamers during assembly (Huynh et al., 2005) were provided by Szabolczs Soeroes (Soeroes, 2010). Histones were expressed according to (Luger et al., 1999) and provided by Winfried Lendeckel.

\section{Introduction of specific histone post-translational modifications}

Specific lysine 9 trimethylation of histone H3 was achieved by native protein ligation according to (Shogren-Knaak and Peterson, 2004). Briefly, $0.2 \mathrm{mM}$ of truncated H3 protein (amino acids 21 to 135$)$ with a point mutation of alanine 21 to cysteine $(\mathrm{H} 3 \Delta 1-20$ $\mathrm{A} 21 \mathrm{C}$ ) and $1 \mathrm{mM}$ of an $\mathrm{N}$-terminal $\mathrm{H} 3$ peptide fragment with the desired $\mathrm{H} 3 \mathrm{~K} 9 \mathrm{me} 3$ mark and a C-terminal thioster group were ligated in peptide ligation buffer for $24 \mathrm{~h}$ at $25^{\circ} \mathrm{C}$ with vigorous mixing. The reaction mix was diluted 50-fold into SAU-200 buffer, applied to a $5 \mathrm{ml}$ Hi-Trap SP-Sepharose high performance cation exchange column (GE Healthcare), and eluted with a linear $\mathrm{NaCl}$ gradient from 200 to $600 \mathrm{mM}$ in 10 column volumes. Fractions, containing the ligated product were identified by SDS-PAGE and Coomassie staining and verified by ESI mass spectrometry. The protein was dialyzed extensively against $2 \mathrm{mM}$ DTT at $4^{\circ} \mathrm{C}$, lyophilized and stored at $-80^{\circ} \mathrm{C}$.

\section{Reconstitution of histone octamers}


Core histone octamers were reconstituted as described in (Luger et al., 1999). Briefly, lyophilized purified wt core histones $\mathrm{H} 2 \mathrm{~A}, \mathrm{H} 2 \mathrm{~B}, \mathrm{H} 4$ and wt or modified $\mathrm{H} 3$ were dissolved in unfolding buffer and mixed to equimolar ratios. The histone mix was dialyzed at $4^{\circ} \mathrm{C}$ against $\mathrm{RB}$ high buffer with at least three changes of dialysis buffer. Histone octamers were concentrated to $10-20 \mathrm{mg} / \mathrm{ml}$ using an Amicon Ultra centrifugal filter unit (Millipore) and purified over a HiLoad 16/60 Superdex 200 prep grade gelfiltration column (GE Healthcare) on a ÄKTA Purifier or ÄKTA Explorer FPLC instrument (GE Healthcare) from H2A-H2B dimers. Peak fractions with pure histone octamers were pooled, concentrated to at least $2 \mathrm{mg} / \mathrm{ml}\left(\mathrm{OD}_{276}=0.9\right)$ and stored in $50 \%$ $\mathrm{v} / \mathrm{v}$ glycerol at $-20^{\circ} \mathrm{C}$.

\section{Reconstitution of recombinant oligonucleosomes}

Reconstitution of oligonucleosomes was carried out according to (Huynh et al., 2005; Luger et al., 1999) at $4^{\circ} \mathrm{C}$. Firstly, glycerol was removed from the octamers by dialyzing for at least $3 \mathrm{~h}$ against RB high buffer. For reconstitution of oligonucleosomes, 12x200601 DNA templates (in the presence of equimolar scavenger DNA) were mixed with histone octamers in a molar ratio of 1.0 to 1.1 octamers per DNA in $500 \mu 1 \mathrm{RB}$ high buffer. Reaction mixture was first dialyzed against RB high buffer using a dialysis tube with a molecular weight cutoff of 3500. Nucleosome assembly was performed by continuously replacing RB high buffer against RB low buffer for $72 \mathrm{~h}$ using a peristaltic pump as described in (Luger et al., 1999). Reconstituted oligonucleosomes were extensively dialyzed against TEAE buffer and stored at $4^{\circ} \mathrm{C}$.

\subsubsection{Recombinant proteins}

ADNP-FLAG was expressed using the TNT® Quick Coupled Transcription/Translation System (Promega, Madison, USA). The TNT® Quick Master Mix was thawed on ice. $20 \mu \mathrm{l}$ master mix, $0.5 \mu \mathrm{l}$ methionine, $0.5 \mu \mathrm{g}$ pADNP-FIFl-HAHA - DNA and $\mathrm{H}_{2} \mathrm{O}$ to $25 \mu 1$ were mixed per precipitation and incubated for $90 \mathrm{~min}$ at $30^{\circ} \mathrm{C}$. 
Recombinant HP1 variants were obtained from Szabolczs Soeroes (Soeroes, 2010).

\subsubsection{Peptide pull-down}

To detect binding of proteins to modified histone peptides, $1 \mu \mathrm{g}$ of the biotinylated peptides was bound to $40 \mu \mathrm{l}$ streptavidin coated beads for $3 \mathrm{~h}$ at RT, $1400 \mathrm{rpm}$. To remove excess peptides the beads were washed $3 \mathrm{x}$ with PBS. $0.5 \mathrm{ml}$ of nuclear extract (prepared as in 2.4.3) or $20 \mu \mathrm{l}$ of a in vitro transcription and translation reaction (TNT Quick coupled Transcription/Translation System) +/- recombinant HP1 in $0.5 \mathrm{ml}$ PD150 were incubated with the peptide-bound beads overnight at $4^{\circ} \mathrm{C}$ under constant rotation. On the next day the beads were washed $6 \mathrm{x}$ with $1 \mathrm{ml} \mathrm{PD} 150$ at $4^{\circ} \mathrm{C}$. Proteins, which were bound to the beads were analyzed by SDS-PAGE followed by Western Blot analysis.

\begin{tabular}{lll}
\hline Name & Protein (aa) & Sequence \\
\hline H3unmod & Histone H3 (1-20) & MARTKQTARKSTGGKAPRKQ \\
H3K9me3 & Histone H3 (1-20) & MARTKQTARKme3STGGKAPRKQ \\
ADNPunmod & ADNP (759-778) & EDDSYEARKSFLTKYFNKQP \\
ADNPK767me3 & ADNP (759-778) & EDDSYEARKme3SFLTKYFNKQP \\
\hline
\end{tabular}

Table 2-4: Peptides used for the pulldown experiment

\subsection{6 chromatin pull-down}

SILAC HeLa S3 nuclear extracts (unlabeled, light or labeled with Arg-13C15N(+10) and Lys-13C(+6), heavy) were prepared as described (Ong and Mann, 2006) and provided by the Group of Prof. Dr. Reinhard Lührmann at the Max Planck Institute for Biophysical Chemistry.

$50 \mu \mathrm{g}$ of biotinylated nucleosomal arrays were pre-incubated with $200 \mu \mathrm{l}$ of streptavidin coated magnetic beads (Promega) in CPD buffer for $4 \mathrm{~h}$ at $4^{\circ} \mathrm{C}$. Unbound 
oligonucleosomes were removed by three washes for $5 \mathrm{~min}$ with CPD buffer. Immobilized nucleosomal arrays were incubated with $5 \mathrm{ml}$ SILAC HeLa S3 nuclear extracts (first experiment: unmodified nucleosomal arrays with light extract and H3K5me3 arrays with heavy extract; second experiment vice versa) for $4 \mathrm{hr}$ at $4^{\circ} \mathrm{C}$. Beads were then washed 6 times with CPD buffer and bound proteins were eluted by boiling the beads in Laemmli buffer. The heavy and light samples were mixed, run on SDS-PAGE gels, stained with Coomassie and analyzed by mass spectrometry.

For Western Blot analysis $10 \mu \mathrm{g}$ of biotinylated oligonucleosomes were bound to $40 \mu 1$ of streptavidin coated magnetic beads (Promega) and incubated with nuclear extract (prepared as for 2.4.3) as described above. CPD buffer was replaced by PD150.

\subsubsection{Mass spectrometry and analysis}

The mass spectrometry analysis was performed by the mass spectrometry facility of Dr. Henning Urlaub at the Max Planck Institute for Biophysical Chemistry.

Entire gel lanes of the SDS PAGE gels from 2.5.6 were cut into 23 slices of equal size. Proteins within the slices were digested according to (Shevchenko et al., 1996). Peptides were extracted and analyzed by LC-coupled tandem MS on an Orbitrap X1 mass spectrometer (Thermo Fisher Scientific). Data analysis was performed as described (Sharma et al., 2009). Briefly, raw data files analyzed with the MaxQuant software (version 1.0.12.31), which performs peak list generation, SILAC-based quantitation, false discovery rate (FDR) determination, peptide to protein group assembly and data filtration. Data were searched against a concatenated forward and reversed version of the human International Protein Index (IPI) database (version 3.52) Mascot (Matrix Science). The accepted FDR was $1 \%$ for the proteins and peptides. For peptides shared among different identified proteins, SILAC ratios were only considered for the ratio of the protein identified with the highest number of unique peptides. Output files were subtracted according to the gi-numbers (NCBI) of the found proteins with the help of the 
statistical program R. An arbitrary cut-off of at least 2 fold enrichment in both experiments per protein was used.

$\mathrm{R}$ program used for data analysis:

open " $\mathrm{R}$ " (D1=experiment1, D2=experiment 2 with reversed ratio)

D1=read.table("/Users/kmosch/Desktop/exp1.txt", sep="\t", header=T)

D2=read.table("/Users/kmosch/Desktop/exp2.txt", sep="\t", header=T)

D1_D2=merge(D1,D2, by.x=1, by.y=1) \#\#\#\#\#\# combining tables by GI number 


\section{Results}

\subsection{Identification of factors associated with H3K9me3}

To gain insight into mechanisms for heterochromatin formation and maintenance I tried to identify proteins involved in heterochromatin formation by quantitative mass spectrometry analysis of a chromatin pulldown experiment. In vitro reconstituted chromatin consisting of 12 nucleosomes assembled on an artificial nucleosome positioning sequence (Lowary and Widom, 1998; Thastrom et al., 1999), trimethylated or unmodified at lysine 9 of histone $\mathrm{H} 3$ was immobilized on magnetic beads. These beads were incubated with stable isotope $\left(\mathrm{Arg}-{ }^{13} \mathrm{C}^{15} \mathrm{~N}\right.$ and $\mathrm{Lys}{ }^{13} \mathrm{C}=$ heavy) labeled and normal (= light) HeLa S3 nuclear extracts respectively. The bound proteins, separated by SDSPAGE were digested with trypsin and subjected to MS/MS analysis. The stable isotope labeling of amino acids in cell-culture (SILAC) (Ong and Mann, 2005, 2006) allowed for quantitative determination of factors enriched at the H3K9me3 oligonucleosomes. To exclude factors that are enriched in either the heavy or the light extract or which do not bind reproducibly to the modified oligonucleosomes, a reverse experiment using light HeLa S3 nuclear extract for unmodified H3 and light extract for the oligonucleosomes trimethylated at lysine 9 was conducted. The overlap of proteins with a greater than 2fold enrichment in the $\mathrm{H} 3 \mathrm{~K} 9$ trimethylated sample was determined and further analyzed (Figure 3-1). 


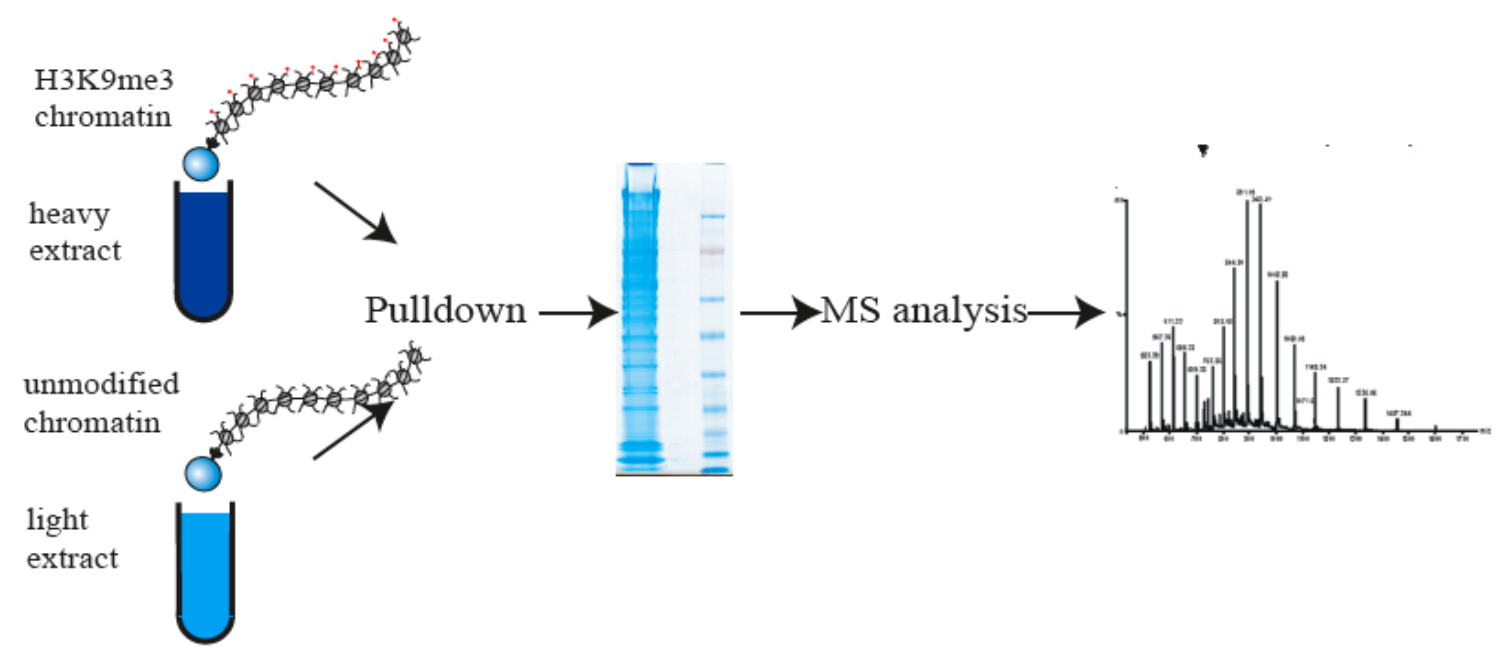

Figure 3-1: experimental setup

Arg $-{ }^{13} \mathrm{C}^{15} \mathrm{~N}$ and Lys ${ }^{13} \mathrm{C}$ labeled (heavy) and unlabeled (light) nuclear extracts were used for pulldown experiments with nucleosomal arrays biotinylated at one end and immobilized on streptavidin coated beads. Red dots represent lysine 9 trimethylation on H3. The two samples were mixed and separated by SDSPAGE. Proteins in gel slices were proteolyticaly fragmented and analyzed by LC-MS/MS.

In the initial experiment 490 proteins were identified but only for 355 of them a heavy to light $(\mathrm{H} / \mathrm{L})$ ratio could be determined. 208 of those factors displayed less than 2 -fold enrichment in either the modified or unmodified sample. 106 proteins with more than 2fold enrichment were detected in that pulldown experiment (Figure 3-2 A). In the reverse experiment 535 proteins were identified, 405 of those with an calculatable $\mathrm{H} / \mathrm{L}$ ratio. 248 proteins were not enriched more than 2-fold at modified or unmodified chromatin whereas 79 proteins were enriched in the H3K9me3 fraction (Figure 3-2 B).

The overlap of both experiments consists of 20 proteins including known H3K9me3 interaction partners such as all isoforms of HP1 (CBX1, 3 and 5) and ICBP90 (UHRF 1 and 2) (Table 3-1). 
CHAPTER 3 - Results
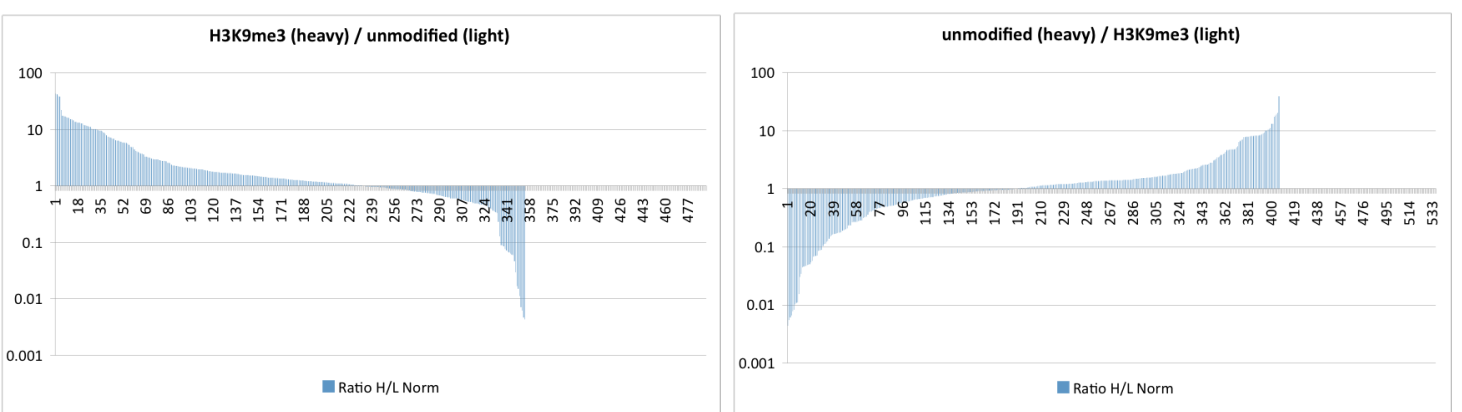

Figure 3-2: Distribution of the ratios between the heavy and light samples

The ratios between the heavy and light samples were calculated for each protein, ordered by value and plotted beginning with the proteins enriched at $\mathrm{H} 3 \mathrm{~K} 9 \mathrm{me} 3$.

\begin{tabular}{|l|l|l|}
\hline name & $\begin{array}{l}\text { ratio } \\
\text { H3K9me3 heavy } \\
\text { unmodified light }\end{array}$ & $\begin{array}{l}\text { ratio } \\
\text { H3K9me3 light } \\
\text { unmodified heavy }\end{array}$ \\
\hline CBX5 & 14 & 5 \\
\hline UHRF1 & 12 & 4 \\
\hline UHRF2 & 12 & 5 \\
\hline MED31 & 10 & 4 \\
\hline UBP7 & 8 & 6 \\
\hline CBX1 & 8 & 6 \\
\hline DNMT1 & 7 & 6 \\
\hline CBX3 & 7 & 7 \\
\hline ADNP & 4 & 12 \\
\hline CAF1B & 4 & 13 \\
\hline POGZ & 4 & 6 \\
\hline ZN580 & 4 & 3 \\
\hline JHD1A & 3 & 3 \\
\hline CA103 & 3 & 7 \\
\hline DYL1 & 3 & 3 \\
\hline CIR1A & 2 & 4 \\
\hline WDR75 & 2 & 4 \\
\hline NOL11 & 2 & 2 \\
\hline WDR43 & 2 & 3 \\
\hline UTP15 & 2 & 4 \\
\hline
\end{tabular}

Table 3-1: Proteins with at least 2-fold encichment in both experiments 
One factor that which had so far not been identified as associated with heterochromatin was the activity dependent neuroprotector ADNP. Therefore this protein was selected for further studies.

\subsection{Verification of $\mathrm{H} 3 \mathrm{~K} 9 \mathrm{me} 3$ association of $A D N P$}

\subsubsection{ADNP associates with H3K9me3 and pericentromeric heterochromatin}

In order to verify the enrichment of ADNP at oligonucleosomes trimethylated at H3K9, I repeated the chromatin pulldown experiment described above. Western Blot analysis with an ADNP specific antibody showed specific binding to H3K9me3 but not to unmodified oligonucleosomes (Figure 3-3 A). The detection of one ADNP band instead of two was not reproducible and might be due to low pulldown efficiency. Detection of histone H4 served as a loading control for the unmodified and methylated oligonucleosomes. To test if this specificity depends only on the trimethylation of $\mathrm{H} 3 \mathrm{~K} 9$ or on the DNA/nucleosome context, I performed a peptide pulldown. In this experiment peptides containing the first 21 amino acids of histone H3 were used instead of oligonucleosomes. Again specific binding of ADNP to $\mathrm{H} 3 \mathrm{~K} 9 \mathrm{me} 3$ in contrast to the unmodified peptides was observed (Figure 3-3 B). 
$\mathbf{A}$

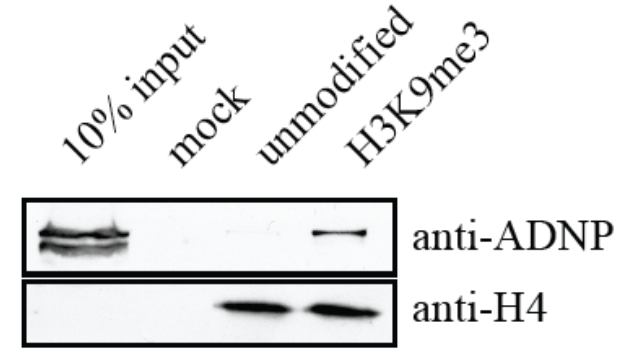

B

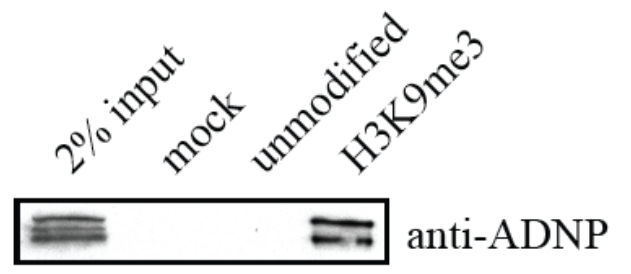

Figure 3-3: ADNP associates with H3K9me3

A, Chromatin pulldown. Recombinant 12-mer oligonucleosomal arrays reconstituted with the indicated H3 species were immobilized on magnetic beads and incubated with HeLa S3 nuclear extract. Beads without coupled oligonucleosomes were used as control (mock). Western Blots with the indicated antibodies are shown. B, Peptide pulldown. Histone H3 peptides (aa 1-21) unmodified or trimethylated at lysine 9 were bound to magnetic beads and incubated with HeLa S3 nuclear extract. Beads without immobilized peptides served as a control (mock). Western Blot with anti-ADNP antibody is shown.

In vivo the localization of ADNP was analyzed by immunostaining using an anti-ADNP antibody. DNA was counterstained with DAPI to visualize heterochromatic areas, which are mainly pericentromeric heterochromatin. The ADNP staining in interphase cells revealed a speckled distribution in the cell nucleus with enrichment at DAPI-dense regions. In the cytoplasm, a tubulin-like staining was observed (Figure 3-4 A). During mitosis and until telophase ADNP is largely displaced from chromatin. The tubulin-like staining however, does not allow conclusions about whether ADNP is completely excluded from chromatin or remains at microtubuli attachment sites (Figure 3-5). Furthermore, the distribution of ADNP in fibroblasts was investigated by subcellular fractionation. The cytoplasm and the nuclear soluble (nucleoplasm) and insoluble (chromatin) fractions were probed with an anti-ADNP antibody. $\beta$-tubulin was used as cytoplasmic marker and the transcription factor TBP and histone $\mathrm{H} 3$ as nuclear/chromatin markers. ADNP was enriched in the nuclear/chromatin fraction. However, no ADNP could be detected in the cytoplasmic fraction with $\beta$-tubulin (Figure 3-4 B). 
A

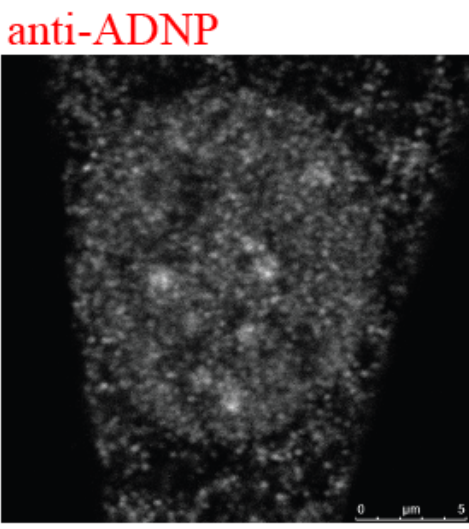

DAPI

merge
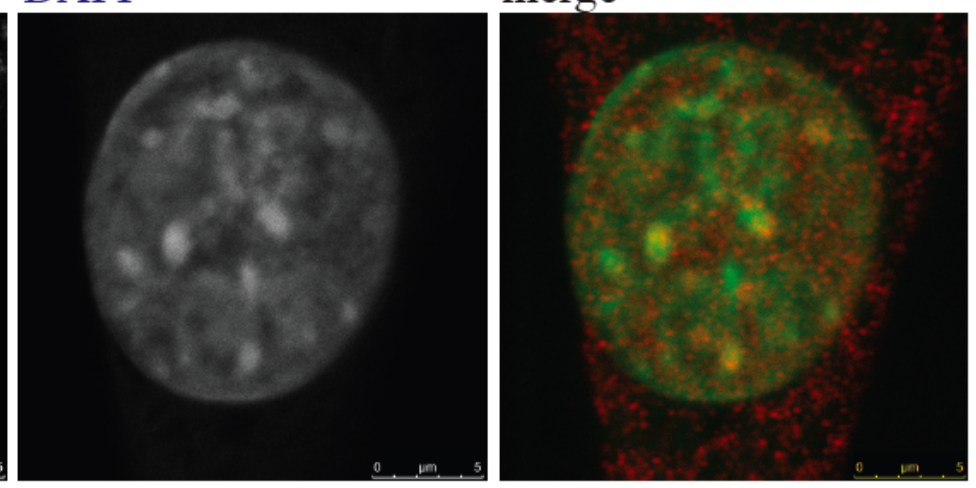

\section{B}

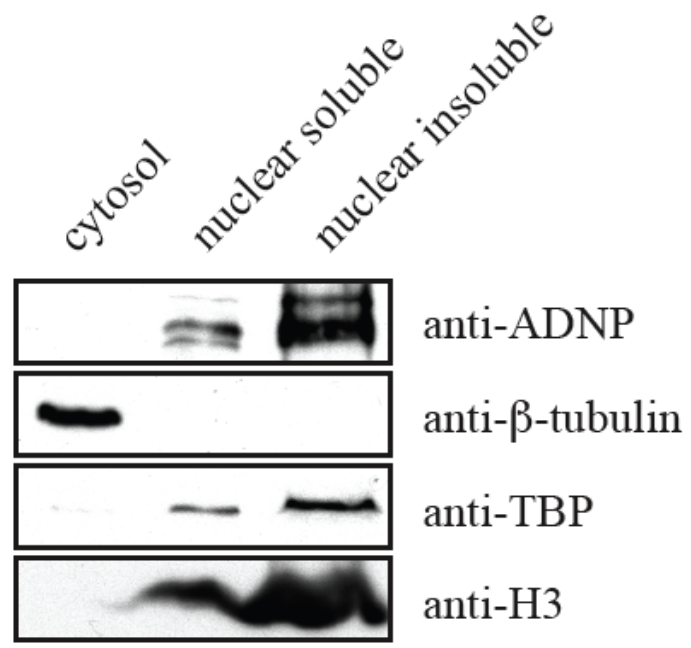

Figure 3-4 ADNP is enriched at pericentromeric heterochromatin

A, Immunofluorescence. NIH3T3 cells were stained with anti-ADNP antibody and DNA was visualized using DAPI. Bars represent $5 \mu \mathrm{m}$. B, Subcellular fractionation. The cytoplasmic fraction of NIH3T3 cells was extracted with $0.1 \%$ Triton X-100. Nuclei were lysed under salt-free conditions and the soluble and insoluble fractions were separated by centrifugation. Western Blots with the indicated antibodies are shown. 


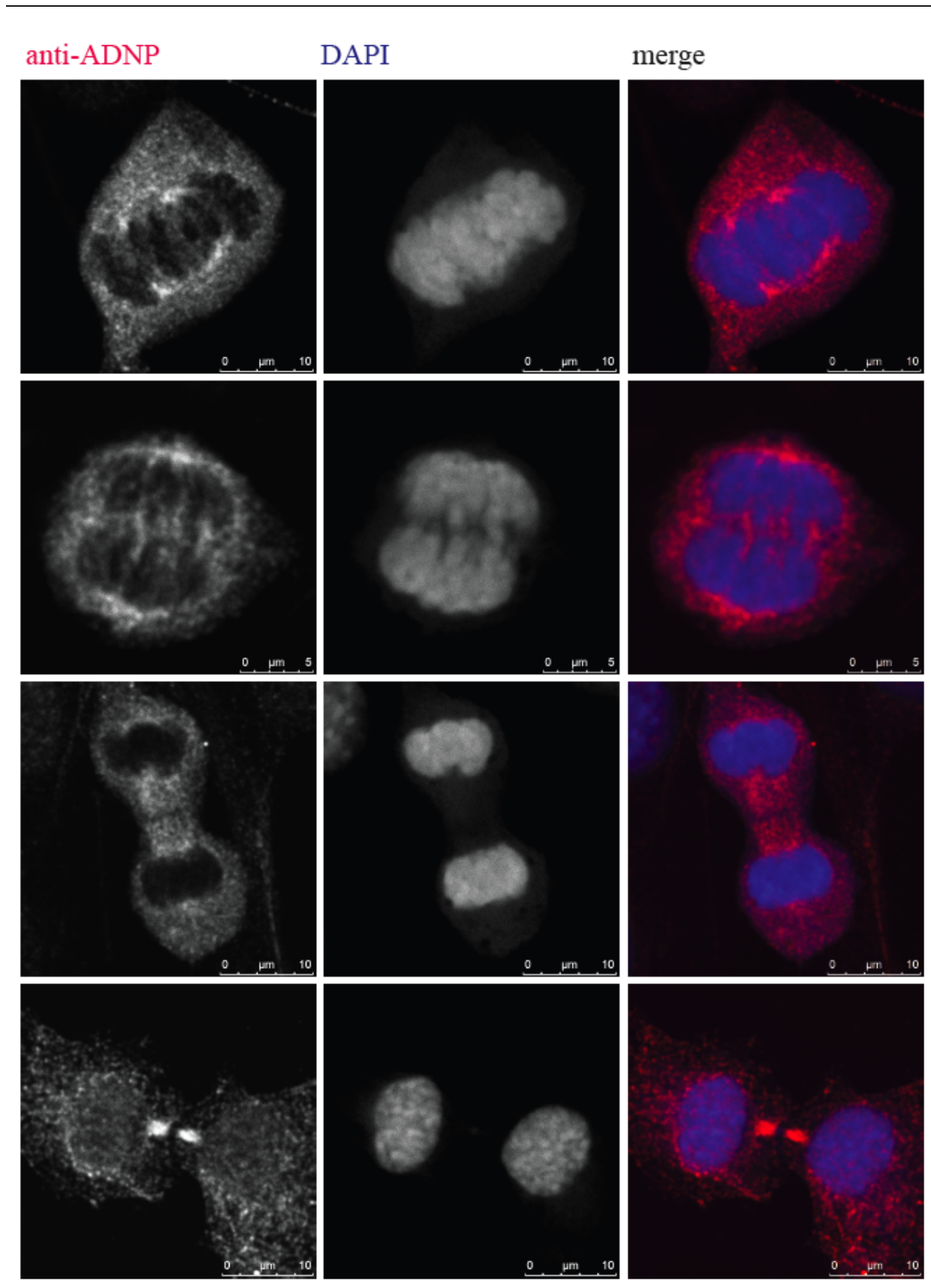

Figure 3-5: Localization of ADNP during mitosis

Immunofluorescence. NIH3T3 cells were stained with an anti-ADNP antibody. DNA was visualized using DAPI. Bars represent $10 \mu \mathrm{m}$ (rows 1, 3, 4) and $5 \mu \mathrm{m}$ (row 2). 
Returning to the initial observation that ADNP associates with $\mathrm{H} 3 \mathrm{~K} 9 \mathrm{me} 3$, I investigated their co-localization in cells. Co-staining with anti-ADNP and anti-H3K9me3 antibodies in mouse embryonic fibroblast (MEF) cells showed co-localization at DAPI-dense regions. Since trimethylation of H3K9 cannot be removed from the cells directly, I repeated the staining in Suv39h1/h2 double knockout cells. Suv39 is the methyltransferase that is responsible for $\mathrm{H} 3 \mathrm{~K} 9 \mathrm{me} 2 / 3$ at pericentromeric heterochromatin (Peters et al., 2001). Consequently, no enrichment of H3K9me3 at DAPI-dense areas could be seen in those cells. Interestingly, ADNP was found removed from pericentromeric heterochromatin whereas the speckled distribution in the nucleus remained (Figure 3-6).

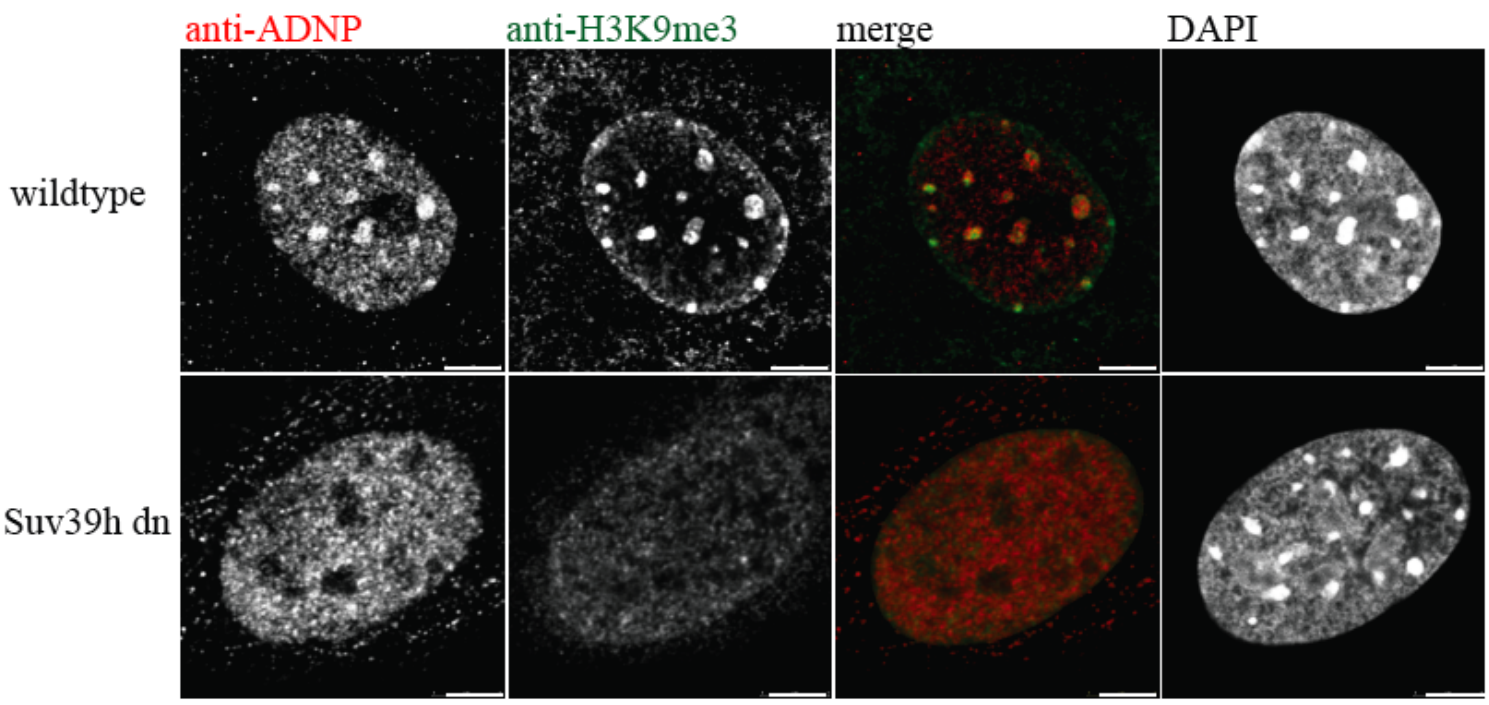

Figure 3-6: ADNP localization to pericentromeric heterochromatin depends on Suv39h

Immunofluorescence. MEF wildtype and Suv39h1/h2 double knockout cells were stained with the indicated antibodies. DNA was visualized using DAPI. Bars represent $5 \mu \mathrm{m}$.

In conclusion, $\mathrm{ADNP}$ associates with $\mathrm{H} 3 \mathrm{~K} 9 \mathrm{me} 3$ oligonucleosomes and histone peptides in vitro. The protein is localized to the cell nucleus, where it co-localizes with $\mathrm{H} 3 \mathrm{~K} 9 \mathrm{me} 3$ at pericentromeric heterochromatin. This localization depends on the H3K9 methyltransferase Suv39. 


\subsubsection{ADNP is not alternatively spliced in mouse fibroblasts}

Western Blots with anti-ADNP antibodies showed a double band after a $15 \%$ SDSPAGE (Figure 3-3) which could be further resolved by 10\% SDS-PAGE (Figure 3-7 B). Therefore, I tested whether alternative splicing occurs in mouse fibroblasts. Figure 3-7 A shows a schematic representation of the genomic structure of ADNP. An untranscribed exon is followed by two small (108 bp and $93 \mathrm{bp}$ ) and one major exon. To see if one of the two smaller exons is removed by alternative splicing, I designed primers surrounding each of these exons. mRNA was extracted from MEF and NIH3T3 cells, reverse transcribed and analyzed by PCR. Primer-pair 1 surrounding exon 2 amplifies a 199 bp fragment from the full-length transcript and a $91 \mathrm{bp}$ fragment if exon 2 is lacking. Primerpair 2 amplifies a 198 bp or a 105 bp fragment from a transcript with or without exon 3 respectively. In both cases only the PCR product corresponding to the full-length transcript could be detected (Figure 3-7 C). 
A

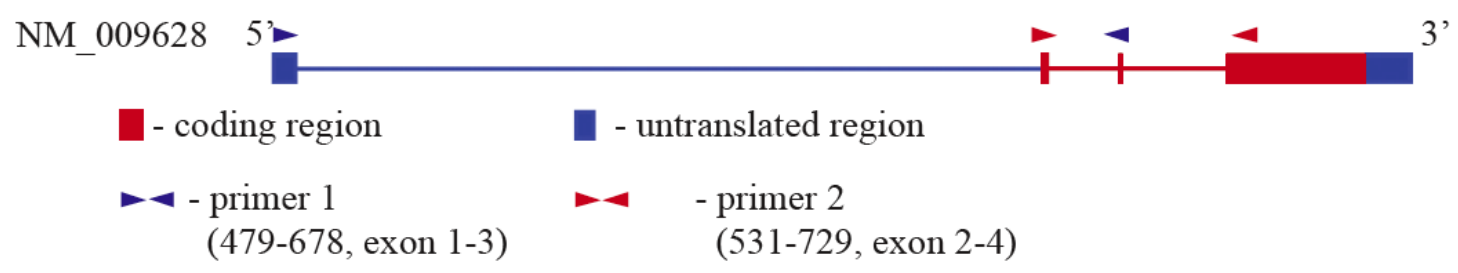

B

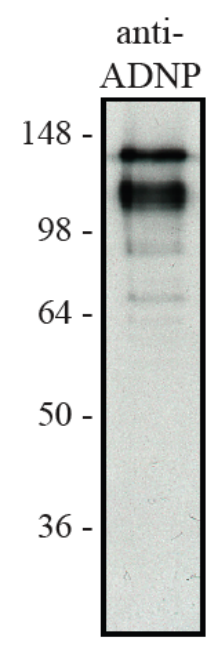

C

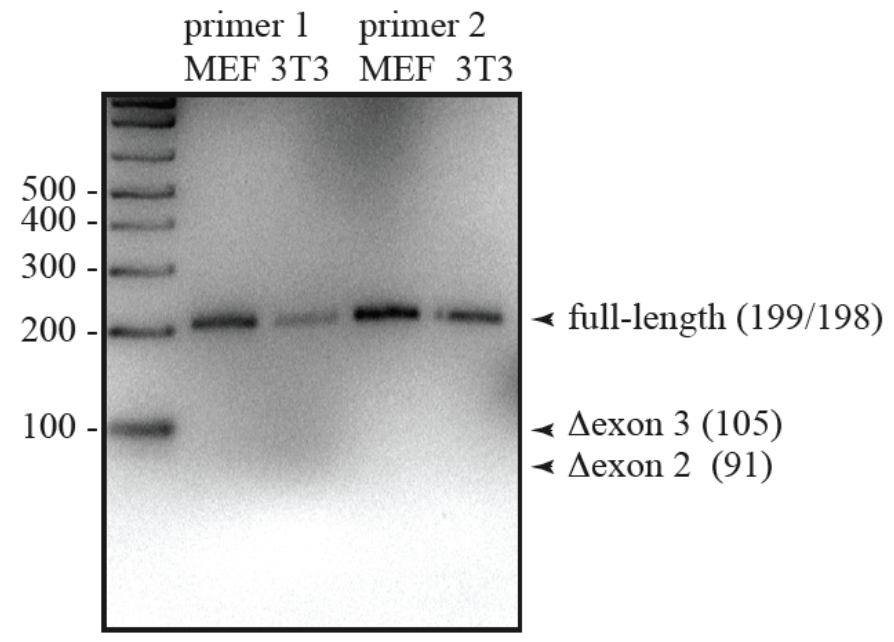

Figure 3-7: Only full-length ADNP mRNA is detected in mouse fibroblasts.

A, Schematic representation of the genomic structure of ADNP. Exons are drawn as boxes while introns are shown as lines. Red and blue arrowheads indicate the location of the primers used in C. B, Western Blot. Whole cell extract was separated by $15 \%$ SDS-PAGE and analyzed by Western Blotting using an anti-ADNP antibody (BD Biosciences). C, RT-PCR. cDNA from NIH3T3 (3T3) cells and mouse embryonic fibroblasts (MEF) was used as template for the PCR reaction with the primers shown in A. The PCR products separated on a 1\% agarose-gel stained with ethiduim bromide are shown. Expected sizes of the full-length cDNA and the alternatively spliced variants are indicated.

From this experiment, I concluded that there are no alternatively spliced ADNP transcripts in mouse fibroblasts. 


\subsection{Mode of ADNP recruitment to heterochromatin}

ADNP does not contain a chromo domain or another known domain that is known to bind histone modifications. However, ADNP was reported to interact with the SWI/SNF complex via its C-terminal domain (Mandel and Gozes, 2007). Brg1, one component of that complex was recently shown to have functions in pericentromeric heterochromatin (Bourgo et al., 2009). Co-precipitation of ADNP with HP1 $\alpha$ has also been reported (Mandel et al., 2007). The ADNP homeodomain contains a PxVxL motif, a known HP1 interaction motif. It also includes an ARKS sequence, similar to that of the histone $\mathrm{H} 3$ tail, which can be trimethylated at lysine 9 and then be bound by HP1. Therefore the homeodomain is likely to be an HP1 interaction domain, allowing indirect targeting of ADNP to pericentromeric heterochromatin by HP1.

To determine how ADNP is recruited to pericentromeric heterochromatin I used deletion mutants, which identified the homeodomain as necessary and sufficient for localization. Peptide pull-down experiments with ADNP expressed in reticulocyte lysate showed that ADNP does not bind to $\mathrm{H} 3 \mathrm{H} 9$ me3 directly but only in the presence of HP1. The ADNPHP1 interaction could be mapped to the PxVxL but not the ARKS motif.

\subsubsection{ADNP localization to chromocenters depends on the Homeodomain}

To identify the domain that is responsible for ADNP localization, I cloned different deletion constructs. Shown in Figure 3-8 are CFP-ADNP constructs containing fulllength ADNP, the protein lacking the homeodomain, and the homeodomain alone, all containing the nuclear localization signal (NLS). These constructs were co-expressed with RFP-HP1 $\beta$ as a heterochromatin marker in NIH3T3 cells. Full-length ADNP and the homeodomain alone co-localized with HP1 whereas the mutant lacking the homeodomain did not display an enrichment at any particular site in the nucleus. 
A

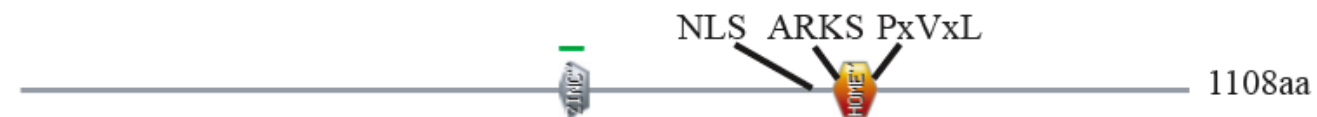

B

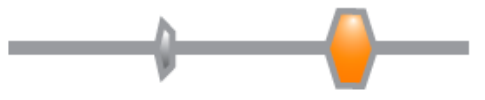

\section{CFP-ADNP}

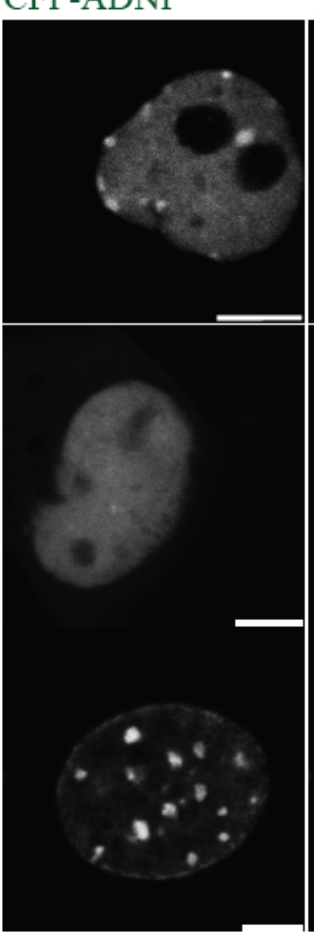

RFP-HP1 $\beta$

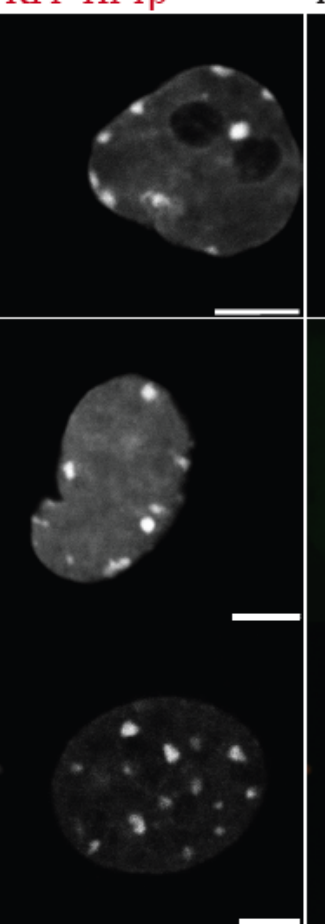

merge
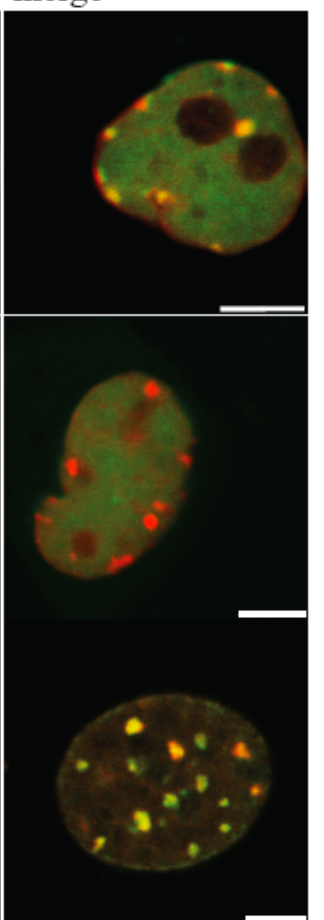

Figure 3-8: The homeodomain of ADNP is necessary and sufficient for localization to pericentromeric heterochromatin

A, Schematic representation of the ADNP protein structure as predicted by PROSITE (www.expasy.ch/cgibin/prosite). Positions of the zinc-finger (grey), the homeodomain (orange), the nuclear localization signal (NLS) and the ARKS and PxVxL motifs are indicated. B, Live cell imaging. NIH3T3 cells were transfected with CFP-ADNP, CFP-ADNP $\triangle 741-846$ (homeodomain deletion), CFP-ADNP-701-846 (NLS and the homeodomain) and RFP-HP1 $\beta$. Bars represent $5 \mu \mathrm{m}$. 


\subsubsection{ADNP does not bind to H3K9me3 directly but is targeted to pericentromeric heterochromatin by HP1}

Since the homeodomain contains the putative HP1 interaction motifs, I asked whether ADNP is able to bind to H3K9me3 directly or only in the presence of HP1. I performed a peptide pull-down experiment using $\mathrm{H} 3 \mathrm{~K} 9 \mathrm{me} 3$ and unmodified $\mathrm{H} 3$ peptides immobilized to magnetic beads, and beads without peptides as a control. FLAG-tagged ADNP was expressed in TNT reticulocyte extract and incubated with these beads in the absence and presence of recombinant HP1. In the absence of HP1, ADNP did not bind to the beads or peptides. When co-incubated with $\mathrm{HP} 1 \alpha, \mathrm{HP} 1 \beta$ and HP1 $\gamma$, ADNP bound specifically to the methylated peptide bound beads together with each of the HP1 isoforms (Figure 3-9).

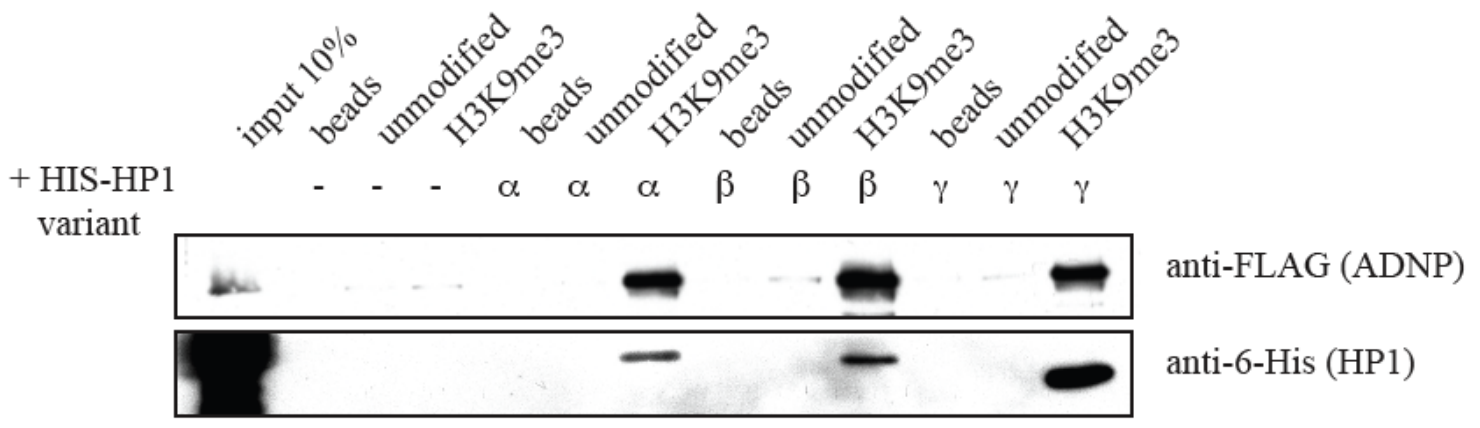

Figure 3-9 All three HP1 isoforms are able to recruit ADNP to H3K9me3

Peptide pulldown. Flag-tagged ADNP was expressed in TNT-reticulocyte extract and incubated with histone H3 peptides (unmodified or trimethylated at lysine 9), immobilized on magnetic beads. $1 \mu \mathrm{g}$ recombinant, HIS-tagged HP1 was added as indicated. Beads without peptide served as a control. The pulldown was analyzed by Western Blotting using anti-FLAG and anti-6-HIS antibodies. 
To test whether the isoform independent recruitment of ADNP by HP1 in vitro is also reflected in vivo, I used HP1 knockout cells. Antibody staining of ADNP and visualization of DNA with DAPI in HP1 $\alpha$, HP1 $\beta$ and HP1 $\gamma$ single knockout MEF cells showed that ADNP localization is not affected by the lack of one HP1 isoform (Figure 3-10 A). In HP1 $\alpha / \beta$ double knockout MEF cells HP1 $\gamma$ is the only isoform expressed, and HP1 $\gamma$ did not localize to chromocenters in these HP1 $\alpha / \beta$ knockout cells. Also in these cells, no enrichment of ADNP at pericentromeric heterochromatin could be detected (Figure 3-10 B). 
A

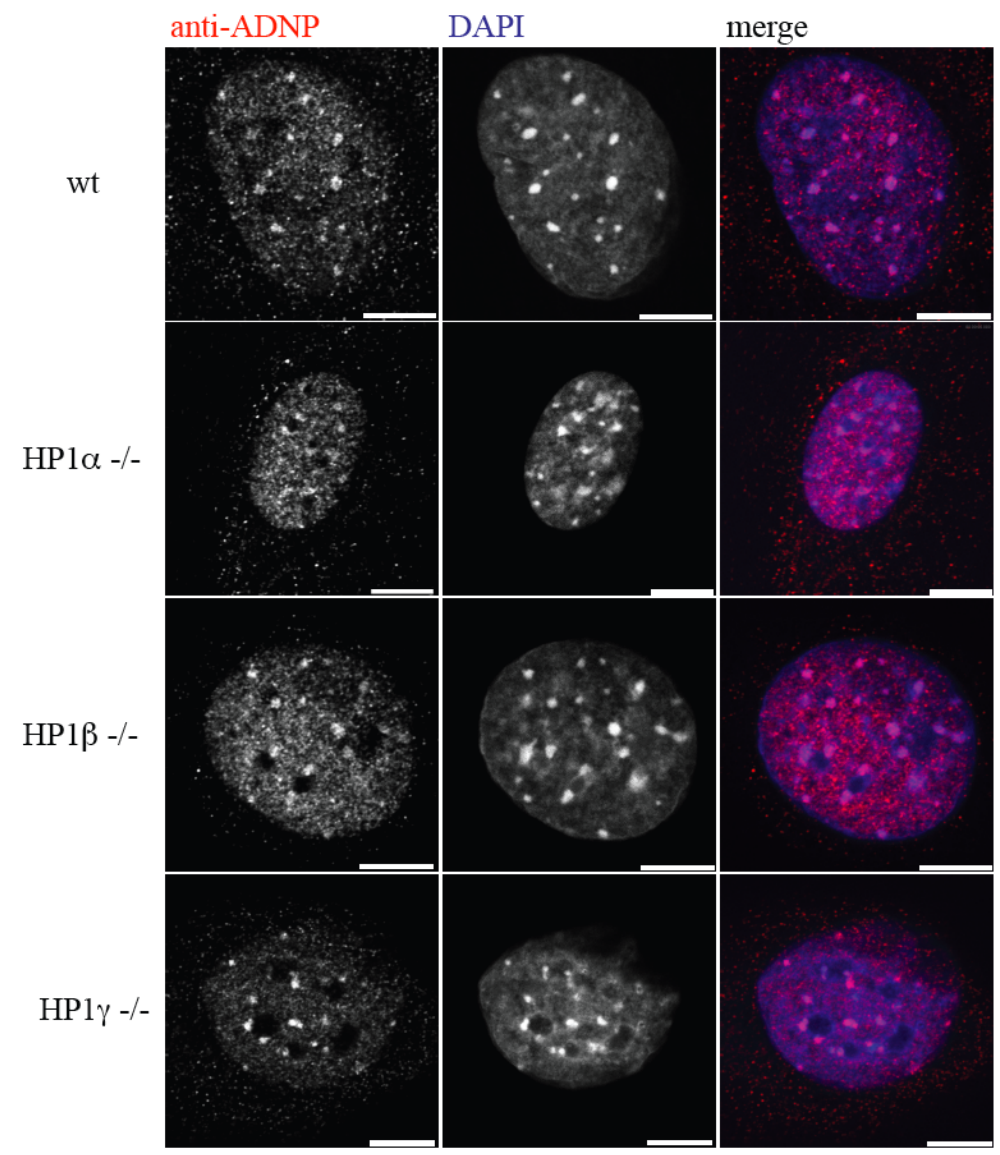

B

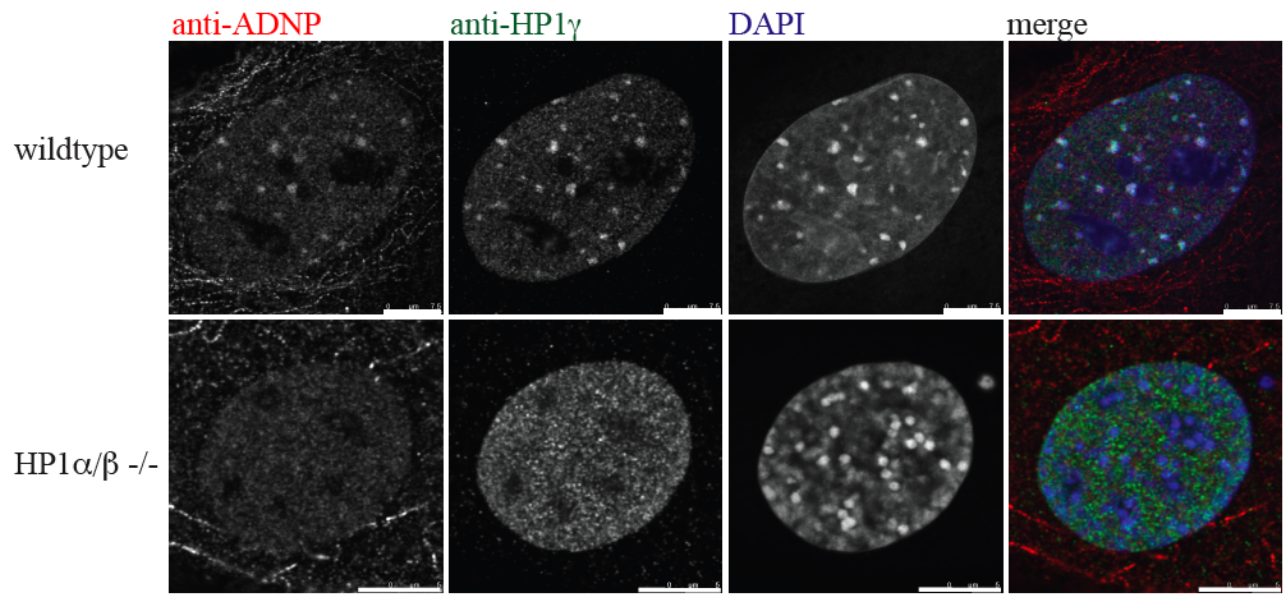

Figure 3-10: ADNP localization to pericentromeric heterochromatin is HP1 dependent.

A, Immunofluorescence. MEF wildtype and $\mathrm{HP} 1 \alpha, \beta$ or $\gamma$ single knock-out cells were stained with an antiADNP antibody. B, Immunofluorescence. MEF wildtype and HP1 $\alpha / \beta$ double knock-out cells were stained with the indicated antibodies. DNA was visualized using DAPI. Bars represent $7.5 \mu \mathrm{m}$. 
To verify that ADNP displacement in HP1 $\alpha / \beta$ double knockout cells is not an effect of H3K9me3, ADNP or HP1 $\gamma$ misregulation their expression levels were assessed by Western Blot. Total cell extracts of wild type and HP1 $\alpha / \beta$ knockout MEF cells were separated by SDS-PAGE and probed with anti-ADNP, anti-HP1 $\gamma$ and anti-H3K9me3 antibodies. $\beta$-tubulin and $\mathrm{H} 4$ were used as a loading control. No significant changes in the expression / modification levels could be detected. Immunostaining of H3K9me3 and counterstaining with DAPI in the HP1 $\alpha / \beta$ double knockout cells did not indicate an effect on H3K9me3 localization (Figure 3-11). 
$\mathbf{A}$
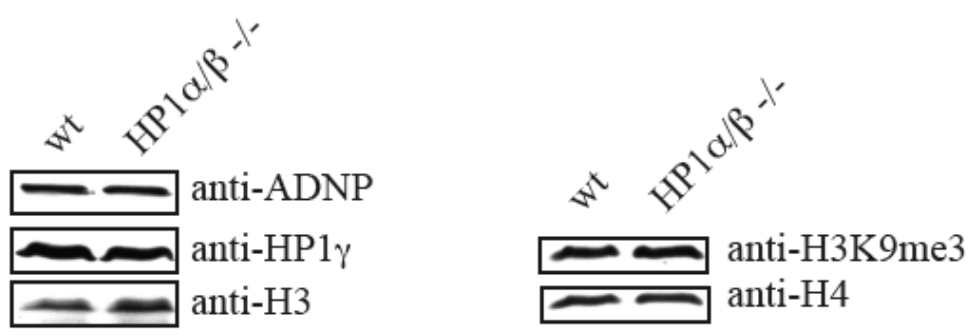

B

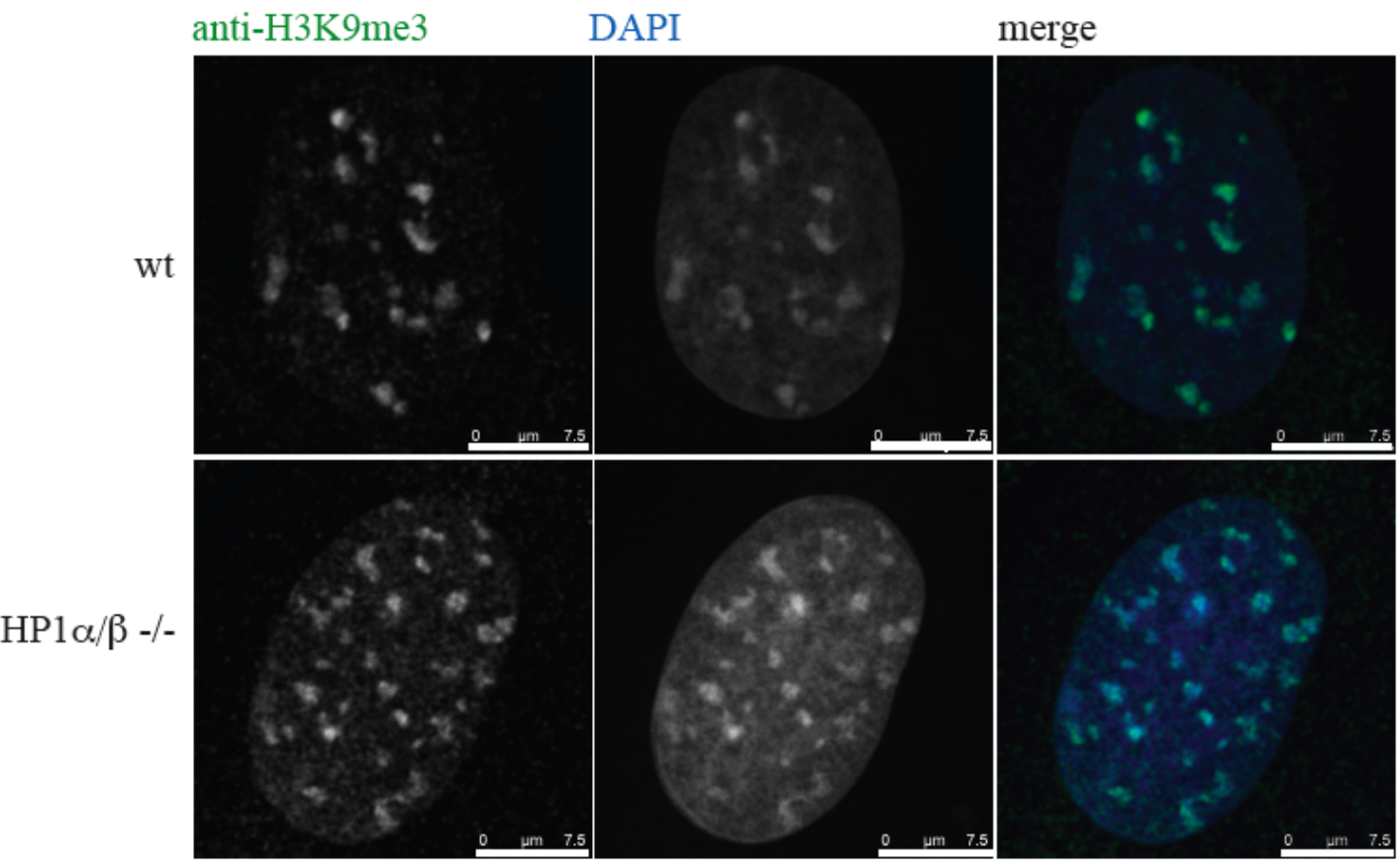

Figure 3-11: levels of HP1 $\gamma$, ADNP and H3K9me3 as well as H3K9me3 distribution are not affected by HP1 $\alpha / \beta$ knockout

A, Western Blot. Total cell extracts of wild type and HP1 $\alpha / \beta$ knockout MEF cells were analyzed by Western Blot using the indicated antibodies. B, Immunofluorescence. MEF wildtype and HP1 $\alpha / \beta$ double knockout cells were stained with anti-H3K9me3 antibodies. DNA was visualized using DAPI. Bars represent $7.5 \mu \mathrm{m}$.

From these results, I concluded that ADNP does not bind to H3K9me3 directly but is recruited to pericentromeric heterochromatin by HP1. This behavior is not dependent on one particular isoform of HP1. 


\subsubsection{ADNP localization to chromocenters mainly depends on HP1 binding to the PxVxL motif within the homeodomain}

To further characterize the ADNP - HP1 interaction, the homeodomain was mutated at the putative HP1 interaction sites. The lysine in the ARKS motif was mutated to arginine to prevent a possible methylation event, which might be bound by the HP1 chromo domain. In the PxVxL motif the valine was mutated to a glutamate. This mutation has been shown to strongly reduce binding of the HP1 chromoshadow domain (Figure 3-12 A). Wild type ADNP, the single point mutants and the double mutant were fused to YFP and introduced into NIH3T3 cells. Because the transfection efficiency of the full-length protein was below $1 \%$ and strong overexpression caused aggregation of YFP-ADNP (data not shown) inducible stable cell-lines were established. Figure 3-12 B shows Western Blot analysis of two cell-lines for each of the mutations using an anti-ADNP antibody. The upper band represents the YFP-ADNP fusion protein whereas the lower band shows the endogenous ADNP. For comparison, extracts of untransfected NIH3T3 cells were loaded. Only moderate overexpression levels were detected (Figure 3-12 B). Live cell imaging revealed that the ARKS point mutation had no effect on ADNP localization. The mutation within the PxVxL motif lead to a strong reduction of the ADNP signal at chromocenters. Only the K767K/V821E double mutant was completely displaced. These results were confirmed in vitro. Wild type and mutant FLAG-tagged ADNP was expressed in TNT reticulocyte extract and used for $\mathrm{H} 3 \mathrm{~K} 9 \mathrm{me} 3$ peptide pulldown experiments. In the presence of recombinant HP1 $\beta$ wild type and K767R mutated ADNP were bound to H3K9me3 peptide bound beads. However, only minimal amounts of the PxVxL mutant proteins and no double mutant protein could be detected (Figure 3-12 D). 
A

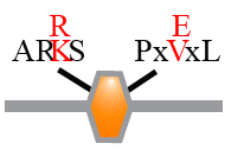

B

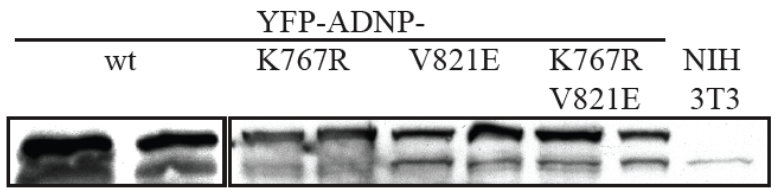

anti-ADNP

C

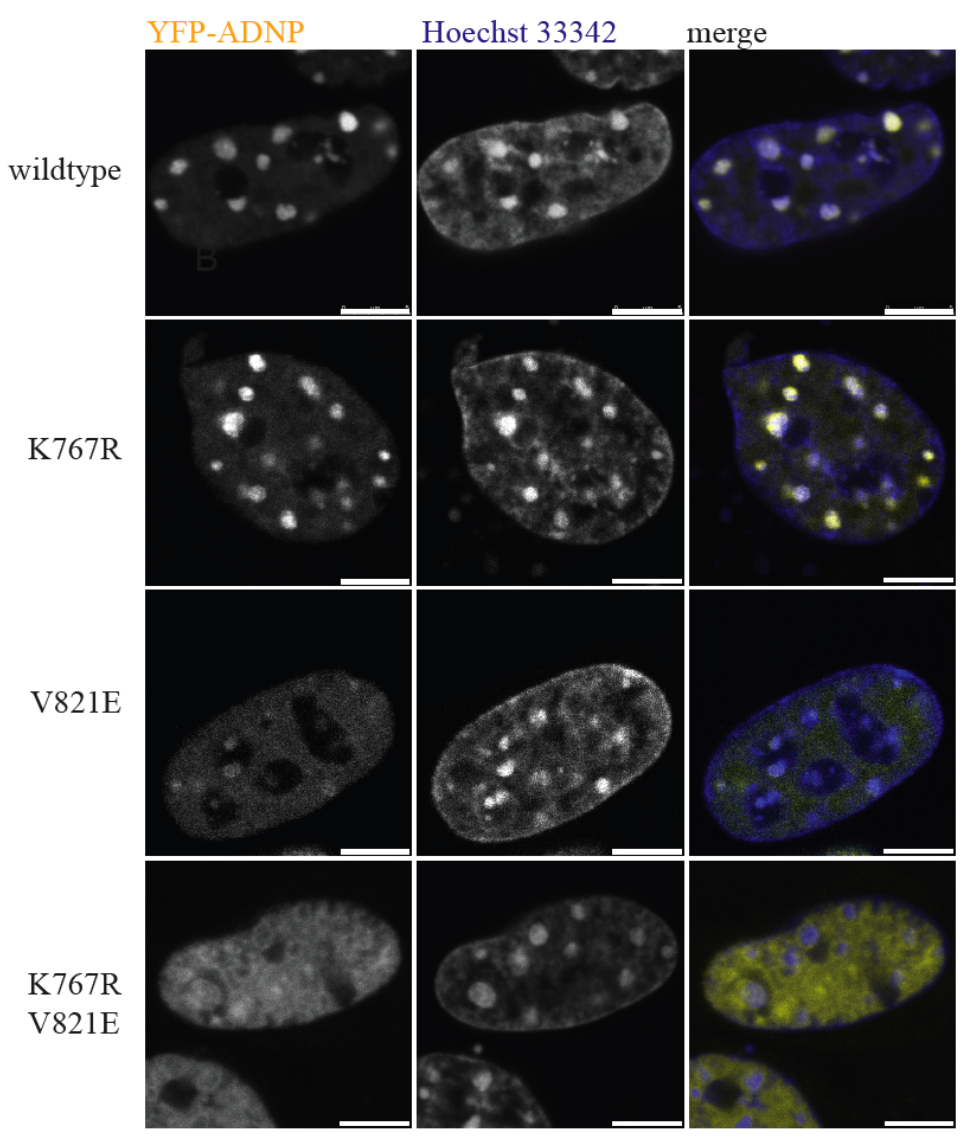

D

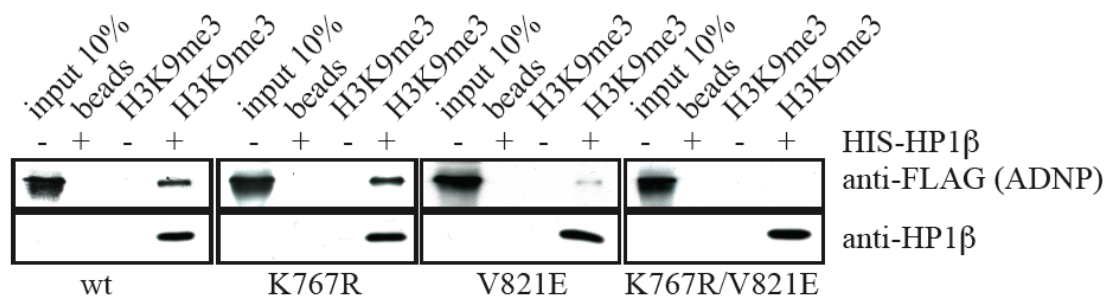

Figure 3-12: The PxVxL and ARKS motifs are involved in ADNP localization.

A, Schematic representation of the ADNP-homeodomain, containing a $\mathrm{K}$ to $\mathrm{R}$ mutation in the ARKS and a $\mathrm{V}$ to E mutation in the PxVxL motif. B, Two clones of inducible NIH3T3 cells, expressing YFP-ADNP, wild-type or carrying one or both of these mutations were analyzed by Western Blot using an anti-ADNP antibody. C, Live cell imaging. DNA was stained with Hoechst 33342 dye. Bars represent $5 \mu$ m. D, Pulldown experiment with H3K9me3-peptides, immobilized on magnetic beads using TNT-reticulocyte-extract expressed ADNP-FLAG (wt and point mutants) and recombinant 6xHIS-HP1 $\beta$, analyzed by Western Blot. 
To test whether ADNP dimerization/multimerization rather then HP1 interaction is involved in recruitment of the ADNP mutants, YFP-ADNP was immunoprecipitated using an anti-GFP antibody. All forms of YFP-ADNP including the double mutant, which does not localize to pericentromeric heterochromatin, co-immunoprecipitated endogenous ADNP.

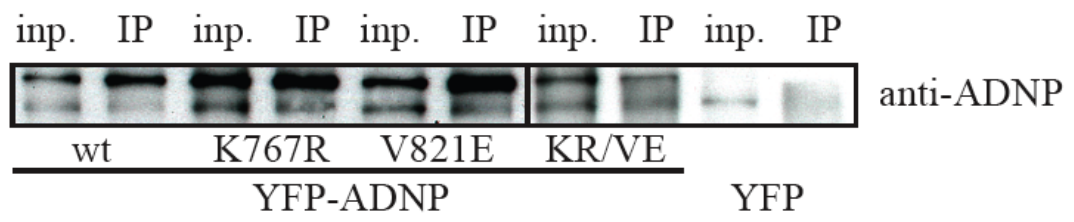

Figure 3-13: The AKRS and PxVxL point mutations have no influence on ADNP multimerization.

Immunoprecipitation. Nuclear extract was prepared from inducible NIH3T3 cells, expressing YFP-ADNP, wild-type or carrying a $\mathrm{K}$ to $\mathrm{R}$ mutation in the ARKS (K767R) and a V to E mutation in the PxVxL (V821E) motif. YFP expressing inducible NIH3T3 cells were used as control. Immunoprecipitation was performed with anti-GFP antibody and analyzed by Western Blot using anti-ADNP antibody.

If the interaction between the HP1 chromoshadow domain and the ADNP PxVxL motif is the main mechanism for its recruitment to $\mathrm{H} 3 \mathrm{~K} 9 \mathrm{me} 3$, then association of ADNP with H3K9me3 should be strongly reduced by a mutation within the chromoshadow domain. A mutation of tryptophan 170 to alanine (W170A) of HPI $\beta$ had been described to abolish HP1-PxVxL interaction without affecting HP1 dimerization and H3K9me3 binding (Thiru et al., 2004). This mutant as well as wild type HP1 $\beta$ were used in a peptide pulldown experiment with wild type FLAG-tagged ADNP. The W170A mutation had no effect on HP1 binding to H3K9me3 peptide bound beads. However, only background levels of ADNP were detected with HP1 $\beta$ (W170A). 


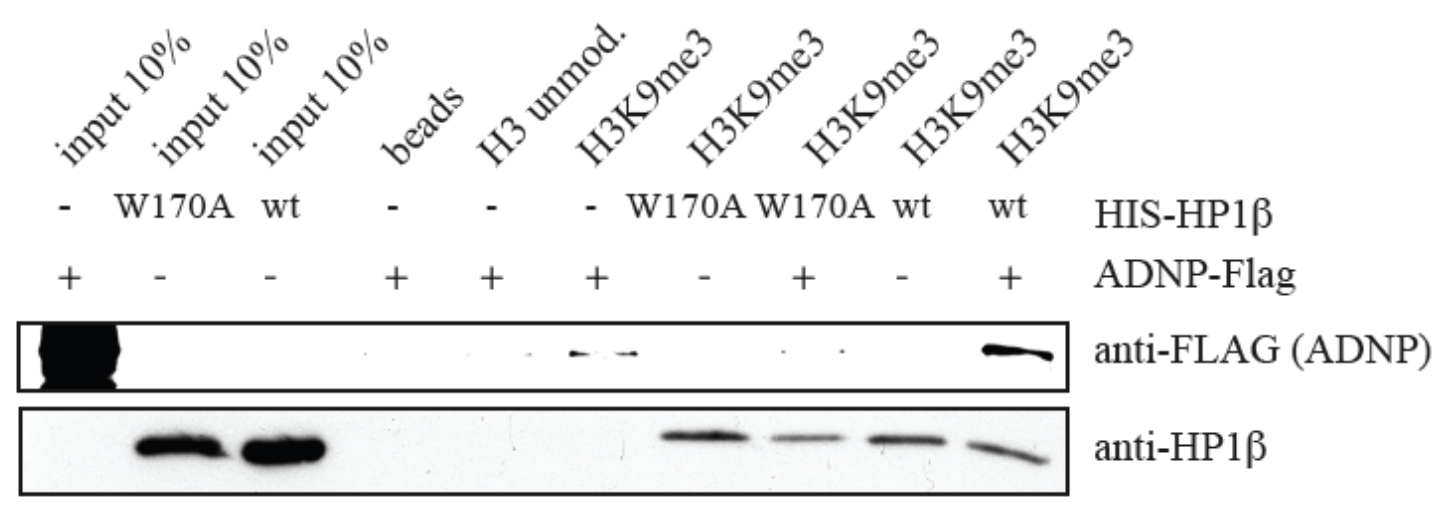

Figure 3-14: HP1 chromoshadow domain mutation strongly reduces binding of ADNP

Pulldown experiment with H3K9me3-peptides, immobilized on magnetic beads using TNT-reticulocyteextract expressed ADNP-FLAG and recombinant 6xHIS-HP1 $\beta$ (wt and W170A mutants), analyzed by Western Blot. 


\subsubsection{A possible lysine methylation in the ADNP ARKS motif is not involved in HP1 binding}

The results of the previous section showed a severe effect of PxVxL mutation on ADNP recruitment. This effect was enhanced by a mutation within the ARKS motif (Figure 3-12). Thus, a minor effect of a possible lysine methylation of the ADNP ARKS motif could not be excluded so far. Therefore, I tested whether ADNP is methylated in nuclei and HP1 would bind trimethylated lysine 767 .

To test if ADNP is methylated, nuclei were isolated from NIH3T3 cells and incubated with adenosyl-L-methionine S-[methyl- $\left.{ }^{3} \mathrm{H}\right] . \quad$ Subsequently ADNP was immunoprecipitated with an anti-ADNP antibody. $15 \%$ of the sample was used to confirm the immunoprecipitation by Western Blot. The remaining sample was used for SDS-PAGE followed by autoradiography. After 6 months detection time a signal at the expected size of ADNP was detected in the IP. Compared to the signal of nonspecifically precipitated histones in the IP, and the control the possible ADNP signal was very weak.

The possibility of HP1 binding to trimethylated lysine 767 of ADNP was assessed by pull-down experiments using unmethylated ADNP peptides or peptides trimethylated at lysine 767. Histone $\mathrm{H} 3$ peptides unmodified or trimethylated at lysine 9 served as positive controls. Using HeLaS3 nuclear extracts in the peptide pull-down experiment all isoforms of HP1 bound to H3K9me3 but not detectably to ADNPK767me3 peptide bound beads. The same result was observed for ADNP itself and for CDYL (Fischle et al., 2008), another chromodomain protein. 
A

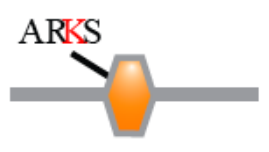

B

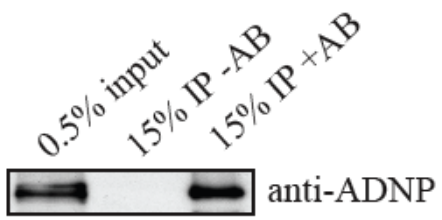

C

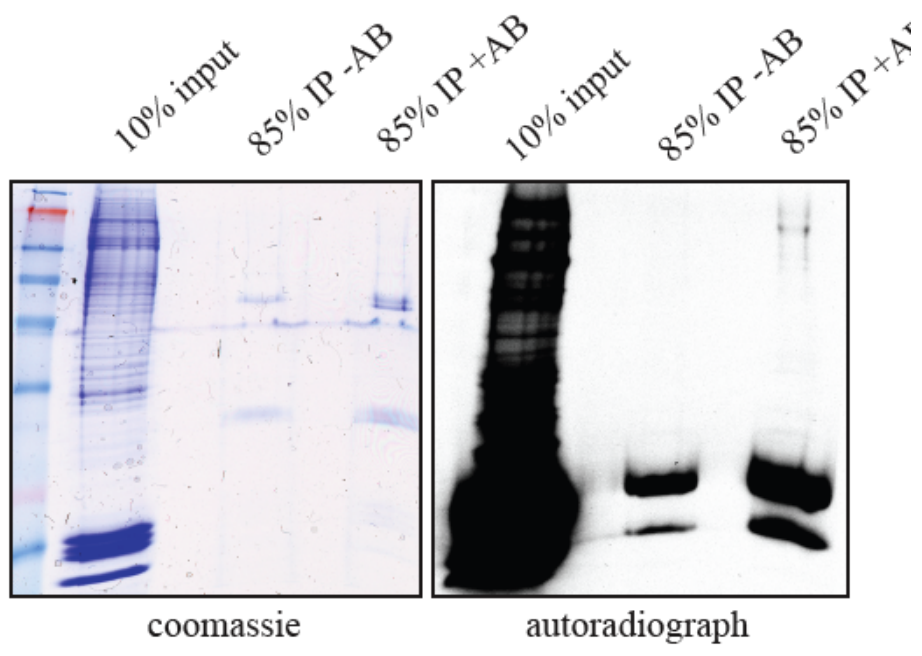

D

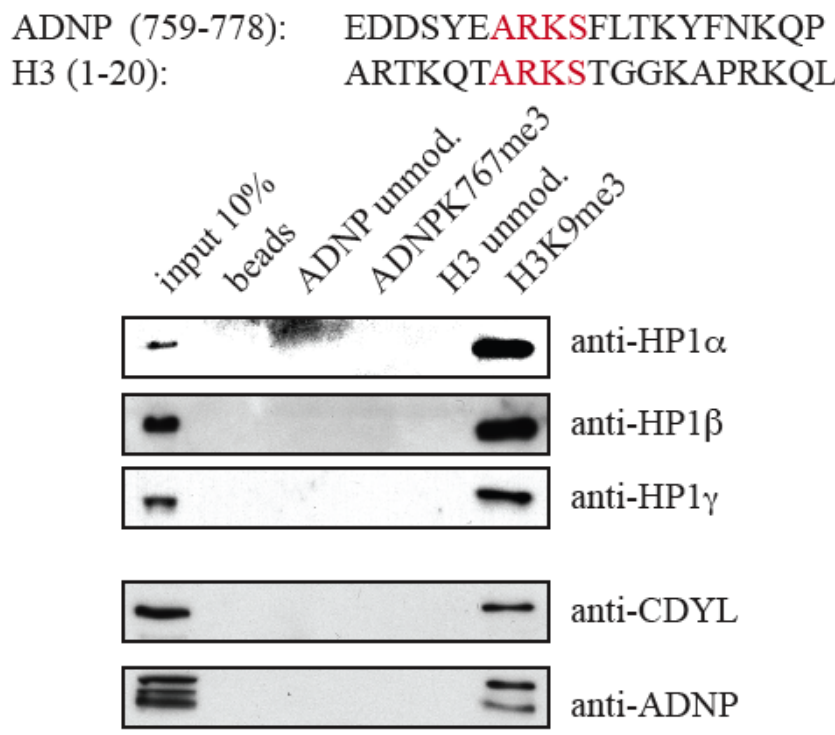

Figure 3-15: The ARKS motif of ADNP is not involved in HP1 binding

A, Schematic representation of the ADNP homeodomain containing the ARKS motif. B and C, Immunoprecipitation. Isolated nuclei of NIH3T3 cells were incubated with adenosyl-L-methionin S[methyl- $\left.{ }^{3} \mathrm{H}\right]$. Immunoprecititation was performed with anti-ADNP antibody and analyzed by $\mathbf{B}$, Western Blot with anti-ADNP antibody and $\mathbf{C}$, autoradiography. Coomassie staining and the autoradiograph after 6 months exposure are shown. D, Peptide pulldown. ADNP peptides and histone H3 peptides unmodified or trimethylated at lysine 767 or 9 respectively, were bound to magnetic beads and incubated with HeLa S3 nuclear extract. Beads without immobilized peptides served as a control. A Western Blot with the indicated antibodies anti-ADNP antibody is shown. 
Taken together, these results indicate that ADNP is recruited to pericentromeric heterochromatin by HP1 interacting with $\mathrm{H} 3 \mathrm{~K} 9 \mathrm{me} 3$ via its chromo domain on one hand and with the homeodomain of ADNP on the other hand. The HP1 - ADNP interaction is mainly mediated by binding of the HP1 chromoshadow domain to the PxVxL motif within the ADNP homeodomain. Although the effect of a PxVxL mutation is enhanced by an additional mutation in the ARKS motif, there is no evidence that methylation of the ARKS motif is involved in that process.

\subsection{ADNP function at pericentromeric heterochromatin}

ADNP had been shown to be involved in up- and down-regulation of a diverse set of genes during embryogenesis (Mandel et al., 2007). Other chromatin functions have not been described so far. Known interaction partners of ADNP however lead to testable hypotheses of ADNP function at pericentromeric heterochromatin. Brg1, an interaction partner of ADNP, has recently been shown to be involved in structure and organization of pericentromeric heterochromatin (Bourgo et al., 2009). In addition, ADNP is displaced from chromocenters in Suv39h1/h2 knockout cells. An ADNP loss-of-function phenotype at pericentromeric heterochromatin should also be reflected in those cells. Therefore, I tested whether ADNP knockdown results in one of the phenotypes described for the Suv39h1/h2 knockout cells: (I) change of global levels and distribution of histone modifications, (II) reduction of DNA methylation and (III) transcriptional silencing of major satellite repeats (Lehnertz et al., 2003; Peters et al., 2003; Peters et al., 2001; Schotta et al., 2004). 


\subsubsection{ADNP knockdown does not phenocopy the Brg1 knockout phenotype}

Deletion of $\mathrm{Brg} 1$ in mouse fibroblasts results in dissolution of pericentromeric heterochromatin domains and a redistribution of trimethylated $\mathrm{H} 3 \mathrm{~K} 9$ and H4K20 (Bourgo et al., 2009). Involvement of ADNP in these Brg1 functions was tested by knockdown of ADNP by siRNA. Two different siRNAs, 71 and 68, were used to knock down ADNP. A scrambled siRNA served as control. The knockdown of ADNP was confirmed by immunofluorescence staining using an anti-ADNP antibody. DAPI staining of DNA did not show dissolution of pericentromeric heterochromatin (Figure 3-16, Figure 3-17). The distribution of $\mathrm{H} 3 \mathrm{~K} 9 \mathrm{me} 3$ and $\mathrm{H} 4 \mathrm{~K} 20 \mathrm{me} 3$ remained unchanged in the ADNP knockdown cells as well (Figure 3-16, Figure 3-17). 


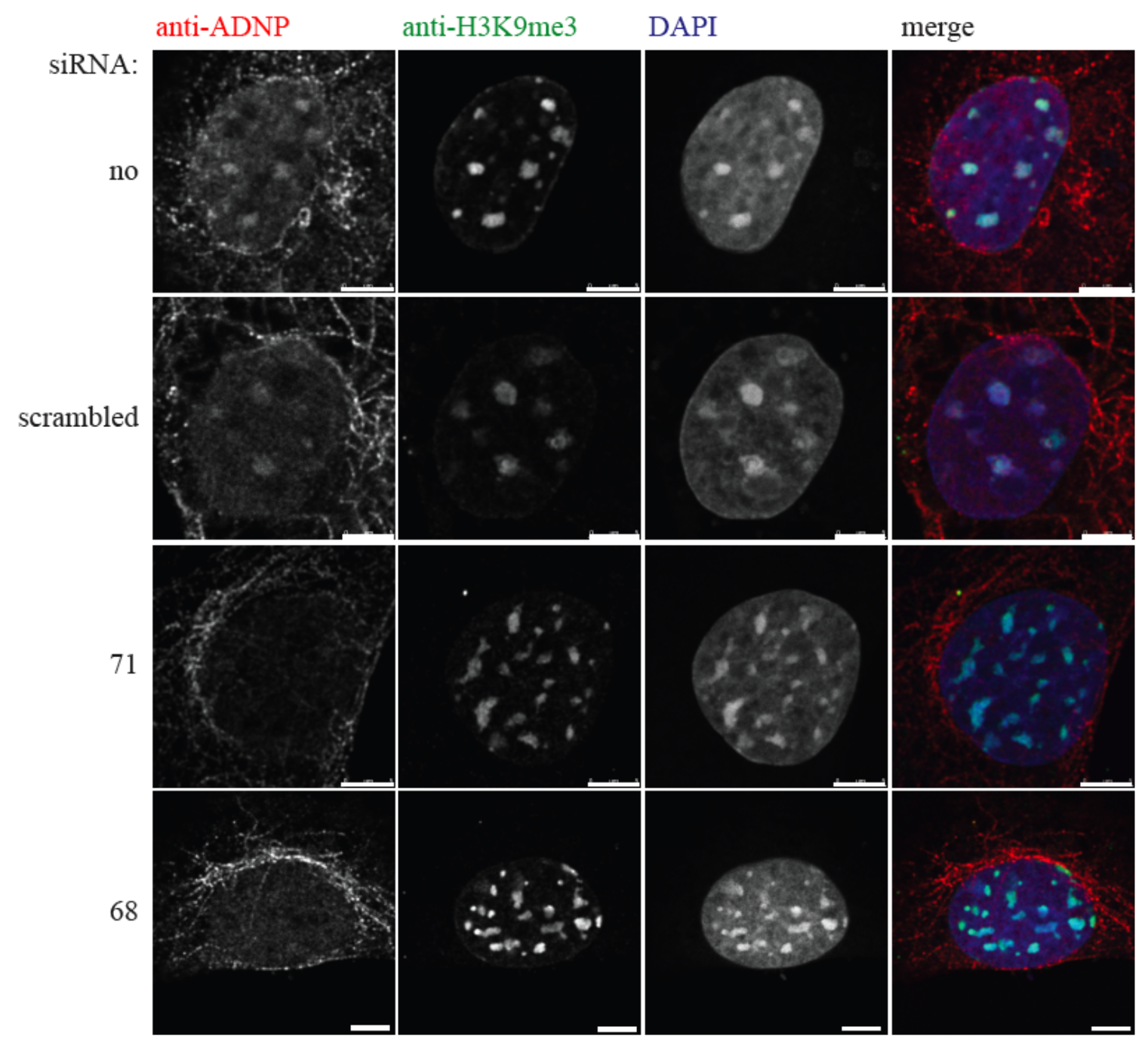

Figure 3-16: ADNP knockdown does not influence H3K9me3 localization.

Immunofluorescence. NIH3T3, transfected with siRNA against ADNP (68 and 71), scrambled siRNA or untransfected (no) were stained with the indicated antibodies. DNA was visualized with DAPI. Bars represent $5 \mu \mathrm{m}$. 
CHAPTER 3 - Results

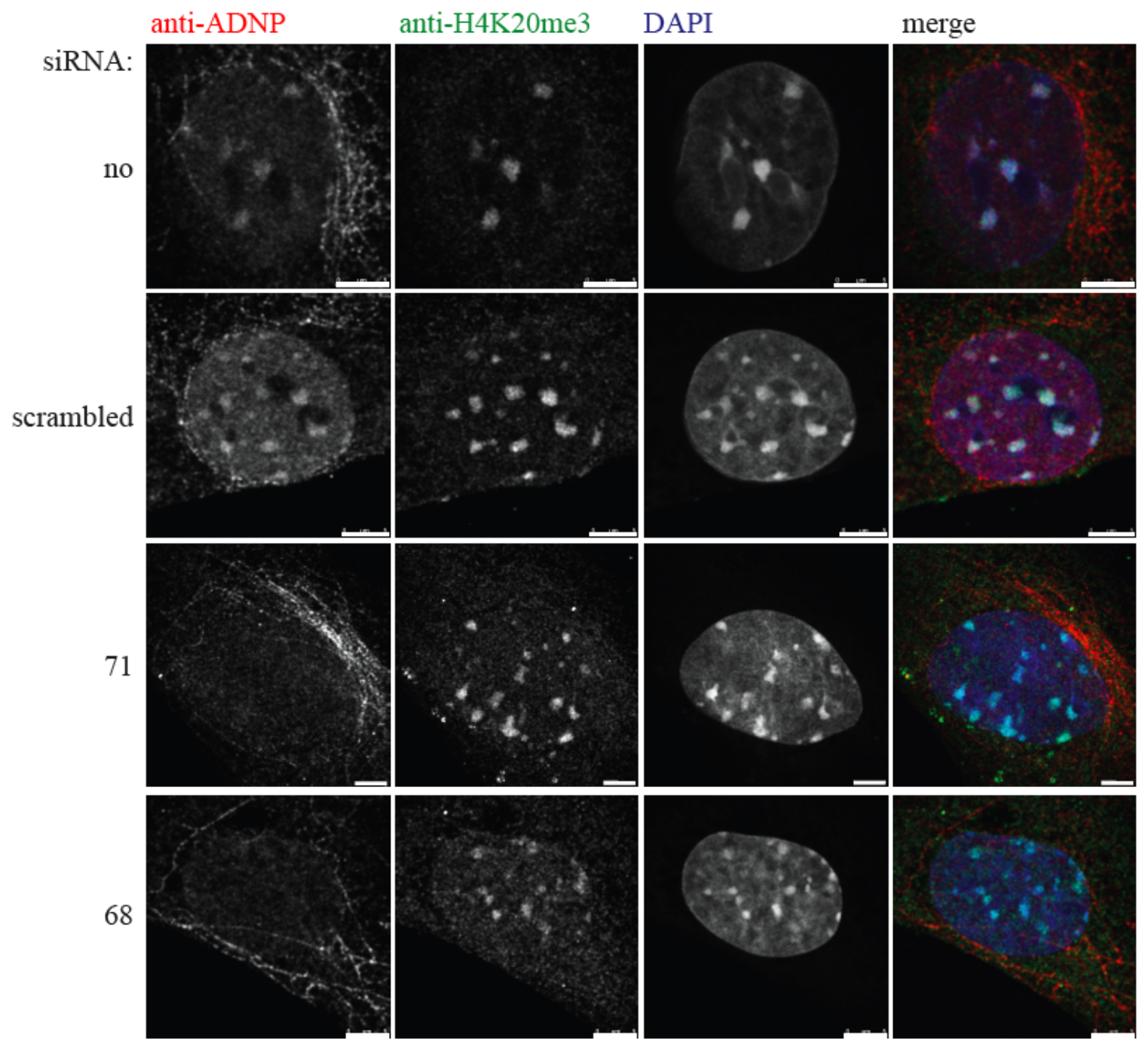

Figure 3-17: ADNP knockdown does not influence H4K20me3 distribution.

Immunofluorescence. NIH3T3, transfected with siRNA against ADNP (68 and 71), scrambled siRNA or untransfected (no) were stained with the indicated antibodies. DNA was visualized with DAPI. Bars represent $5 \mu \mathrm{m}$. 


\subsubsection{Knockdown of ADNP does not influence the global level of histone modifications}

Functional analysis of ADNP was carried out by siRNA knockdown. From a single knockdown cell preparation, I assessed the knockdown efficiency on protein level as shown in the representative Western Blot in Figure 3-18. The knockdown efficiency for all subsequent experiments is shown except for immunofluorescence analysis. A reduction of ADNP could be achieved with both siRNAs. However, the knockdown was most efficient for siRNA 71.

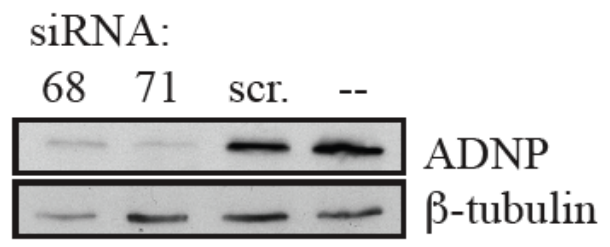

Figure 3-18: ADNP knockdown.

Western Blot. NIH3T3 cells were transfected with siRNA against ADNP (68 and 71). As controls NIH3T3 cells transfected with a scrambled siRNA and untransfected cells were used. The knockdown was verified by Western Blot using anti-ADNP and anti $\beta$-tubulin (loading control) antibodies.

Suv39h1 is a methyltransferase specific for $\mathrm{H} 3 \mathrm{~K} 9 \mathrm{me} 2 / 3$. Its knockout however does not only reduce $\mathrm{H} 3 \mathrm{~K} 9 \mathrm{me} 3$ levels but also related (H3K9me3/S10ph decrease and $\mathrm{H} 3 \mathrm{~K} 9 \mathrm{ac}$ increase) and indirectly affected (H4K20me3 decrease) histone modification levels (Schotta et al., 2004). To test if ADNP has an effect on global histone modification levels total cell extract of the ADNP knockdown NIH3T3 cells (Figure 3-18) as well as Suv39h1/h2 knockout and wild type MEF cells (positive control) was analyzed by Western Blot. Histone $\mathrm{H} 3$ and $\mathrm{H} 4$ antibodies were used as loading controls. For H3K9me3/S10ph, two different exposures are shown to detect the combined modification in the control MEF cells (upper panel) without overexposing the siRNA NIH3T3 cells 
(lower panel). The positive control MEF cells showed the expected result described above. Conversely, no effect of ADNP knockdown could be observed. For completion, H3K27me1, which is also associated with pericentromeric heterochromatin (Peters et al., 2003) was analyzed. For H3K27me1, there was no effect of Suv39 knockout and ADNP knockdown was detected.

\section{Suv39 siRNA}

$\mathrm{h} 1 / \mathrm{h} 2$

wt dn 6871 scr. --
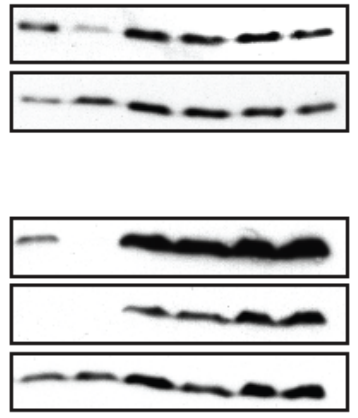

anti-H3K9me3 anti-H4

anti-H3K9me3/S10ph anti-H3K9me3/S10ph anti-H4

anti-H3K9ac anti-H4

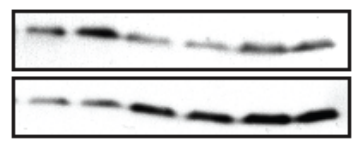

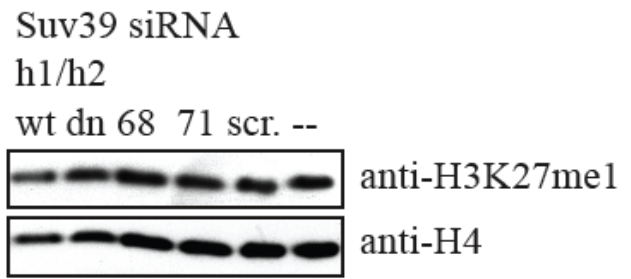

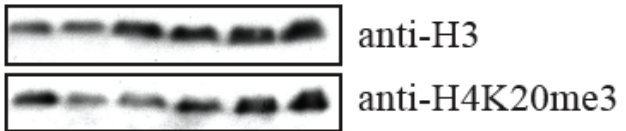

Figure 3-19: ADNP knockdown does not influence global histone modifications

Western Blot. Aliquots of the cells shown in Figure 3-18 were analyzed by Western Blotting using the indicated antibodies. The anti-H4 and anti-H3 antibodies were used to verify equal loading. Suv39h1/h2 wildtype (wt) and double knockout (dn) MEF cells served as experimental controls. 


\subsubsection{ADNP knockdown has no influence on the localization of HP1 and repressive histone modifications}

Not only the global level of modifications but also the localization of certain histone modifications, H4K20me3 and H3K27me1 as well as HP1 are affected by Suv39h1/h2 knockout (Harnicarova Horakova et al., 2009; Peters et al., 2003; Schotta et al., 2004). With my experiments, I could exclude an effect of ADNP on H3K9me3 and H4K20me3 (3.4.1). To further investigate a role of ADNP on HP1 and H3K27me1 localization, the HP1 isoforms and H3K27me1 were stained with the respective antibodies as in (3.4.1). Suv39h1/h2 knockout and wild type MEF cells were used as experimental controls. None of the HP1 isoforms localized to pericentromeric heterochromatin in the Suv30h1/h2 knockout cells whereas the distribution of HP1 remained unchanged in the ADNP knockdown cells (Figure 3-20Figure 3-21Figure 3-22). In contrast to the literature (Peters et al., 2003), I observed a mixed population of about $50 \%$ of the cells exhibiting diffuse or heterochromatic H3K27me1 signals in all MEF and NIH3T3 cells analyzed. A representative image is shown in Figure 3-23 A, upper panel. (Peters et al., 2003) described a complete loss of $\mathrm{H} 3 \mathrm{~K} 27 \mathrm{me} 1$ from pericentromeric heterochromatin. To show that Suv39h1/h2 knockout and ADNP knock-down had no such effect in my experiments, only cells showing H3K27me1 signals at pericentromeric heterochromatin are depicted. Differences in the staining intensity were within a variability also observed in the wild type cells. This variability could be seen in untransfected and scrambled siRNA transfected cells as well.

In conclusion, knock-down of ADNP had no detectable effect on HP1 and H3K27me1 distribution. 
CHAPTER 3 - Results

A

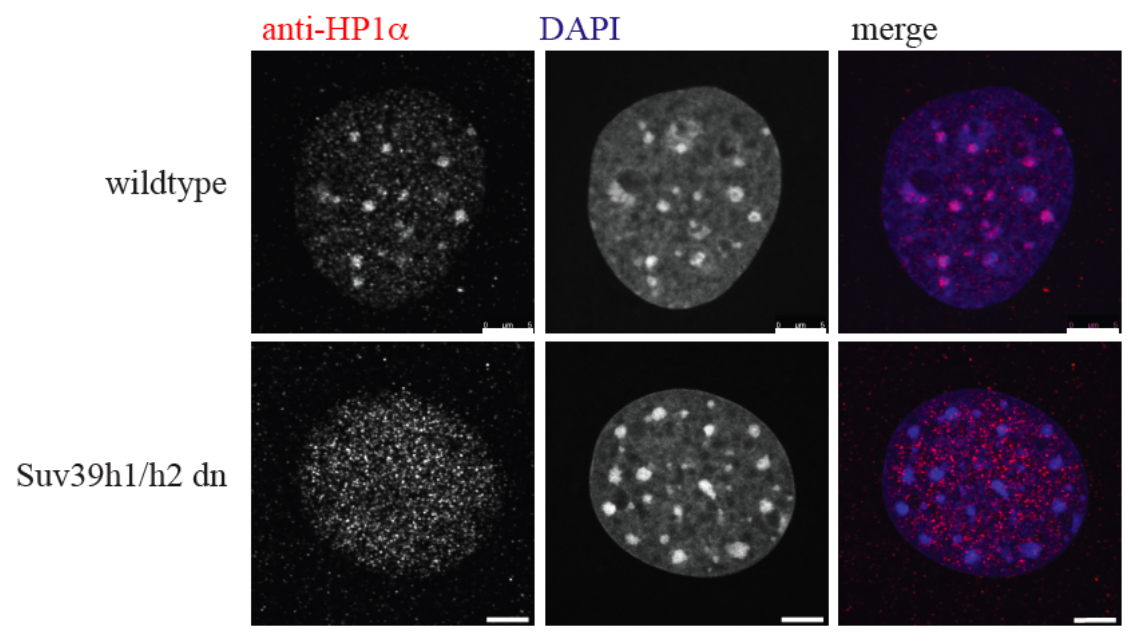

$\mathbf{B}$

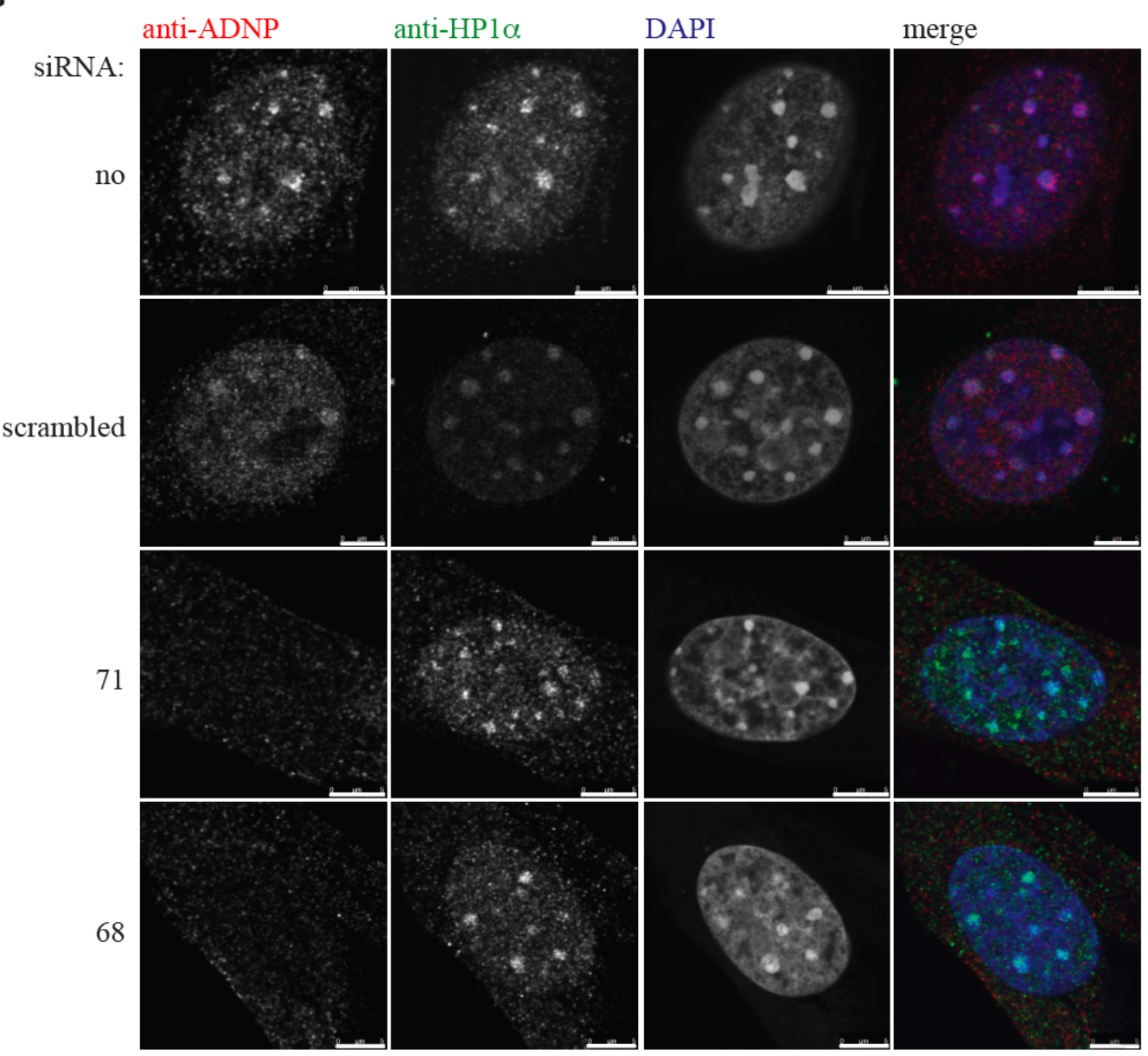

Figure 3-20: ADNP knockdown does not influence HP1 $\alpha$ localization.

Immunofluorescence. A, As an experimental control Suv39h1/h2 wildtype and double knockout (dn) MEF cells were stained with an anti-HP1 $\alpha$ antibody. B, NIH3T3 cells, transfected with siRNA against ADNP (68 and 71) scrambled siRNA or untransfected (no) were stained with the indicated antibodies. DNA was visualized with DAPI. Bars represent $5 \mu \mathrm{m}$. 
A

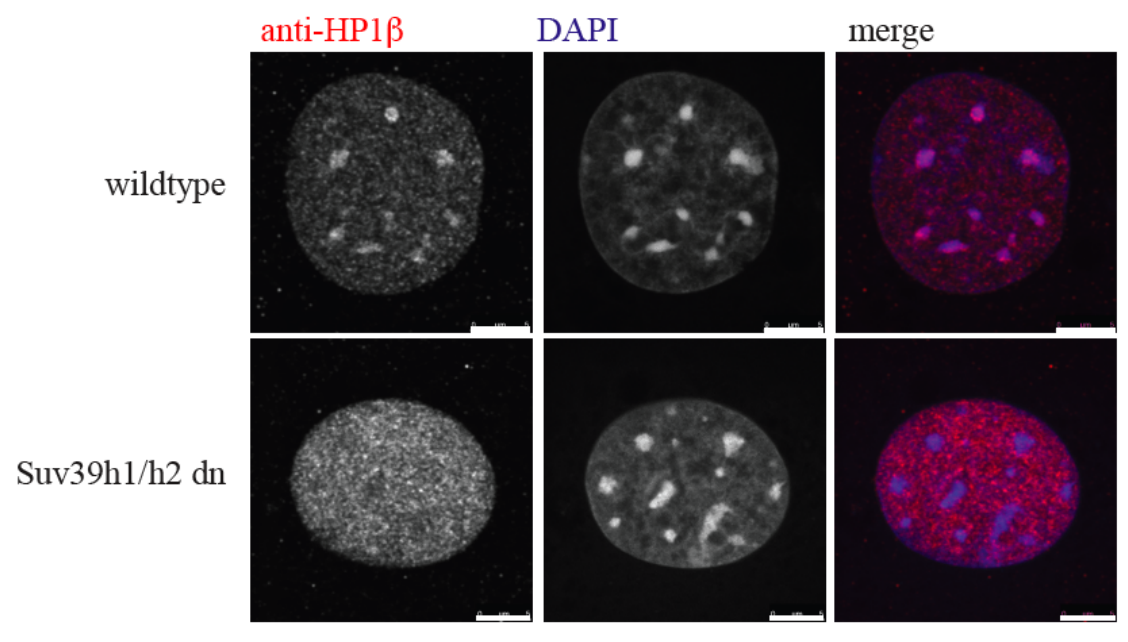

B

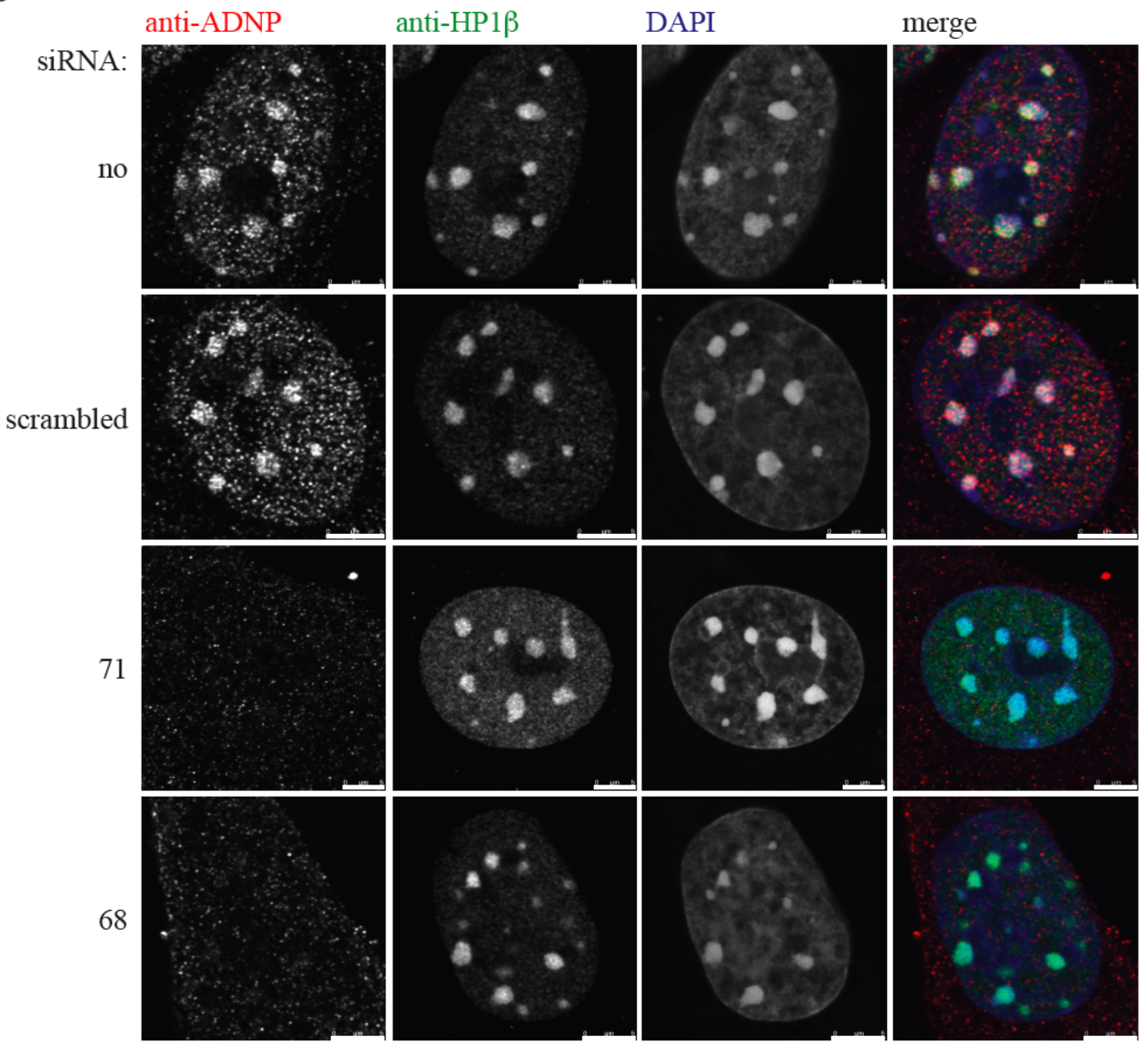

Figure 3-21: ADNP knockdown does not influence HP1 $\beta$ localization.

Immunofluorescence. A, As an experimental control Suv39h1/h2 wildtype and double knockout (dn) MEF cells were stained with an anti-HP1 $\beta$ antibody. B, NIH3T3 cells, transfected with siRNA against ADNP (68 and 71) scrambled siRNA or untransfected (no) were stained with the indicated antibodies. DNA was visualized with DAPI. Bars represent $5 \mu \mathrm{m}$. 
CHAPTER 3 - Results

$\mathbf{A}$

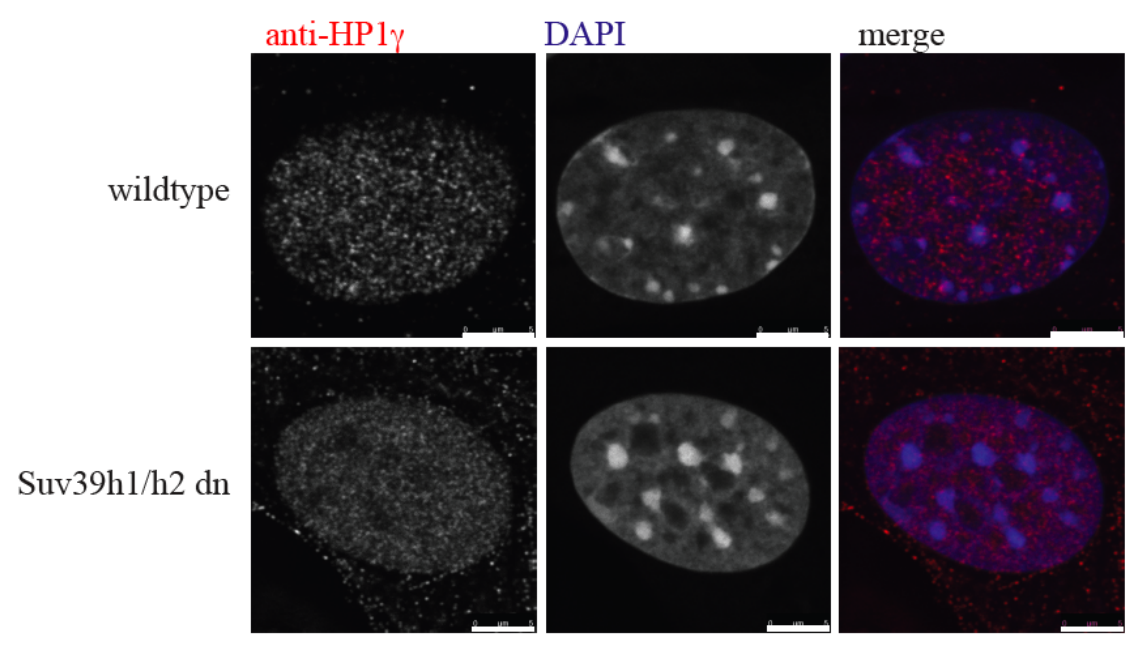

B

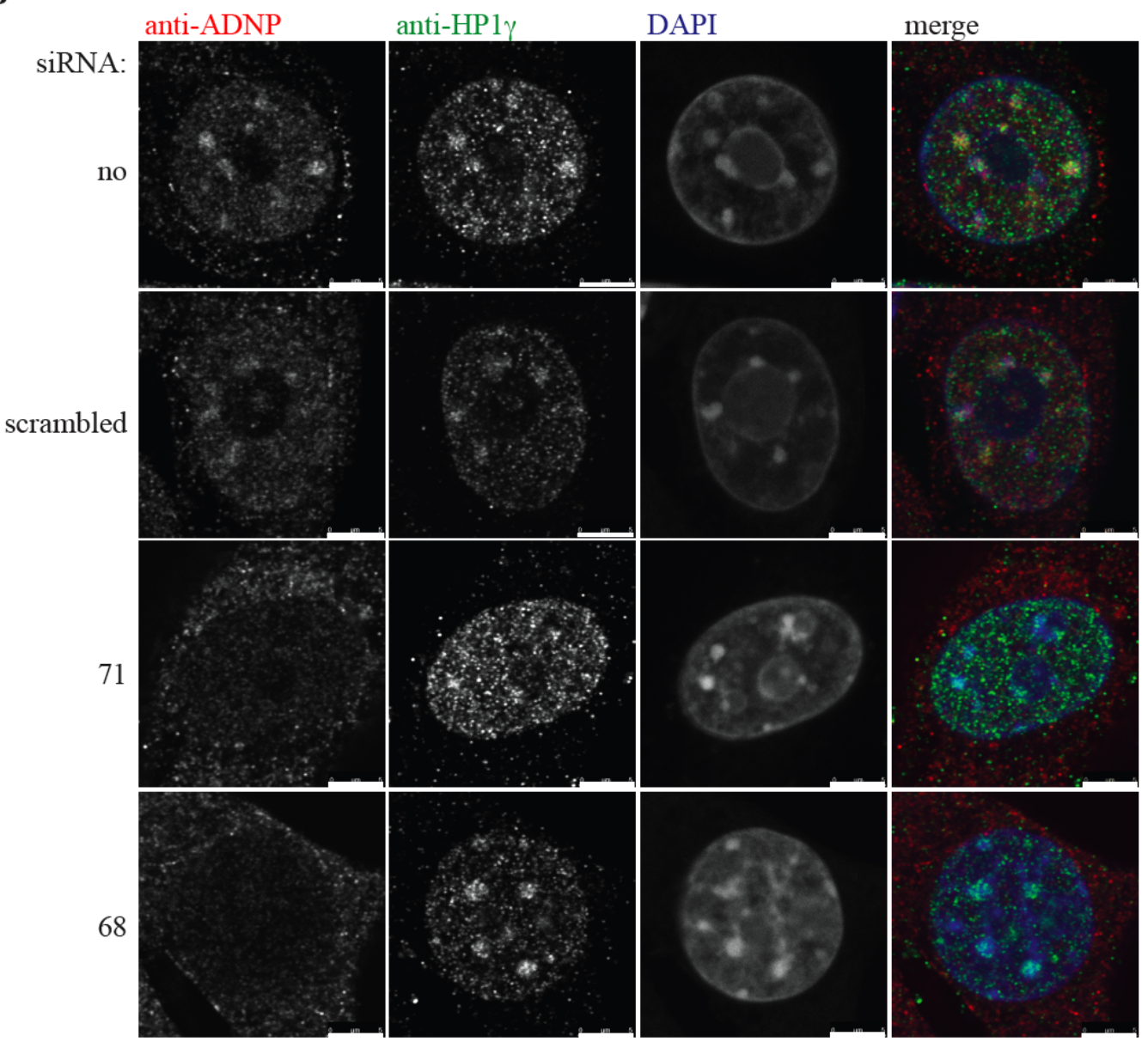

Figure 3-22: ADNP knockdown does not influence HP1 $\gamma$ localization.

Immunofluorescence. A, As an experimental control Suv39h1/h2 wildtype and double knockout (dn) MEF cells were stained with an anti-HP1 $\gamma$ antibody. B, NIH3T3 cells, transfected with siRNA against ADNP (68 and 71) scrambled siRNA or untransfected (no) were stained with the indicated antibodies. DNA was visualized with DAPI. Bars represent $5 \mu \mathrm{m}$. 
$\mathbf{A}$

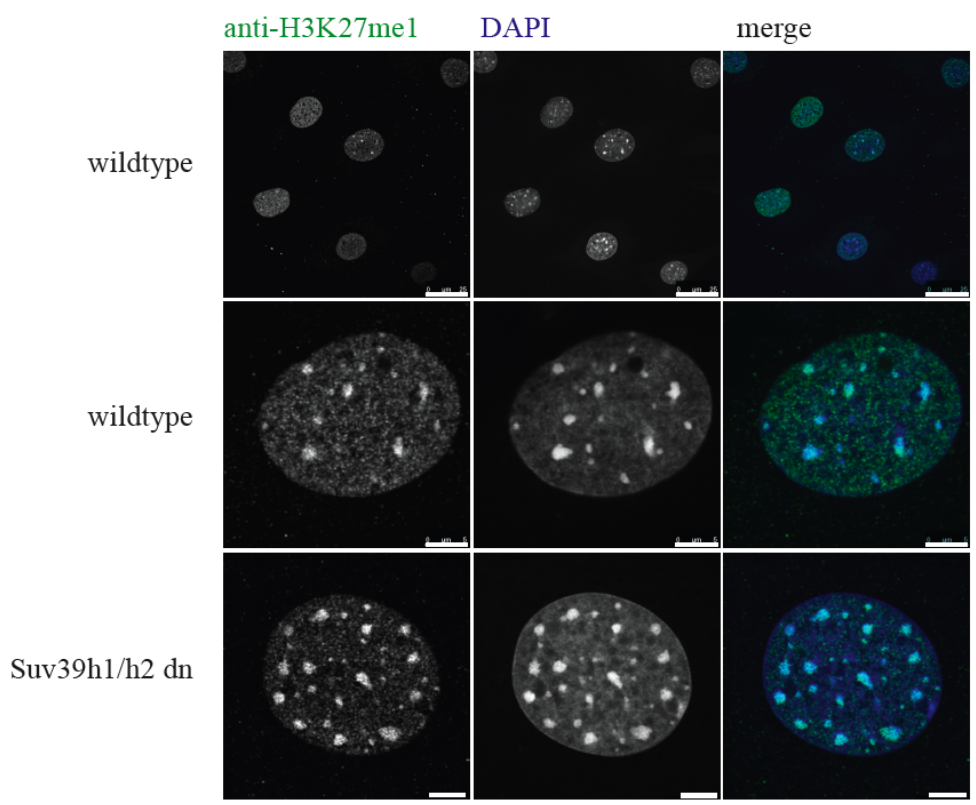

B

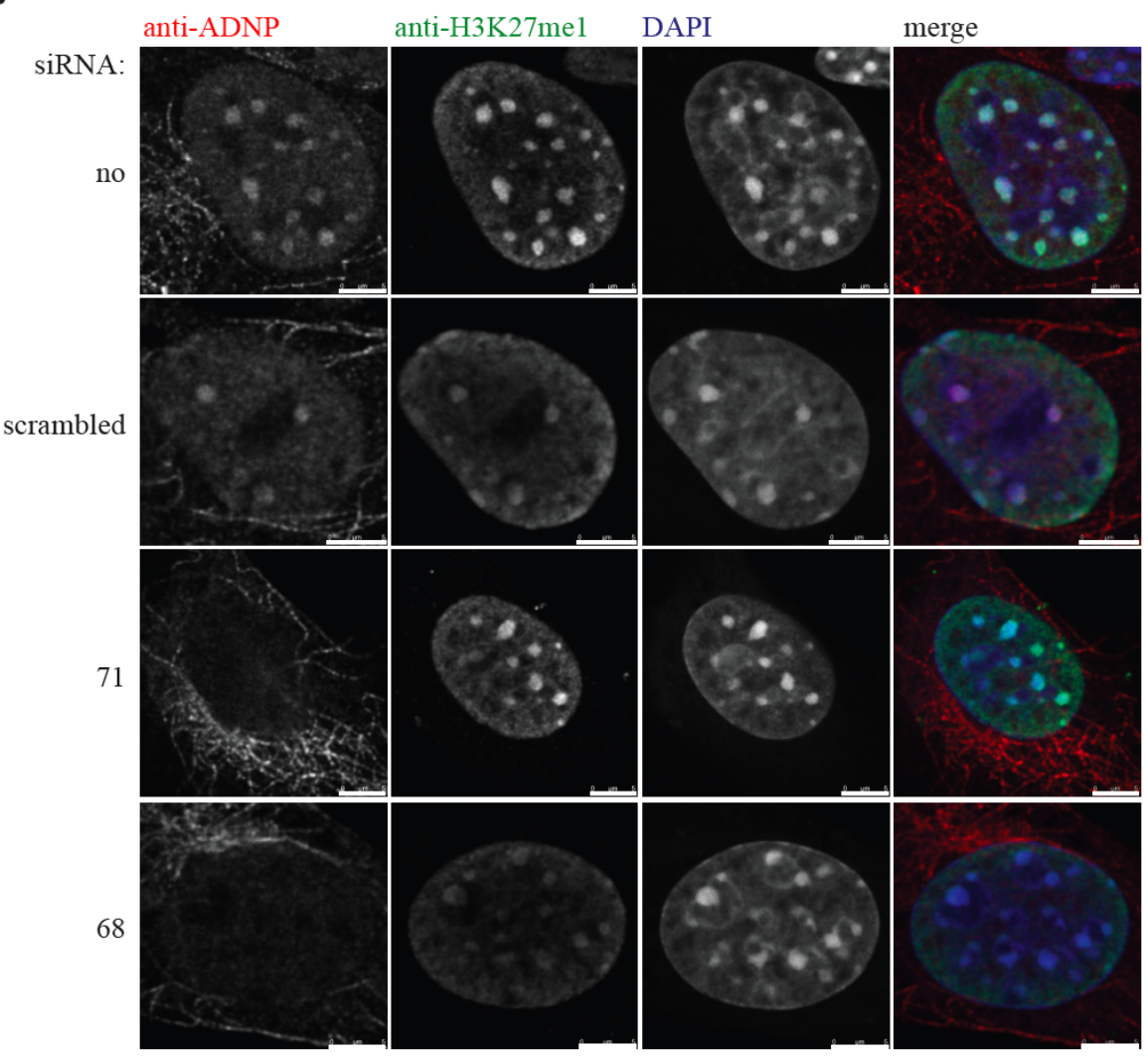

Figure 3-23: ADNP knockdown does not influence H3K27me1 distribution.

Immunofluorescence. A, As an experimental control Suv39h1/h2 wildtype and double knockout (dn) MEF cells were stained with an anti-H3K27me1 antibody. B, NIH3T3 cells, transfected with siRNA against ADNP (68 and 71) scrambled siRNA or untransfected (no) were stained with the indicated antibodies. DNA was visualized with DAPI. Bars represent $5 \mu \mathrm{m}$ and $25 \mu \mathrm{m}$ in A, upper panel. 


\subsubsection{ADNP is not involved in GpG methylation}

To determine whether ADNP is involved in the Suv39h1/h2 dependent DNA methylation at major satellite repeats, genomic DNA was extracted from ADNP knockdown and control NIH3T3 cells (Figure 3-18) as well as Suv39h1/h2 knockout and wild type MEF cells. DNA was digested with the methylation sensitive restriction enzyme Tail (5'ACGT-3') and separated on a 1\% agarose gel. By ethidium bromide staining, digested DNA could already be detected in the absence of Suv39h1/h2 indicating a global loss of CpG methylation in these cells (Figure 3-24 A). A subsequent southern blot followed by hybridization with a major satellite probe confirmed the enhanced digestion of major satellite DNA in Suv39h1/h2 knockout cells. In contrast, no enhanced digestion of global DNA or major satellite repeats was found after knockdown of ADNP (Figure 3-24).

A

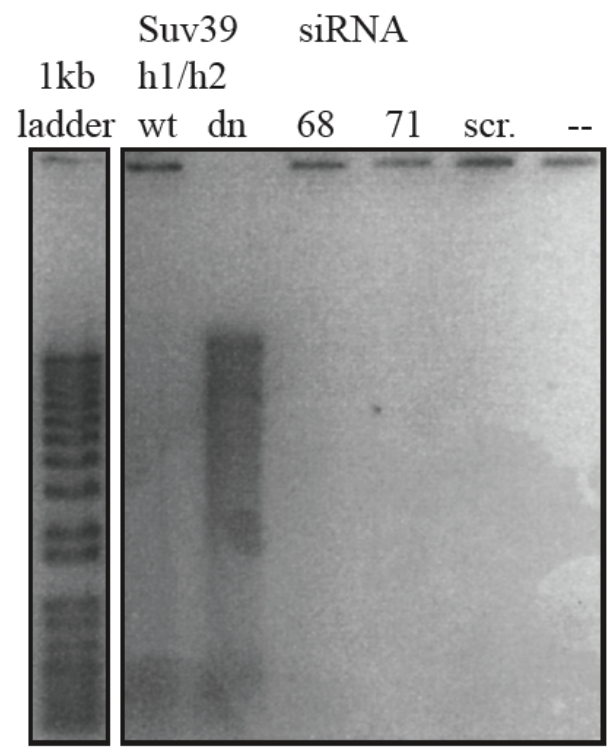

B

Suv39 siRNA

h1/h2

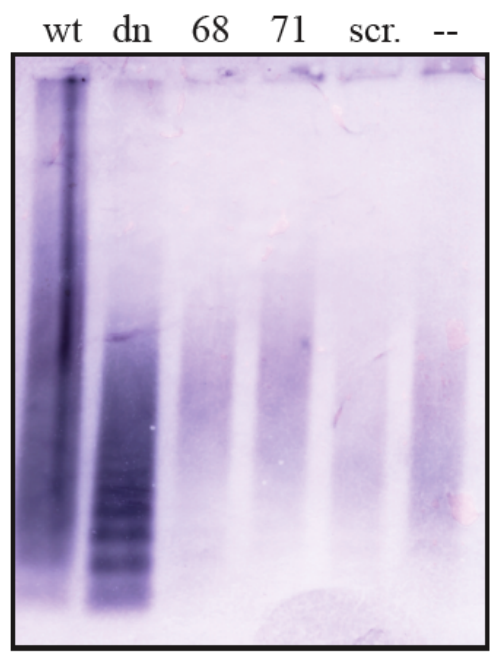

Figure 3-24: ADNP knockdown has no influence on CpG-methylation.

A and B, Genomic DNA was prepared from aliquots of the cells shown in Figure 3-19 A, digested with Tail and separated on an 1\% agarose gel. As experimental control Suv39h1/h2 wildtype (wt) and double knockout (dn) MEF cells were used. A, Ethidiumbromide staining and B, Southern blot of the agarose gel shown in A using a major satellite repeat probe are shown. 


\subsubsection{ADNP functions in silencing of major satellite repeats}

ADNP has been demonstrated to be involved in transcriptional activation as well as repression during embryogenesis (Mandel et al., 2007). To analyze whether ADNP plays a role in the Suv39h1 mediated silencing of major satellite repeats (Lehnertz et al., 2003) I addressed the activating or repressive potential of ADNP. I applied a luciferase reporter assay where the firefly luciferase is under the control of a weak promoter (TK-promoter). This setup allows the detection of enhanced as well as reduced transcription depending on the activity of the protein that is targeted to the UAS in front of the promoter (Figure 3-25 A). I used this construct either after transient transfection into HEK 293 cells or stably integrated and thereby chromatinized in HEK 293 cells (HEK TK22) (Ishizuka and Lazar, 2003). Gal4 tagged ADNP was transfected into these cells together with a plasmid containing the renilla luciferase gene controlled by the CMV promoter to measure the transfection efficiency. The transcriptional repressor (SMRT) fused to Gal4 served as an experimental control. Luciferase expression levels were measured by substrate turnover, which results in luminosity. Firefly luciferase luminosity was first normalized to the renilla luciferase signal and second to the untransfected control cells.

Transfection of Gal4-SMRT strongly reduced the firefly luciferase signal in a dosedependent manner. Although the reduction in luminosity after transfection of increasing amounts of Gal4-ADNP was not as strong as that of Gal4-SMRT a silencing activity of ADNP was clearly observed in both cell lines after transient transfection of the reporter gene cassette or in a stably integrated, chromatinized context. 
A

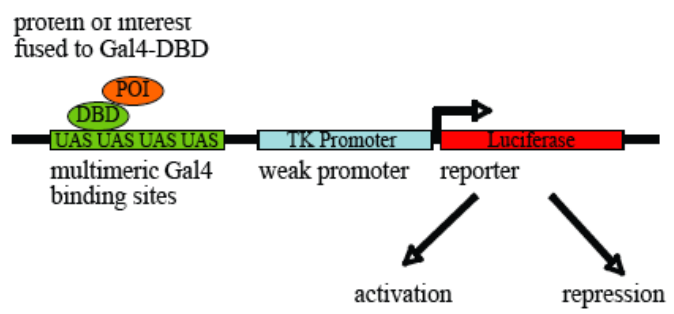

B

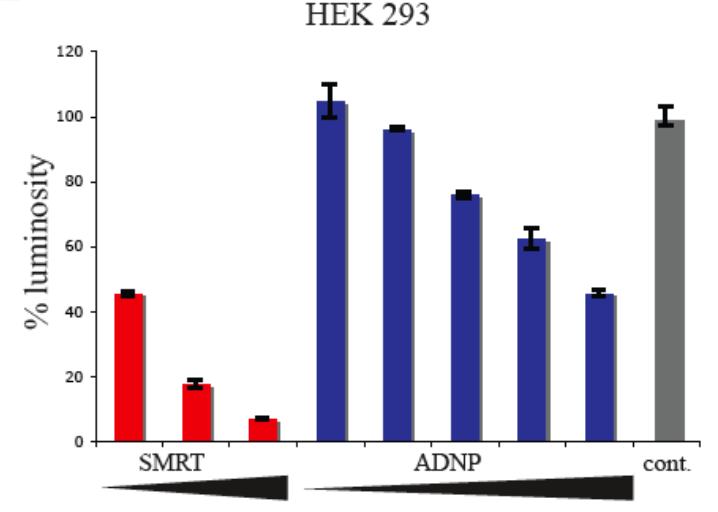

$\mathrm{C}$

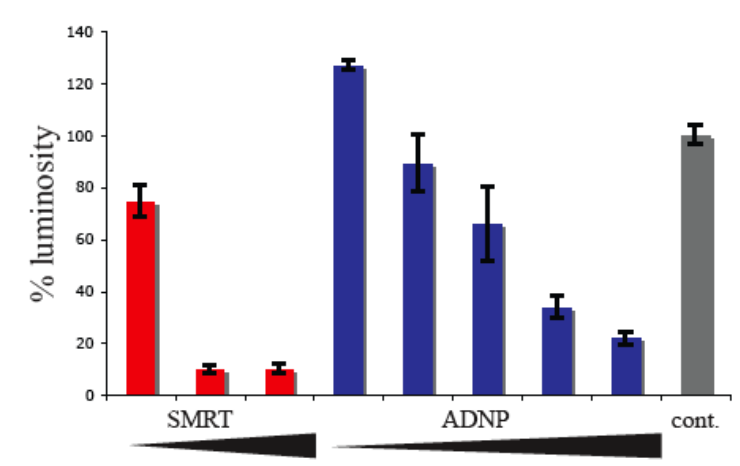

Figure 3-25: ADNP has silencing activity.

A, Schematic representation of the construct used for the luciferase reporter assay. The protein of interest (POI) is fused to the Gal4-DNA binding domain (DBD) and thus recruited to the Gal4-binding site (UAS) upstream of the TK-promoter. The TK-promoter allows a basal transcription of the Firefly-luciferase, which is used as a reporter. Depending on the properties of the POI, luciferase expression can be up or down regulated. B, The reporter construct was transiently transfected or C, stably integrated in HEK 293 cells. Increasing concentrations of SMRT (positive control) or ADNP were transfected. pCDNA3.1 was used as control. Luciferase activity was measured 1dpt (HEK 293) or 2dpt (HEK TK22).

To test whether ADNP has a silencing function at pericentromeric heterochromatin I determined the transcriptional status of major satellite repeats. I performed RT-PCR analyses on total RNA that had been reverse transcribed with random oligo-dT primers. Using specific primers the abundance of major satellite transcripts relative to GAPDH mRNA was determined by real-time PCR. The relative major satellite expression is displayed in Figure 3-26 as the $2^{- \text {ct }}$ value. The Suv39h1/h2 knockout as well as the HP $1 \alpha / \beta$ knockout cells showed a clear increase in major satellite transcription compared to the respective wild type MEFs. In the ADNP knockdown cells the level of these transcripts was only elevated after treatment with siRNA 71 but not with siRNA 68 . To 
analyze whether this is a consequence of the stronger reduction of ADNP with siRNA 71 or is due to an off-target effect of this particular siRNA I repeated the experiment with two YFP-ADNP or YFP overexpressing cell-lines. ADNP expression levels of these cells were analyzed by Western Blot. Compared to the YFP expressing controls YFP-ADNP overexpression reduced major satellite expression significantly. (Figure 3-26)
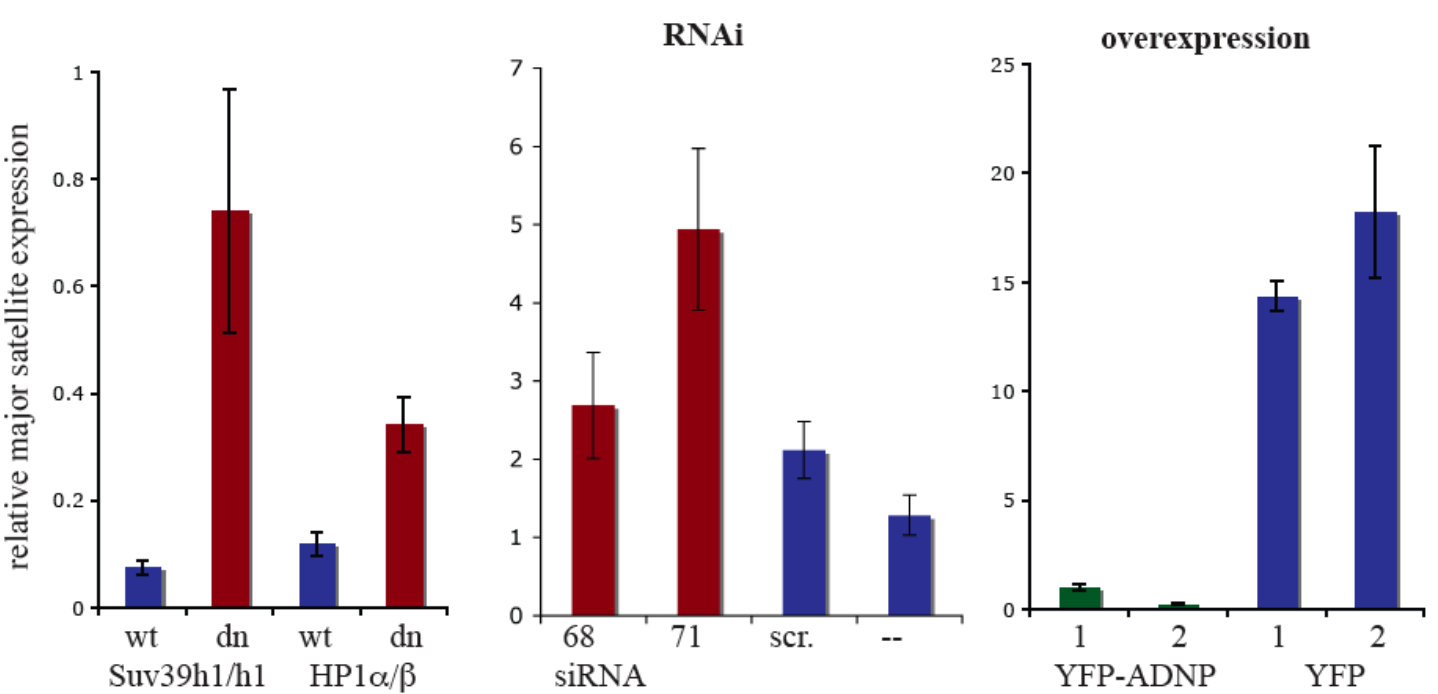

Figure 3-26: ADNP influence on major satellite repeat expression

A, Western Blot. ADNP expression levels of two YFP-ADNP and YFP (control) overexpressing cell lines were analyzed by Western Blot. The upper band represents YFP-ADNP, the lower band the endogenous protein. Tubulin served as loading control. B, quantitative RT-PCR. Total RNA was extracted from Suv39h1/h2 and HP1 $\alpha / \beta$ double knockout MEF (red), ADNP knockdown (red) and YFP-ADNP overexpressing (green) NIH3T3 cells. The respective wild type and YFP cells served as control (blue). Reverse transcription was followed by real-time PCR. Relative major satellite expression, normalized to GAPDH is shown. Error bars represent the standard deviation of a triplicate experiment.

Summing up, analysis of ADNP knockdown in NIH3T3 cells did not indicate an ADNP function in Brg1 mediated organization of pericentromeric heterochromatin, Also, effects on histone modifications, HP1 distribution and DNA methylation as observed in the 
Suv39h1/h2 knockout were not detected. Although up-regulation of major satellite transcription was only detected with one of the two siRNAs, additional results from luciferase assays and ADNP overexpression suggest that ADNP is involved in transcriptional silencing at pericentromeric heterochromatin. 


\section{Discussion}

\subsection{Identification of $\mathrm{H} 3 \mathrm{~K} 9 \mathrm{me} 3$ associated proteins}

A scientific challenge in chromatin research is trying to understand how factors and enzymes that set or recognize histone modifications and other factors/components interplay to establish and maintain distinct functional domains of chromatin in higher eukaryotes. Genetic approaches have identified sets of proteins, e.g. suppressors and enhancers of variegation ( $\mathrm{Su}(\mathrm{var})$ and $\mathrm{E}(\mathrm{var})$ ) that led to first insights into chromatin organization pathways. Recent advances in mass spectrometry introduced proteomic techniques to compile a list of factors that is necessary (and sufficient) to mediate distinct states of chromatin. One such method to discover chromatin associated factors is a pulldown assay using histone tail peptides modified at distinct residues (Wysocka et al., 2005). However proteins that require chromatin as a substrate for interaction may not bind peptides immobilized on beads. Using oligonucleosomes instead of peptides facilitates binding of factors that depend on additional contacts with histones, DNA or chromatin structure. A single amino acid modification such as trimethylation of histone H3 lysine 9 used in this study may cause a change in affinity for that site rather than a completely bound or unbound state of some proteins. To detect such quantitative differences stable isotope labeling by amino acids in cell culture (SILAC) was used. These differences are displayed as the ratio between the isotope labeled (heavy) and unlabeled (light) form of the proteins in the samples, in this case pulldowns of H3K9me3 or unmodified oligonucleosomes respectively. To exclude differences resulting from the heavy and light nuclear extracts a reversed biological replicate was conducted (light extract for H3K9me3 and heavy extract for unmodified oligonucleosomes). Most proteins have a ratio between 0.5 and 2, indicating no enrichment in both samples. Such proteins bind either to chromatin independent of the histone methylation state or unspecifically to the beads. The overlap of proteins enriched at least 2-fold at H3K9me3 in replicate experiments consisted of only 20 proteins. Among these proteins were known 
heterochromatin factors such as all isoforms of HP1, UHRF1/2 as well as POGZ, which was recently described to interact with HP1 (Brasher et al., 2000; Karagianni et al., 2008; Nozawa et al., 2010). One of the factors, DNA methyltransferase 1 (DNMT1) was indeed only identified in the oligonucleosome but not in the peptide context (Franz et al., 2009). This indicates specificity of the method. However, the total number of proteins was not higher than that of the peptide pulldown method. Possibly, binding of larger complexes or additional factors requires not only one modification for interaction but rather a combination of different heterochromatin marks such as histone modifications and DNA methylation. Another reason for the low number of factors found in the chromatin pulldown experiment might be, that the dodecameric oligonucleosomes are too short to form higher order structures resembling those within a cell nucleus. Therefore, the structures or the local density of the $\mathrm{H} 3 \mathrm{~K} 9 \mathrm{me} 3$ mark required for binding of larger complexes could not be achieved with this experimental setup.

\subsection{ADNP is a novel component of pericentromeric heterochromatin}

ADNP was originally discovered as a gene product associated with neuroprotection/neuroglia interactions (Bassan et al., 1999). In the nucleus, it has been shown to be involved in transcriptional regulation of genes that are associated with e.g. lipid metabolism and organogenesis (Mandel et al., 2007). ADNP has also been identified as an interaction partner of the SWI/SNF chromatin-remodeling complex. Association of ADNP with specific histone modifications and pericentromeric heterochromatin had not been described before. Therefore, I decided to focus my thesis work on characterizing the properties and functions of this protein in relation to heterochromatin.

The identification of ADNP as an $\mathrm{H} 3 \mathrm{~K} 9 \mathrm{me} 3$ associated factor by mass spectrometry was verified by Western-blot (Figure 3-3). In vivo, H3K9me3 is enriched at pericentromeric heterochromatin that can be visualized in mouse cells as DAPI-dense regions. Antibody staining of ADNP revealed that ADNP co-localizes with $\mathrm{H} 3 \mathrm{~K} 9 \mathrm{me} 3$ at heterochromatin in 
mouse fibroblasts (Figure 3-4). A speckled staining in the nucleus and a tubulin-like staining in the cytoplasm were also observed. Ectopic expression of YFP-ADNP results in a diffuse signal within the nucleus and enrichment at DAPI-dense regions (Figure 3-8). These results suggests that ADNP is localized to pericentromeric heterochromatin but is not restricted to these areas. Like other heterochromatin associated factors such as HP1 and CDYL, ADNP is displaced from chromatin during mitosis. The majority of nucleosomes containing histone $\mathrm{H} 3$ methylated on lysine 9 are then phosphorylated on serine 10, a double-modification that evicts HP1 from mitotic chromatin. However, a small number of $\mathrm{H} 3$ nucleosomes at centromeric/pericentromeric regions still bind HP1 $\alpha$ and these might serve as the foundation or could regulate the association of the KMN network (the structural core of the kinetochore, named after its constituents: the protein KNL-1/Spc105/Blinkin, and the Mis12 and the Ndc80 subcomplexes) with the centromere (Przewloca and Glower, 2009). The behavior of ADNP in that context could not be determined because of the cross-reactivity of the antibody with tubulin-like structures (Figure 3-5). The cytoplasmic staining is in contrast to reports that ADNP is excluded from the cytoplasm in fibroblasts (Divinski et al., 2006). Subcellular fractionation and expression of YFP-ADNP did not show cytosolic signals (Figure 3-4, Figure 3-8). Therefore, I consider the cytoplasmic staining as an artifact of unspecific binding of the anti-ADNP antibody.

Next, I investigated whether ADNP requires modified chromatin context instead of H3K9me3 peptides for interaction. The oligonucleosomes used for the pull-down are identical except for the methylation of histone $\mathrm{H} 3$ lysine 9, suggesting that the specificity is conferred by this modification. Protein structure predictions however show that ADNP consists of zinc-fingers and a homeobox, which are domains involved in DNA binding. ADNP chromatin immunoprecipitation showed interaction with multiple gene promoters, which confirm DNA binding capability of ADNP (Mandel et al., 2007). There are two possible hypothesis how DNA binding could be involved in ADNP association with heterochromatin: unspecific DNA binding might be necessary to stabilize ADNP at chromatin after recruitment by $\mathrm{H} 3 \mathrm{~K} 9 \mathrm{me} 3$ or specific DNA and $\mathrm{H} 3 \mathrm{~K} 9 \mathrm{me} 3$ binding may act synergistically to recruit ADNP to pericentromeric heterochromatin. For the 
chromatin pull-down an artificial nucleosome positioning sequence was used (Lowary and Widom, 1998; Thastrom et al., 1999). In this experimental setting only unspecific DNA binding might play a role. To test this a peptide pulldown was performed. Here only unmodified and methylated histone tail peptides were used. ADNP association with the H3K9me3 peptide bound beads excludes that DNA binding is needed for recruitment to this modification in vitro. However, a weak affinity of ADNP for DNA might stabilize the binding to chromatin after recruitment. The specific binding of DNA at pericentromeric heterochromatin cannot be excluded. However, in the Suv39h1/h2 double knockout cells where $\mathrm{H} 3 \mathrm{~K} 9 \mathrm{me} 3$ is not enriched at pericentromeric heterochromatin, ADNP is displaced from these areas as well (Figure 3-6). If specific DNA binding occurs at pericentromeric heterochromatin, it is not sufficient for ADNP recruitment.

\subsection{ADNP is targeted to pericentromeric heterochromatin by HP1}

I observed that ADNP localizes to pericentromeric heterochromatin. How is ADNP then targeted to these regions? Structural prediction algorithms did not detect known methyllysine binding domains such as a chromo domain, a plant homeo domain, a tudor domain or a malignant brain tumor domain. The only domains predicted in ADNP are a homeodomain and zinc fingers. For the majority of the 1108 amino acids protein the structure is completely unknown. Still, the possibility that ADNP binds directly to H3K9me3 by a domain that lacks sufficient homology for structural prediction can not be excluded by computational analysis. However, in vitro results from peptide pulldowns using ADNP expressed in reticulocyte lysate showed no binding of ADNP to H3K9me3 peptides (Figure 3-9). Immunofluorescence analysis in HP1 $\alpha / \beta$ knockout cells where the level and distribution of $\mathrm{H} 3 \mathrm{~K} 9 \mathrm{me} 3$ remained unaffected revealed delocalization of ADNP from chromocenters in these cells (Figure 3-10). These results suggest that ADNP is not recruited to pericentromeric heterochromatin by direct binding to $\mathrm{H} 3 \mathrm{~K} 9 \mathrm{me} 3$. 
A likely candidate for indirect ADNP recruitment is HP1. During these studies the interaction of ADNP with HP1 $\alpha$ was suggested (Mandel et al., 2007). The ADNP homeodomain contains a PxVxL and an ARKS motif. PxVxL motifs in general are binding motifs of the HP1 chromoshadow domain (Thiru et al., 2004). ARKS is a motif in the histone $\mathrm{H} 3$ tail that encompasses of lysines 9 and 27. When lysine 9 of histone $\mathrm{H} 3$ is trimethylated it is recognized by the HP1 chromo domain. Trimethylated lysine 27 is also bound by HP1 but with a lower affinity. ADNP has also been shown to interact with the SWI/SNF complex via interaction of Brg1 most likely with the C-terminus of ADNP (Mandel and Gozes, 2007). Although Brg1 does not localize to DAPI dense regions, its knockout causes misorganization of pericentromeric heterochromatin (Bourgo et al., 2009). In ADNP deletion mutants the homeodomain was necessary and sufficient for targeting to heterochromatin (Figure 3-8). This result and the delocalization of ADNP in the HP1 $\alpha / \beta$ knockout cells indicate that HP1 is the main mediator of ADNP heterochromatin recruitment. Interestingly, knockout of single HP1 isoforms did not affect ADNP localization, suggesting that ADNP interaction with HP1 is not limited to a particular isoform (Figure 3-10). Peptide pulldown experiments confirmed that all isoforms of HP1 are indeed able to target ADNP to H3K9me3 peptides (Figure 3-9).

\subsection{Mapping of the HP1 - ADNP interaction interface}

To further map the HP1 - ADNP interaction point mutants were introduced into the ARKS and the PxVxL motifs. A lysine to arginine mutation in the ARKS motif prevents its putative trimethylation. This mutation in a histone $\mathrm{H} 3$ peptide context has been described to prevent HP1 binding. Therefore I introduced this mutation into the ADNP ARKS sequence (K767R). For the PxVxL motif a valine to glutamate mutation has been shown to abolish the interaction with HP1. Thus, valine 821 in the ADNP homeodomain was mutated to glutamate (V821A). Live cell imaging as well as peptide pulldowns showed no effect of the K767R mutation on ADNP targeting. In contrast, the V821A mutation resulted in ADNP highly reduced signals at DAPI dense regions and $\mathrm{H} 3 \mathrm{~K} 9 \mathrm{me} 3$

peptides in these experiments. Interestingly, only in the K767R/V821E double mutant 
recruitment to pericentromeric heterochromatin and $\mathrm{H} 3 \mathrm{~K} 9 \mathrm{me} 3$ peptides was completely abolished (Figure 3-12). In agreement with these results, W170A mutation in the HP1 chromoshadow domain, which has no influence on $\mathrm{H} 3 \mathrm{~K} 9 \mathrm{me} 3$ binding and HP1 dimerization but eliminates $\mathrm{PxVxL}$ binding, strongly reduced ADNP recruitment to H3K9me3 (Figure 3-14). Obviously, HP1 binding and subsequent targeting of ADNP mainly depends on the PxVxL motif. Nevertheless, an additional effect of the K767R mutation was detected. This effect could be explained by (i) an influence on ADNP dimerization/multimerization preventing recruitment of the non HP1 bound ADNP mutant by endogenous ADNP, (ii) stabilization of the HP1 binding to the PxVxL motif by synergistically binding to a hypothetical ADNP K767me3 or (iii) disruption of the homeodomain structure. Hypothesis (i) was excluded by endogenous ADNP coprecipitating with wild type and all point mutants of YFP-ADNP (Figure 3-13). To address possibility (ii) I tested whether ADNP can be methylated and whether K767me3 would be bound by HP1. The result shown in Figure 3-15 C suggests that ADNP might indeed be methylated. However, in vitro HP1 did not bind to ADNPK767me3 peptides (Figure 3-15 D). A potential reason for that behavior lies in the residues surrounding the ARKS motif. The structure of a histone tail bound HP1 chromo domain has been published. If the threonine preceding the ARKS in the H3 sequence is replaced by a glutamate as in the ADNP sequence, a steric inhibition of peptide binding seems likely (Figure 4-1). 
$\mathbf{A}$

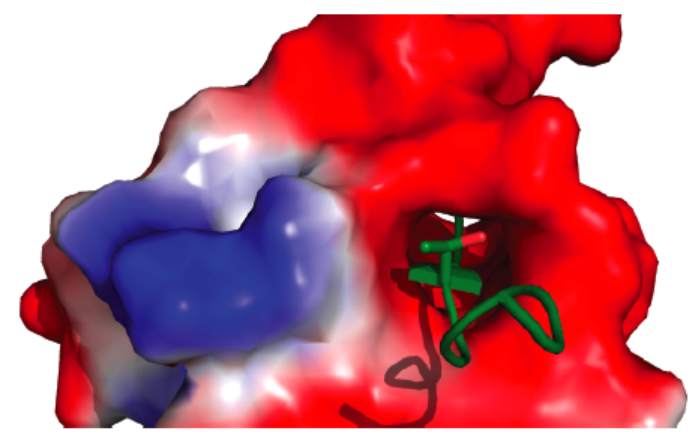

ART Kme2 QTAR Kme2 STGGKAPGG
B

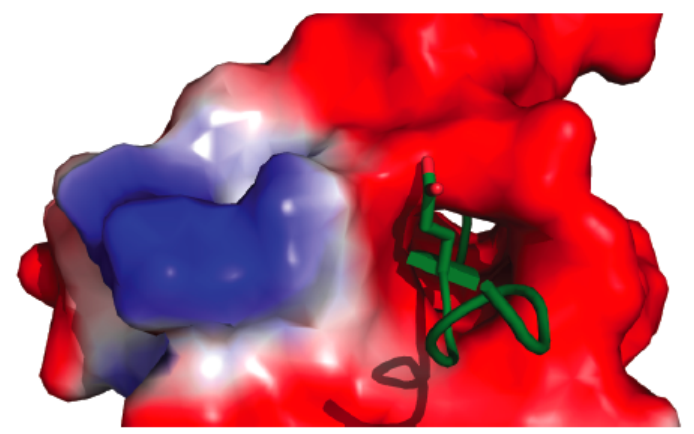

ART Kme2 QEAR Kme2 STGGKAPGG

Figure 4-1: Structure of mouse HP1 $\beta$ chromo domain.

A. Crystal structure of mouse HP1 $\beta$ chromo domain in complex with the lysine 9 methyl H3 N-terminal peptide (1GUW) as a cartoon. The surface charge is represented ranging from red (acidic) to blue (basic). The histone tail peptide is schown as sticks. B. Threonine 6 was replaced by a glutamate as in the ADNP sequence in silico (Nielsen et al., 2002).

The remaining hypothesis is that K767R causes disruption of the ADNP homeodomain structure. Since HP1 has been shown to bind several different proteins having different domains / structures via their PxVxL motif, this type of interaction seems not to require a certain structure. Any remaining PxVxL independent interaction that might be mediated by the homeodomain could be abolished by the K767R mutation.

\subsection{Nuclear function of ADNP}

Complete deficiency of ADNP in mouse embryos results in dramatic changes in gene expression (Mandel et al., 2007). Up-regulated transcripts were clustered into a family encoding proteins enriched in the visceral endoderm such as apolipoproteins, cathepsins and methallotionins. In contrast, a down-regulated gene cluster consisted of organogenesis markers including neurogenesis and heart development (Mandel et al., 2007). ADNP functions at pericentromeric heterochromatin have not yet been described. 
Interaction between ADNP and three members of the SWI/SNF chromatin-remodeling complex (BAF250a, BAF170 and Brg1) has been shown by co-immunoprecipitation (Mandel and Gozes, 2007). Although Brg1 does not localize to DAPI dense regions its deficiency results in dissolution of pericentromeric heterochromatin and delocalization of the heterochromatin markers H3K9me3 and H4K20me3 (Bourgo et al., 2009). ADNP could be involved in that process by recruiting Brg1 or by modulating the activity of the SWI/SNF complex. By immunofluorescence analysis however, no effect of ADNP knockdown on the integrity of chromocenters and the distribution of trimethylated H3K9 and H4K20 were detected (Figure 3-16; Figure 3-17).

Since ADNP loss of function phenotypes should also be observed in Suv39h1/h2 knockout cells, where HP1 and ADNP are delocalized from pericentromeric heterochromatin (Figure 3-6; Figure 3-20; Figure 3-21; Figure 3-22), I tested ADNP knockdown cells for several described Suv39h1/h2 knockout phenotypes.

Suv39h1/h2 are methyltransferases specific for lysine 9 of histone H3 at pericentromeric heterochromatin. Therefore, its depletion results in a reduction of $\mathrm{H} 3 \mathrm{~K} 9 \mathrm{me} 3$ and the loss of this modification from pericentric heterochromatin. Reduction of H3K9me3 levels leads to a decrease of combination $\mathrm{H} 3 \mathrm{~K} 9 \mathrm{me} 3$ with serine 10 phosphorylation and an increase of lysine 9 acetylation. These are primary effects of the Suv39h1/h2 knockout. However, ADNP might be involved in Suv39h1/h2 targeting to or stabilization at heterochromatin or it may influence Suv39h1/h2 enzymatic activity. Another heterochromatin marker, H4K20me3 is also mislocalized after loss of Suv39h.1/h2 H4K20me3 has been proposed to be mediated by H3K9me3-bound HP1 recruiting Suv420, the H4K20 methyltransferase (Schotta et al., 2004). Since ADNP interacts with HP1, an influence on that process seems plausible. A reported loss of H3K27 monomethlyation in Suv39h1/h2 knockout cells (Peters et al., 2003) could neither be reproduced with a commercial antibody nor with the antibody used in the original report. Immunofluorescence and Western Blot analysis however did not show any differences in the described histone modifications (Figure 3-16; Figure 3-17; Figure 3-19; Figure 3-23). 
Both histone modifications and HP1 are displaced from pericentromeric heterochromatin in Suv30h1/h2 knockout cells (Harnicarova Horakova et al., 2009) This result is most likely an effect of the loss of H3K9me3. Nevertheless ADNP might mediate the specificity of HP1 $\alpha$ and/or HP1 $\beta$ for these areas. In vitro, no specificity of ADNP for a certain HP1 isoform was detected (Figure 3-9). Consistently ADNP knockdown did not result in relocalization of HP1.

DNA methylation at major satellite repeats has been reported to occur either by the DNA methyltransferase DNMT1 in a Suv39h1/h2 independent manner (Chuang et al., 1997; Rountree et al., 2000) or by DNMT3a/b dependent on Suv39h1/h2 (Lehnertz et al., 2003). DNMT3a and DNMT3b were shown to interact with HP1 $\alpha$ and HP1 (Lehnertz et al., 2003), raising the possibility that ADNP might play a role in that process. Digestion with a methylation sensitive restriction enzyme, however, did not reveal any influence of ADNP knockdown on global DNA methylation or DNA methylation of major satellite repeats.

Finally, Suv39h1/h2 have been reported to be involved in transcriptional silencing of major satellite repeats (Lehnertz et al., 2003). Depletion of Suv39h1/h2 results in loss of markers for transcriptional repression such as histone modifications, DNA methylation and HP1 localization (as described in the previous section) yet the exact mechanism to reach a silent state remains unknown. In Suv39h1/h2 as well as in HP1 $\alpha / \beta$ knockout cells transcription of major satellite repeats was increased. Enhanced major satellite repeat transcription was also observed after transfection with one of the two siRNAs (siRNA 71) against ADNP (Figure 3-26). siRNA 71 induced a stronger reduction of ADNP than siRNA 68 ( $\sim 85 \%$ vs. $\sim 60 \%$ of scrambled siRNA control) (Figure $3-18)$. The knockdown efficiency of siRNA 68 might not be sufficient to cause an effect on transcription. On the other hand, the enhanced transcription after transfection of siRNA 71 could be due to an off-target effect of that particular siRNA. Additional results from luciferase assays and YFP-ADNP overexpression however indicate, that ADNP is indeed involved in silencing of major satellite repeats (Figure 3-25,Figure 3-26). 
These results lead to the proposal of the following model for ADNP recruitment to and function at pericentromeric heterochromatin: Histone H3 is trimethylated at lysine 9 by Suv39h1/h2. The H3K9me3 modification is bound by the HP1 chromo domain. HP1 recruits ADNP via interaction of its chromoshadow domain with the PxVxL motif in the ADNP homeodomain. Finally, ADNP induces transcriptional silencing of major satellite repeats. Further experiments are required to elucidate the exact mechanism of how silencing is mediated.

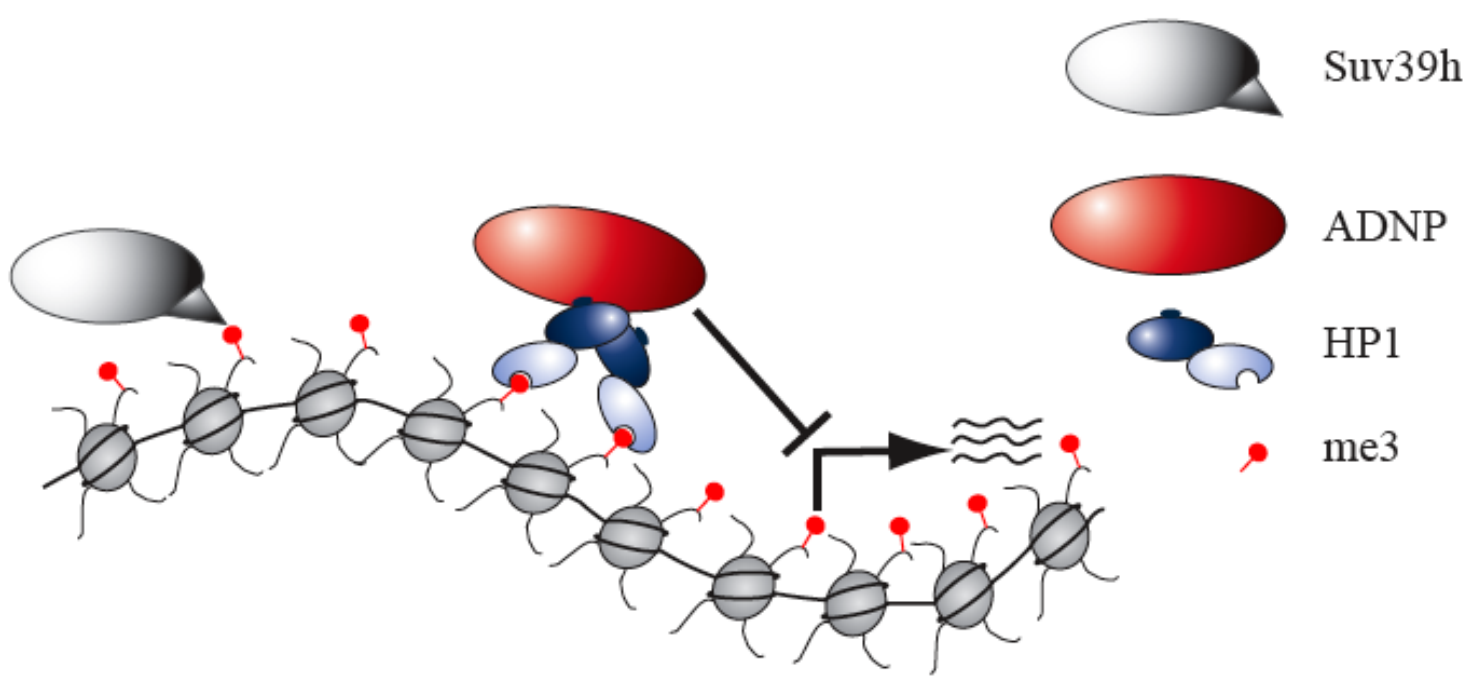

Figure 4-2: Model for ADNP recruitment and function

Suv39h (grey) trimethylates histone H3 at lysine 9 (H3K9me3; small red circle). HP1 via its chromo domain (light blue) to chromatin. HP1 recruits ADNP (red) via binding of the dimeric HP1 chromoshadow domain (dark blue) to a PxVxL motif. Upon recruitment to chromatin, ADNP silences major satellite transcription by an unknown mechanism. 


\section{References}

Aagaard, L., Laible, G., Selenko, P., Schmid, M., Dorn, R., Schotta, G., Kuhfittig, S., Wolf, A., Lebersorger, A., Singh, P.B., et al. (1999). Functional mammalian homologues of the Drosophila PEV-modifier Su(var)3-9 encode centromere-associated proteins which complex with the heterochromatin component M31. Embo J 18, 1923-1938.

Adams-Cioaba, M.A., and Min, J. (2009). Structure and function of histone methylation binding proteins. Biochem Cell Biol 87, 93-105.

Agalioti, T., Chen, G., and Thanos, D. (2002). Deciphering the transcriptional histone acetylation code for a human gene. Cell $111,381-392$.

Agalioti, T., Lomvardas, S., Parekh, B., Yie, J., Maniatis, T., and Thanos, D. (2000). Ordered recruitment of chromatin modifying and general transcription factors to the IFN-beta promoter. Cell 103, 667-678.

Agger, K., Christensen, J., Cloos, P.A., and Helin, K. (2008). The emerging functions of histone demethylases. Curr Opin Genet Dev 18, 159-168.

Ahmad, K., and Henikoff, S. (2002). Epigenetic consequences of nucleosome dynamics. Cell 111, 281284.

Allis, C.D., Bowen, J.K., Abraham, G.N., Glover, C.V., and Gorovsky, M.A. (1980). Proteolytic processing of histone $\mathrm{H3}$ in chromatin: a physiologically regulated event in Tetrahymena micronuclei. Cell 20, 55-64.

Allis, C.D., Jenuwein, T., and Reinberg, D. (2007). Epigenetics. Cold Spring Harbor: Cold Spring Harbor Laboratory Press.

Angelov, D., Vitolo, J.M., Mutskov, V., Dimitrov, S., and Hayes, J.J. (2001). Preferential interaction of the core histone tail domains with linker DNA. Proc Natl Acad Sci U S A 98, 6599-6604.

Arents, G., Burlingame, R.W., Wang, B.C., Love, W.E., and Moudrianakis, E.N. (1991). The nucleosomal core histone octamer at $3.1 \mathrm{~A}$ resolution: a tripartite protein assembly and a left-handed superhelix. Proc Natl Acad Sci U S A 88, 10148-10152.

Ausubel, F.M., Brent, R., Kingston, R.E., Moore, D.D., Seidman, J.G., Smith, J.A., and Struhl, K. (1998). Current Protocols in Molecular Biology (John Wiley \& Sons, Inc.).

Avery, O.T., Macleod, C.M., and McCarty, M. (1944). Studies on the Chemical Nature of the Substance Inducing Transformation of Pneumococcal Types : Induction of Transformation by a Desoxyribonucleic Acid Fraction Isolated from Pneumococcus Type Iii. J Exp Med 79, 137-158.

Bannister, A.J., Zegerman, P., Partridge, J.F., Miska, E.A., Thomas, J.O., Allshire, R.C., and Kouzarides, T. (2001). Selective recognition of methylated lysine 9 on histone H3 by the HP1 chromo domain. Nature 410, 120-124.

Barski, A., Cuddapah, S., Cui, K., Roh, T.Y., Schones, D.E., Wang, Z., Wei, G., Chepelev, I., and Zhao, K. (2007). High-resolution profiling of histone methylations in the human genome. Cell 129, 823-837. 
Bassan, M., Zamostiano, R., Davidson, A., Pinhasov, A., Giladi, E., Perl, O., Bassan, H., Blat, C., Gibney, G., Glazner, G., et al. (1999). Complete sequence of a novel protein containing a femtomolaractivity-dependent neuroprotective peptide. J Neurochem 72, 1283-1293.

Beni-Adani, L., Gozes, I., Cohen, Y., Assaf, Y., Steingart, R.A., Brenneman, D.E., Eizenberg, O., Trembolver, V., and Shohami, E. (2001). A peptide derived from activity-dependent neuroprotective protein (ADNP) ameliorates injury response in closed head injury in mice. J Pharmacol Exp Ther 296, 57-63.

Bernstein, B.E., Mikkelsen, T.S., Xie, X., Kamal, M., Huebert, D.J., Cuff, J., Fry, B., Meissner, A., Wernig, M., Plath, K., et al. (2006). A bivalent chromatin structure marks key developmental genes in embryonic stem cells. Cell $125,315-326$.

Bertin, A., Renouard, M., Pedersen, J.S., Livolant, F., and Durand, D. (2007). H3 and H4 histone tails play a central role in the interactions of recombinant NCPs. Biophys $\mathrm{J}$ 92, 2633-2645.

Bestor, T.H. (2000). The DNA methyltransferases of mammals. Hum Mol Genet 9, 2395-2402.

Bourgo, R.J., Siddiqui, H., Fox, S., Solomon, D., Sansam, C.G., Yaniv, M., Muchardt, C., Metzger, D., Chambon, P., Roberts, C.W., et al. (2009). SWI/SNF deficiency results in aberrant chromatin organization, mitotic failure, and diminished proliferative capacity. Mol Biol Cell 20, 3192-3199.

Brasher, S.V., Smith, B.O., Fogh, R.H., Nietlispach, D., Thiru, A., Nielsen, P.R., Broadhurst, R.W., Ball, L.J., Murzina, N.V., and Laue, E.D. (2000). The structure of mouse HP1 suggests a unique mode of single peptide recognition by the shadow chromo domain dimer. Embo J 19, 1587-1597.

Burkhart, C.A., Kavallaris, M., and Band Horwitz, S. (2001). The role of beta-tubulin isotypes in resistance to antimitotic drugs. Biochim Biophys Acta 1471, 01-9.

Chang, B., Chen, Y., Zhao, Y., and Bruick, R.K. (2007). JMJD6 is a histone arginine demethylase. Science $318,444-447$.

Chen, S., and Charness, M.E. (2008). Ethanol inhibits neuronal differentiation by disrupting activitydependent neuroprotective protein signaling. Proc Natl Acad Sci U S A 105, 19962-19967.

Chuang, L.S., Ian, H.I., Koh, T.W., Ng, H.H., Xu, G., and Li, B.F. (1997). Human DNA-(cytosine-5) methyltransferase-PCNA complex as a target for p21WAF1. Science 277, 1996-2000.

Clements, A., Poux, A.N., Lo, W.S., Pillus, L., Berger, S.L., and Marmorstein, R. (2003). Structural basis for histone and phosphohistone binding by the GCN5 histone acetyltransferase. Mol Cell 12, 461-473.

Collins, R.E., Northrop, J.P., Horton, J.R., Lee, D.Y., Zhang, X., Stallcup, M.R., and Cheng, X. (2008). The ankyrin repeats of G9a and GLP histone methyltransferases are mono- and dimethyllysine binding modules. Nat Struct Mol Biol 15, 245-250.

Cosma, M.P., Tanaka, T., and Nasmyth, K. (1999). Ordered recruitment of transcription and chromatin remodeling factors to a cell cycle- and developmentally regulated promoter. Cell 97, 299311.

Cowieson, N.P., Partridge, J.F., Allshire, R.C., and McLaughlin, P.J. (2000). Dimerisation of a chromo shadow domain and distinctions from the chromodomain as revealed by structural analysis. Curr Biol, 517-525. 
Daujat, S., Zeissler, U., Waldmann, T., Happel, N., and Schneider, R. (2005). HP1 binds specifically to Lys26-methylated histone H1.4, whereas simultaneous Ser27 phosphorylation blocks HP1 binding. J Biol Chem, 38090-38095.

Davey, C.A., Sargent, D.F., Luger, K., Maeder, A.W., and Richmond, T.J. (2002). Solvent mediated interactions in the structure of the nucleosome core particle at 1.9 a resolution. J Mol Biol 319, 10971113.

Delattre, M., Spierer, A., Tonka, C.H., and Spierer, P. (2000). The genomic silencing of positioneffect variegation in Drosophila melanogaster: interaction between the heterochromatin-associated proteins Su(var)3-7 and HP1. J Cell Sci, 4253-4261.

Dhalluin, C., Carlson, J.E., Zeng, L., He, C., Aggarwal, A.K., and Zhou, M.M. (1999). Structure and ligand of a histone acetyltransferase bromodomain. Nature 399, 491-496.

Divinski, I., Holtser-Cochav, M., Vulih-Schultzman, I., Steingart, R.A., and Gozes, I. (2006). Peptide neuroprotection through specific interaction with brain tubulin. J Neurochem 98, 973-984.

Divinski, I., Mittelman, L., and Gozes, I. (2004). A femtomolar acting octapeptide interacts with tubulin and protects astrocytes against zinc intoxication. $J$ Biol Chem 279, 28531-28538.

Dormann, H. (2009). REGULATION OF HETEROCHROMATIN PROTEIN 1 BY PHOSPHORYLATION OF HISTONE H3 AND THE HP1 HINGE DOMAIN (New York, The Rockefeller University).

Duncan, E.M., Muratore-Schroeder, T.L., Cook, R.G., Garcia, B.A., Shabanowitz, J., Hunt, D.F., and Allis, C.D. (2008). Cathepsin L proteolytically processes histone H3 during mouse embryonic stem cell differentiation. Cell 135, 284-294.

Eroglu, B., Wang, G., Tu, N., Sun, X., and Mivechi, N.F. (2006). Critical role of Brg1 member of the SWI/SNF chromatin remodeling complex during neurogenesis and neural crest induction in zebrafish. Dev Dyn 235, 2722-2735.

Felsenfeld, G., and Groudine, M. (2003). Controlling the double helix. Nature 421, 448-453.

Fischle, W. (2005). In nucleo enzymatic assays for the identification and characterization of histone modifying activities. Methods 36, 362-367.

Fischle, W., Franz, H., Jacobs, S.A., Allis, C.D., and Khorasanizadeh, S. (2008). Specificity of the chromodomain Y chromosome family of chromodomains for lysine-methylated ARK(S/T) motifs. J Biol Chem 283, 19626-19635.

Fischle, W., Tseng, B.S., Dormann, H.L., Ueberheide, B.M., Garcia, B.A., Shabanowitz, J., Hunt, D.F., Funabiki, H., and Allis, C.D. (2005). Regulation of HP1-chromatin binding by histone H3 methylation and phosphorylation. Nature 438, 1116-1122.

Fischle, W., Wang, Y., and Allis, C.D. (2003a). Binary switches and modification cassettes in histone biology and beyond. Nature $425,475-479$.

Fischle, W., Wang, Y., and Allis, C.D. (2003b). Histone and chromatin cross-talk. Curr Opin Cell Biol 15, 172-183.

Fischle, W., Wang, Y., Jacobs, S.A., Kim, Y., Allis, C.D., and Khorasanizadeh, S. (2003c). Molecular basis for the discrimination of repressive methyl-lysine marks in histone H3 by Polycomb and HP1 chromodomains. Genes Dev 17, 1870-1881. 
Francis, N.J., Kingston, R.E., and Woodcock, C.L. (2004). Chromatin compaction by a polycomb group protein complex. Science 306, 1574-1577.

Franz, H., Mosch, K., Soeroes, S., Urlaub, H., and Fischle, W. (2009). Multimerization and H3K9me3 binding is required for CDYL1b heterochromatin association. J Biol Chem 5,5 .

Furman, S., Steingart, R.A., Mandel, S., Hauser, J.M., Brenneman, D.E., and Gozes, I. (2004). Subcellular localization and secretion of activity-dependent neuroprotective protein in astrocytes. Neuron Glia Biol 1, 193-199.

Gaudin, V., Libault, M., Pouteau, S., Juul, T., Zhao, G., Lefebvre, D., and Grandjean, O. (2001). Mutations in LIKE HETEROCHROMATIN PROTEIN 1 affect flowering time and plant architecture in Arabidopsis. Development 128, 4847-4858.

Gozes, I., and Brenneman, D.E. (2000). A new concept in the pharmacology of neuroprotection. J Mol Neurosci 14, 61-68.

Gozes, I., Morimoto, B.H., Tiong, J., Fox, A., Sutherland, K., Dangoor, D., Holser-Cochav, M., Vered, K., Newton, P., Aisen, P.S., et al. (2005). NAP: research and development of a peptide derived from activity-dependent neuroprotective protein (ADNP). CNS Drug Rev 11, 353-368.

Gozes, I., Zamostiano, R., Pinhasov, A., Bassan, M., Giladi, E., Steingart, R.A., and Brenneman, D.E. (2000). A novel VIP responsive gene. Activity dependent neuroprotective protein. Ann N Y Acad Sci 921, 115-118.

Grewal, S.I., and Elgin, S.C. (2007). Transcription and RNA interference in the formation of heterochromatin. Nature 447, 399-406.

Grunstein, M. (1997). Molecular model for telomeric heterochromatin in yeast. Curr Opin Cell Biol 9, 383-387.

Guccione, E., Bassi, C., Casadio, F., Martinato, F., Cesaroni, M., Schuchlautz, H., Luscher, B., and Amati, B. (2007). Methylation of histone H3R2 by PRMT6 and H3K4 by an MLL complex are mutually exclusive. Nature $449,933-937$.

Hansen, J.C. (2002). Conformational dynamics of the chromatin fiber in solution: determinants, mechanisms, and functions. Annu Rev Biophys Biomol Struct 31, 361-392.

Hansen, J.C. (2006). Linking genome structure and function through specific histone acetylation. ACS Chem Biol 1, 69-72.

Hansen, J.C., Tse, C., and Wolffe, A.P. (1998). Structure and function of the core histone N-termini: more than meets the eye. Biochemistry 37, 17637-17641.

Harnicarova Horakova, A., Galiova, G., Legartova, S., Kozubek, S., Matula, P., and Bartova, E. (2009). Chromocentre integrity and epigenetic marks. J Struct Biol 169, 124-133.

Hassa, P.O., Haenni, S.S., Elser, M., and Hottiger, M.O. (2006). Nuclear ADP-ribosylation reactions in mammalian cells: where are we today and where are we going? Microbiol Mol Biol Rev 70, 789829.

Heintzman, N.D., Hon, G.C., Hawkins, R.D., Kheradpour, P., Stark, A., Harp, L.F., Ye, Z., Lee, L.K., Stuart, R.K., Ching, C.W., et al. (2009). Histone modifications at human enhancers reflect global celltype-specific gene expression. Nature 459, 108-112. 
Heitz, E. (1928). Das Heterochromatin der Moose. Jahrb Wiss Botainik 69, 762-818.

Hill, D.A., Chiosea, S., Jamaluddin, S., Roy, K., Fischer, A.H., Boyd, D.D., Nickerson, J.A., and Imbalzano, A.N. (2004). Inducible changes in cell size and attachment area due to expression of a mutant SWI/SNF chromatin remodeling enzyme. J Cell Sci 117, 5847-5854.

Hofmann, K. (2009). Ubiquitin-binding domains and their role in the DNA damage response. DNA Repair (Amst) 8, 544-556.

Holbert, M.A., and Marmorstein, R. (2005). Structure and activity of enzymes that remove histone modifications. Curr Opin Struct Biol 15, 673-680.

Huynh, V.A., Robinson, P.J., and Rhodes, D. (2005). A method for the in vitro reconstitution of a defined "30 nm" chromatin fibre containing stoichiometric amounts of the linker histone. J Mol Biol $345,957-968$.

Iberg, A.N., Espejo, A., Cheng, D., Kim, D., Michaud-Levesque, J., Richard, S., and Bedford, M.T. (2008). Arginine methylation of the histone $\mathrm{H3}$ tail impedes effector binding. J Biol Chem 283, 30063010 .

Ishizuka, T., and Lazar, M.A. (2003). The N-CoR/histone deacetylase 3 complex is required for repression by thyroid hormone receptor. Mol Cell Biol 23, 5122-5131.

Jacobs, S.A., and Khorasanizadeh, S. (2002). Structure of HP1 chromodomain bound to a lysine 9methylated histone $\mathrm{H3}$ tail. Science 295, 2080-2083.

Jacobs, S.A., Taverna, S.D., Zhang, Y., Briggs, S.D., Li, J., Eissenberg, J.C., Allis, C.D., and Khorasanizadeh, S. (2001). Specificity of the HP1 chromo domain for the methylated N-terminus of histone H3. EMBO J 20, 5232-5241.

Jenuwein, T., and Allis, C.D. (2001). Translating the histone code. Science 293, 1074-1080.

Johansen, K.M., and Johansen, J. (2006). Regulation of chromatin structure by histone H3S10 phosphorylation. Chromosome Res 14, 393-404.

Johnson, A., Li, G., Sikorski, T.W., Buratowski, S., Woodcock, C.L., and Moazed, D. (2009). Reconstitution of heterochromatin-dependent transcriptional gene silencing. Mol Cell 35, 769-781.

Kalakonda, N., Fischle, W., Boccuni, P., Gurvich, N., Hoya-Arias, R., Zhao, X., Miyata, Y., Macgrogan, D., Zhang, J., Sims, J.K., et al. (2008). Histone H4 lysine 20 monomethylation promotes transcriptional repression by L3MBTL1. Oncogene 27, 4293-4304.

Karagianni, P., Amazit, L., Qin, J., and Wong, J. (2008). ICBP90, a novel methyl K9 H3 binding protein linking protein ubiquitination with heterochromatin formation. Mol Cell Biol 28, $705-717$.

Katsetos, C.D., Legido, A., Perentes, E., and Mork, S.J. (2003). Class III beta-tubulin isotype: a key cytoskeletal protein at the crossroads of developmental neurobiology and tumor neuropathology. $J$ Child Neurol 18, 851-866; discussion 867.

Koester-Eiserfunke, N. (2010). Characterization of Lin-61 methyl mark binding and its function in C. elegans vulva development. In Group of Chromatin Biochemistry (Goettingen, Max Planck Institute for Biophysical Chemistry). 
Koonin, E.V., Zhou, S., and Lucchesi, J.C. (1995). The chromo superfamily: new members, duplication of the chromo domain and possible role in delivering transcription regulators to chromatin. Nucleic Acids Res 23, 4229-4233.

Kornberg, R.D. (1974). Chromatin structure: a repeating unit of histones and DNA. Science 184, 868871.

Kouzarides, T. (2007). Chromatin modifications and their function. Cell 128, 693-705.

L. Vandel, E.N., O. Vaute, R. Ferreira, S. Ait-Si-Ali and D. Trouche (2001). Transcriptional repression by the retinoblastoma protein through the recruitment of a histone methyltransferase. Mol Cell Biol 21, 6484-6494.

Lachner, M., O'Carroll, D., Rea, S., Mechtler, K., and Jenuwein, T. (2001). Methylation of histone H3 lysine 9 creates a binding site for HP1 proteins. Nature 410, 116-120.

Laemmli, U.K. (1970). Cleavage of structural proteins during the assembly of the head of bacteriophage T4. Natue $227,680-685$.

Lander, E.S., Linton, L.M., Birren, B., Nusbaum, C., Zody, M.C., Baldwin, J., Devon, K., Dewar, K., Doyle, M., FitzHugh, W., et al. (2001). Initial sequencing and analysis of the human genome. Nature $409,860-921$.

Lee, B.M., and Mahadevan, L.C. (2009). Stability of histone modifications across mammalian genomes: implications for 'epigenetic' marking. J Cell Biochem 108, 22-34.

Lehnertz, B., Ueda, Y., Derijck, A.A., Braunschweig, U., Perez-Burgos, L., Kubicek, S., Chen, T., Li, E., Jenuwein, T., and Peters, A.H. (2003). Suv39h-mediated histone H3 lysine 9 methylation directs DNA methylation to major satellite repeats at pericentric heterochromatin. Curr Biol 13, 1192-1200.

Linder, B., Gerlach, N., and Jackle, H. (2001). The Drosophila homolog of the human AF10 is an HP1-interacting suppressor of position effect variegation. EMBO Rep 2, 211-216.

Lowary, P.T., and Widom, J. (1998). New DNA sequence rules for high affinity binding to histone octamer and sequence-directed nucleosome positioning. J Mol Biol 276, 19-42.

Luger, K., Mader, A.W., Richmond, R.K., Sargent, D.F., and Richmond, T.J. (1997). Crystal structure of the nucleosome core particle at 2.8 A resolution. Nature $389,251-260$.

Luger, K., Rechsteiner, T.J., and Richmond, T.J. (1999). Preparation of nucleosome core particle from recombinant histones. Methods Enzymol 304, 3-19.

Maison, C., Bailly, D., Peters, A.H., Quivy, J.P., Roche, D., Taddei, A., Lachner, M., Jenuwein, T., and Almouzni, G. (2002). Higher-order structure in pericentric heterochromatin involves a distinct pattern of histone modification and an RNA component. Nat Genet 30, 329-334.

Mandel, S., and Gozes, I. (2007). Activity-dependent neuroprotective protein constitutes a novel element in the SWI/SNF chromatin remodeling complex. J Biol Chem 282, 34448-34456.

Mandel, S., Rechavi, G., and Gozes, I. (2007). Activity-dependent neuroprotective protein (ADNP) differentially interacts with chromatin to regulate genes essential for embryogenesis. Dev Biol 303 , 814-824.

Mangenot, S., Leforestier, A., Vachette, P., Durand, D., and Livolant, F. (2002). Salt-induced conformation and interaction changes of nucleosome core particles. Biophys $\mathbf{J}$ 82, 345-356. 
Martens, J.H., O'Sullivan, R.J., Braunschweig, U., Opravil, S., Radolf, M., Steinlein, P., and Jenuwein, T. (2005). The profile of repeat-associated histone lysine methylation states in the mouse epigenome. EMBO J 24, 800-812.

Melcher, M., Schmid, M., Aagaard, L., Selenko, P., Laible, G., and Jenuwein, T. (2000). Structurefunction analysis of SUV39H1 reveals a dominant role in heterochromatin organization, chromosome segregation, and mitotic progression. Mol Cell Biol 20, 3728-3741.

Metzger, E., Wissmann, M., Yin, N., Muller, J.M., Schneider, R., Peters, A.H., Gunther, T., Buettner, R., and Schule, R. (2005). LSD1 demethylates repressive histone marks to promote androgenreceptor-dependent transcription. Nature 437, 436-439.

Mikkelsen, T.S., Ku, M., Jaffe, D.B., Issac, B., Lieberman, E., Giannoukos, G., Alvarez, P., Brockman, W., Kim, T.K., Koche, R.P., et al. (2007). Genome-wide maps of chromatin state in pluripotent and lineage-committed cells. Nature 448, 553-560.

Mujtaba, S., Zeng, L., and Zhou, M.M. (2007). Structure and acetyl-lysine recognition of the bromodomain. Oncogene 26, 5521-5527.

Muller, H.J., and Altenburg, E. (1930). The Frequency of Translocations Produced by X-Rays in Drosophila. Genetics 15, 283-311.

Nathan, D., Ingvarsdottir, K., Sterner, D.E., Bylebyl, G.R., Dokmanovic, M., Dorsey, J.A., Whelan, K.A., Krsmanovic, M., Lane, W.S., Meluh, P.B., et al. (2006). Histone sumoylation is a negative regulator in Saccharomyces cerevisiae and shows dynamic interplay with positive-acting histone modifications. Genes Dev 20, 966-976.

Nelson, C.J., Santos-Rosa, H., and Kouzarides, T. (2006). Proline isomerization of histone $\mathrm{H3}$ regulates lysine methylation and gene expression. Cell 126, 905-916.

Nielsen, A.L., Oulad-Abdelghani, M., Ortiz, J.A., Remboutsika, E., Chambon, P., and Losson, R. (2001a). Heterochromatin formation in mammalian cells: interaction between histones and HP1 proteins. Mol Cell 7, 729-739.

Nielsen, P.R., Nietlispach, D., Mott, H.R., Callaghan, J.M., Bannister, A.J., Kouzarides, T., Murzin, A.G., Murzina, N.V., and Laue, E.D. (2002). Structure of the HP1 chromodomain bound to histone H3 methylated at lysine 9. Nature 416, 103-107.

Nielsen, S.J., Schneider, R., Bauer, U.M., Bannister, A.J., Morrison, A., O'Carroll, D., Firestein, R., Cleary, M., Jenuwein, T., Herrera, R.E., et al. (2001b). Rb targets histone H3 methylation and HP1 to promoters. Nature 412, 561-565.

Nowak, S.J., and Corces, V.G. (2004). Phosphorylation of histone H3: a balancing act between chromosome condensation and transcriptional activation. Trends Genet 20, 214-220.

Nozawa, N.S., K., N., H.T., M., O., I., T., H., N., N., H., K., and C., O. (2010). Human POGZ modulates dissociation of HP1alpha from mitotic chromosome arms through Aurora B activation. Nat Cell Biol.

Olins, A.L., and Olins, D.E. (1974). Spheroid chromatin units (v bodies). Science 183, 330-332.

Ong, S.E., and Mann, M. (2005). Mass spectrometry-based proteomics turns quantitative. Nat Chem Biol 1, 252-262. 
Ong, S.E., and Mann, M. (2006). A practical recipe for stable isotope labeling by amino acids in cell culture (SILAC). Nat Protoc $1,2650-2660$.

Peters, A.H., Kubicek, S., Mechtler, K., O'Sullivan, R.J., Derijck, A.A., Perez-Burgos, L., KohImaier, A., Opravil, S., Tachibana, M., Shinkai, Y., et al. (2003). Partitioning and plasticity of repressive histone methylation states in mammalian chromatin. Mol Cell 12, 1577-1589.

Peters, A.H., O'Carroll, D., Scherthan, H., Mechtler, K., Sauer, S., Schofer, C., Weipoltshammer, K., Pagani, M., Lachner, M., Kohlmaier, A., et al. (2001). Loss of the Suv39h histone methyltransferases impairs mammalian heterochromatin and genome stability. Cell 107, 323-337.

Peterson, C.L., and Laniel, M.A. (2004). Histones and histone modifications. . Curr Biol 14, R546551.

Pinhasov, A., Mandel, S., Torchinsky, A., Giladi, E., Pittel, Z., Goldsweig, A.M., Servoss, S.J., Brenneman, D.E., and Gozes, I. (2003). Activity-dependent neuroprotective protein: a novel gene essential for brain formation. Brain Res Dev Brain Res 144, 83-90.

Platero J.S., H.T., and Eissenberg J.C. (1995). Functional analysis of the chromo domain of HP1. EMBO J, 3977-3986.

Platero, J.S., Hartnett, T., and Eissenberg, J.C. (1995). Functional analysis of the chromo domain of HP1. EMBO J, 3977-3986.

Prigent, C., and Dimitrov, S. (2003). Phosphorylation of serine 10 in histone H3, what for? J Cell Sci 116, 3677-3685.

Przewloca, M.R., and Glower, M.G. (2009). The Kinetochore and the Centromere: A Working Long Distance Relationship. Annu Rev Genet 43, 439-465.

Qian, C., and Zhou, M.M. (2006). SET domain protein lysine methyltransferases: Structure, specificity and catalysis. Cell Mol Life Sci 63, 2755-2763.

Qu, J.H., Cheng, J., Zhang, L.X., Zhong, Y.W., Liu, Y., Wang, L., and Dai, J.Z. (2005). [Screening of binding proteins to interferon-alpha promoter DNA by phage display technique]. Zhonghua Gan Zang Bing Za Zhi 13, 520-523.

Quintana, F.J., Zaltzman, R., Fernandez-Montesinos, R., Herrera, J.L., Gozes, I., Cohen, I.R., and Pozo, D. (2006). NAP, a peptide derived from the activity-dependent neuroprotective protein, modulates macrophage function. Ann N Y Acad Sci 1070, 500-506.

Reik, W., Dean, W., and Walter, J. (2001). Epigenetic reprogramming in mammalian development. Science 293, 1089-1093.

Reuter, G., Gausz, J., Gyurkovics, H., Friede, B., Bang, R., Spierer, A., Hall, L.M., and Spierer, P. (1987). Modifiers of position-effect variegation in the region from $86 \mathrm{C}$ to $88 \mathrm{~B}$ of the Drosophila melanogaster third chromosome. Mol Gen Genet 210, 429-436.

Rosenfeld, J.A., Wang, Z., Schones, D.E., Zhao, K., DeSalle, R., and Zhang, M.Q. (2009). Determination of enriched histone modifications in non-genic portions of the human genome. BMC Genomics 10, 143.

Rosson, G.B., Bartlett, C., Reed, W., and Weissman, B.E. (2005). BRG1 loss in MiaPaCa2 cells induces an altered cellular morphology and disruption in the organization of the actin cytoskeleton. $J$ Cell Physiol 205, 286-294. 
Rountree, M.R., Bachman, K.E., and Baylin, S.B. (2000). DNMT1 binds HDAC2 and a new corepressor, DMAP1, to form a complex at replication foci. Nat Genet 25, 269-277.

Sambrook, J., and Russel, D. (2001). Molecular Cloning. A laboratory Manual, 3rd edn (Cold Spring Harbor, New York, Cold Spring Harbor Laboratory Press).

Sampath, S.C., Marazzi, I., Yap, K.L., Sampath, S.C., Krutchinsky, A.N., Mecklenbrauker, I., Viale, A., Rudensky, E., Zhou, M.M., Chait, B.T., et al. (2007). Methylation of a histone mimic within the histone methyltransferase G9a regulates protein complex assembly. Mol Cell 27, 596-608.

Sari, Y., and Gozes, I. (2006). Brain deficits associated with fetal alcohol exposure may be protected, in part, by peptides derived from activity-dependent neurotrophic factor and activity-dependent neuroprotective protein. Brain Res Rev 52, 107-118.

Schotta, G., Lachner, M., Sarma, K., Ebert, A., Sengupta, R., Reuter, G., Reinberg, D., and Jenuwein, T. (2004). A silencing pathway to induce H3-K9 and H4-K20 trimethylation at constitutive heterochromatin. Genes Dev 18, 1251-1262.

Schultz, D.C., Ayyanathan, K., Negorev, D., Maul, G.G., and Rauscher, F.J., 3rd (2002). SETDB1: a novel KAP-1-associated histone H3, lysine 9-specific methyltransferase that contributes to HP1mediated silencing of euchromatic genes by KRAB zinc-finger proteins. Genes Dev 16, 919-932.

Seet, B.T., Dikic, I., Zhou, M.M., and Pawson, T. (2006). Reading protein modifications with interaction domains. Nat Rev Mol Cell Biol 7, 473-483.

Selmi, C., Lleo, A., Zuin, M., Podda, M., Rossaro, L., and Gershwin, M.E. (2006). Interferon alpha and its contribution to autoimmunity. Curr Opin Investig Drugs 7, 451-456.

Seo, S., Richardson, G.A., and Kroll, K.L. (2005). The SWI/SNF chromatin remodeling protein Brg1 is required for vertebrate neurogenesis and mediates transactivation of Ngn and NeuroD. Development 132, 105-115.

Sharma, K., Weber, C., Bairlein, M., Greff, Z., Kéri, G., Cox, J., Olsen, J.V., and Daub, H. (2009). Proteomics strategy for quantitative protein interaction profiling in cell extracts. Natue Methods 6 , 741-744.

Shevchenko, A., Wilm, M., Vorm, O., and Mann, M. (1996). Mass spectrometric sequencing of proteins silver-stained polyacrylamide gels. Anal Chem 68, 850-858.

Shogren-Knaak, M., Ishii, H., Sun, J.M., Pazin, M.J., Davie, J.R., and Peterson, C.L. (2006). Histone H4-K16 acetylation controls chromatin structure and protein interactions. Science 311, 844-847.

Shogren-Knaak, M.A., and Peterson, C.L. (2004). Creating designer histones by native chemical ligation. Methods Enzymol 375, 62-76.

Sigalov, E., Fridkin, M., Brenneman, D.E., and Gozes, I. (2000). VIP-Related protection against lodoacetate toxicity in pheochromocytoma (PC12) cells: a model for ischemic/hypoxic injury. J Mol Neurosci 15, 147-154.

Simone, C. (2006). SWI/SNF: the crossroads where extracellular signaling pathways meet chromatin. J Cell Physiol 207, 309-314.

Smothers, J.F., and Henikoff, S. (2000). The HP1 chromo shadow domain binds a consensus peptide pentamer. Curr Biol, 27-30. 
Soeroes, S. (2010). H3K9me3 dependent HP1 chromatin association and its consequences for chromatin packaging. In Group of Chromatin Biochemistry (Goettingen, Max Planck Institute for Biophysical Chemistry).

Steingart, R.A., and Gozes, I. (2006). Recombinant activity-dependent neuroprotective protein protects cells against oxidative stress. Mol Cell Endocrinol 252, 148-153.

Sterner, D.E., and Berger, S.L. (2000). Acetylation of histones and transcription-related factors. Microbiol Mol Biol Rev 64, 435-459.

Stewart, M.D., Li, J., and Wong, J. (2005). Relationship between histone H3 lysine 9 methylation, transcription repression, and heterochromatin protein 1 recruitment. Mol Cell Biol 25, 2525-2538.

Strahl, B.D., and Allis, C.D. (2000). The language of covalent histone modifications. Nature 403, 4145.

Struhl, K. (1998). Histone acetylation and transcriptional regulatory mechanisms. Genes Dev 12, 599606.

Taverna, S.D., Li, H., Ruthenburg, A.J., Allis, C.D., and Patel, D.J. (2007). How chromatin-binding modules interpret histone modifications: lessons from professional pocket pickers. Nat Struct Mol Biol 14, 1025-1040.

Thastrom, A., Lowary, P.T., Widlund, H.R., Cao, H., Kubista, M., and Widom, J. (1999). Sequence motifs and free energies of selected natural and non-natural nucleosome positioning DNA sequences. J Mol Biol 288, 213-229.

Thiru, A., Nietlispach, D., Mott, H.R., Okuwaki, M., Lyon, D., Nielsen, P.R., Hirshberg, M., Verreault, A., Murzina, N.V., and Laue, E.D. (2004). Structural basis of HP1/PXVXL motif peptide interactions and HP1 localisation to heterochromatin. Embo J 23, 489-499.

Thomas, J.O. (1999). Histone H1: location and role. Curr Opin Cell Biol 11, 312-317.

van Attikum, H., and Gasser, S.M. (2009). Crosstalk between histone modifications during the DNA damage response. Trends Cell Biol 19, 207-217.

Vassallo, M.F., and Tanese, N. (2002). Isoform-specific interaction of HP1 with human TAFII130. Proc Natl Acad Sci U S A 99, 5919-5924.

Vermeulen, M., Mulder, K.W., Denissov, S., Pijnappel, W.W., van Schaik, F.M., Varier, R.A., Baltissen, M.P., Stunnenberg, H.G., Mann, M., and Timmers, H.T. (2007). Selective anchoring of TFIID to nucleosomes by trimethylation of histone $\mathrm{H3}$ lysine 4. Cell 131, 58-69.

Wang, H., An, W., Cao, R., Xia, L., Erdjument-Bromage, H., Chatton, B., Tempst, P., Roeder, R.G., and Zhang, Y. (2003). mAM facilitates conversion by ESET of dimethyl to trimethyl lysine 9 of histone $\mathrm{H3}$ to cause transcriptional repression. Mol Cell 12, 475-487.

Wang, Z., Schones, D.E., and Zhao, K. (2009). Characterization of human epigenomes. Curr Opin Genet Dev 19, 127-134.

Weake, V.M., and Workman, J.L. (2008). Histone ubiquitination: triggering gene activity. Mol Cell 29, 653-663.

Weiler, K.S., and Wakimoto, B.T. (1995). Heterochromatin and gene expression in Drosophila. Annu Rev Genet 29, 577-605. 
Wustmann, G., Szidonya, J., Taubert, H., and Reuter, G. (1989). The genetics of position-effect variegation modifying loci in Drosophila melanogaster. Mol Gen Genet 217, 520-527.

Wysocka, J., Swigut, T., Milne, T.A., Dou, Y., Zhang, X., Burlingame, A.L., Roeder, R.G., Brivanlou, A.H., and Allis, C.D. (2005). WDR5 associates with histone $\mathrm{H3}$ methylated at $\mathrm{K} 4$ and is essential for H3 K4 methylation and vertebrate development. Cell 121, 859-872.

Zamostiano, R., Pinhasov, A., Gelber, E., Steingart, R.A., Seroussi, E., Giladi, E., Bassan, M., Wollman, Y., Eyre, H.J., Mulley, J.C., et al. (2001). Cloning and characterization of the human activity-dependent neuroprotective protein. J Biol Chem 276, 708-714.

Zeng, L., and Zhou, M.M. (2002). Bromodomain: an acetyl-lysine binding domain. FEBS Lett 513, 124-128.

Zhang, Y., and Reinberg, D. (2001). Transcription regulation by histone methylation: interplay between different covalent modifications of the core histone tails. Genes Dev 15, 2343-2360.

Zhao, T., Heyduk, T., Allis, C.D., and Eissenberg, J.C. (2000). Heterochromatin protein 1 binds to nucleosomes and DNA in vitro. J Biol Chem 275, 28332-28338. 


\section{Curriculum vitae}

Personal Details

Name: $\quad$ Kerstin Mosch

Date of birth: $\quad$ August 14, 1970

Place of birth: $\quad$ Dresden, Germany

Nationality: German

\section{Work Experience:}

10.2005-12.2005 Biotechnological Centre of the TU Dresden, Proteomics group /Genomics group; Student assistant

08.2004-11.2004 Biotechnological Centre of the TU Dresden, Proteomics group; Student assistant

05.2004-08.2004 Biotechnological Centre of the TU Dresden, Bioinformatics group; Student assistant

08.2002 - 09.2002 Institut of Polymer Research, Dresden; Internship

$08.2001-09.2001$ TU Dresden, Institut of Zoology; Student assistant

02.1994 - 04.2000 Dresdner Bank AG in Dresden; Bank employee

education

since $07 / 2006$

GGNB "Molecular Biology of Microbial, Animal and Plant Cells", Georg-August-University Göttingen 
PhD studies, Thesis: "Identification and characterization of ADNP as a novel heterochromatin component"

03.2005-09.2005 Biotechnological Centre of the TU Dresden

Master studies, Thesis: "Generation of recombinant adenoviruses for functional analysis of osteoclasts“

04.2003 - 08.2003 TU Dresden, Institut of Zoology

Bachelor studies, Thesis: "Nachweis von Varianten der Östrogenrezeptoren $\alpha$ und $\beta$ in Gebärmutterkarzinomzelllinien“

09.1997-07.2000 Abendgymnasium Dresden; Abitur

\section{Stipends}

2006 - $2008 \quad$ Max-Planck-Institute stipend

\section{Publications}

Franz, H., Sörös, S., Mosch, K., Urlaub, H., and W. Fischle, Multimerization and H3K9me3 binding is required for CDYL1b heterochromatin association. $J$ Biol Chem, 2009; Dec 11;284(50):35049-59

Heckel T, Czupalla C, Expirto Santo AI, Anitei M, Arantzazu Sanchez-Fernandez M, Mosch K, Krause E and Hoflack B., Src-dependent repression of ARF6 is required to maintain podosome-rich sealing zones in bone-digesting osteoclasts. Proc Natl Acad Sci U S A, 2009 Feb 3; 106(5):1451-6

Zhang K, Mosch K, Fischle W and Grewal SI., Roles of the Clr4 methyltransferase complex in nucleation, spreading and maintenance of heterochromatin. Nat Struct Mol Biol. 2008 Apr; 15(4):381-8. 